University of Louisville

ThinkIR: The University of Louisville's Institutional Repository

Electronic Theses and Dissertations

$8-2020$

\title{
Epithelial responses to Porphyromonas gingivalis in the community context.
}

Zackary Ray Fitzsimonds

University of Louisville

Follow this and additional works at: https://ir.library.louisville.edu/etd

Part of the Oral Biology and Oral Pathology Commons

\section{Recommended Citation}

Fitzsimonds, Zackary Ray, "Epithelial responses to Porphyromonas gingivalis in the community context." (2020). Electronic Theses and Dissertations. Paper 3508.

https://doi.org/10.18297/etd/3508

This Doctoral Dissertation is brought to you for free and open access by ThinkIR: The University of Louisville's Institutional Repository. It has been accepted for inclusion in Electronic Theses and Dissertations by an authorized administrator of ThinkIR: The University of Louisville's Institutional Repository. This title appears here courtesy of the author, who has retained all other copyrights. For more information, please contact thinkir@louisville.edu. 


\title{
EPITHELIAL RESPONSES TO PORPHYROMONAS GINGIVALIS IN THE COMMUNITY
} CONTEXT

\author{
By \\ Zackary Ray Fitzsimonds
}

\author{
A Dissertation \\ Submitted to the Faculty of the \\ Graduate School of the University of Louisville \\ In Partial Fulfillment of the Requirements \\ For the Degree of
}

Doctor of Philosophy in Interdisciplinary Studies

Interdisciplinary and Graduate Studies

University of Louisville

Louisville, KY

August 2020 



\title{
EPITHELIAL RESPONSES TO PORPHYROMONAS GINGIVALIS IN THE COMMUNITY
} CONTEXT

\author{
By \\ Zackary Ray Fitzsimonds \\ A Dissertation Approved on \\ July 14, 2020 \\ By the following Dissertation Committee \\ Dr. Richard Lamont, Chair \\ Dr. Carolyn Klinge \\ Dr. Thomas Mitchell \\ Dr. Lisa Sandell \\ Dr. Brian Shumway
}




\section{ACKNOWLEDGEMENTS}

I would like to thank my mentor, Dr. Richard Lamont, for his endless support and patience throughout the Ph.D. phase of the D.M.D./Ph.D. program. I'd also like to thank Dr. Brian Shumway for his role in my training as a clinical mentor, which was extremely beneficial in developing the foundation for my understanding of translational research. I also appreciate Dr. Lisa Sandell for the countless hours spent discussing my research, and for her training on several techniques required to complete this project. I'm also grateful for Dr. Thomas Mitchell and Dr. Carolyn Klinge for their help in refining and improving the scientific rigor of this project as members of my dissertation committee. I'd also like to thank my future wife Mallory for her continued support and patience. Without her I could not have completed this dissertation. Lastly, I would like to thank my family, especially my grandparents, for their unconditional love and encouragement, which made the completion of this program possible. 


\section{ABSTRACT \\ EPITHELIAL RESPONSES TO PORPHYROMONAS GINGIVALIS IN THE COMMUNITY CONTEXT \\ Zackary Ray Fitzsimonds}

July 14,2020

Porphyromonas gingivalis, a keystone pathogen for periodontal disease, initiates a complex molecular dialogue with gingival epithelial cells, leading ultimately to disruption of host signaling pathways. Dysbiotic host responses are also thought to contribute to the initiation and progression of oral squamous cell carcinomas. Recently, the homeostatic commensal Streptococcus gordonii has been shown to antagonize $P$. gingivalis-induced epithelial cell signaling events in host cells, such as proliferation and migration. The aim of this study was to characterize pathways that $P$. gingivalis targets to disturb host signaling, with a specific focus on pathways that S. gordonii can restore to homeostatic levels in the presence of $P$. gingivalis. RNAseq analysis of gingival epithelial cells challenged with $P$. gingivalis revealed a transcriptional pattern reflecting activation of Notch signaling. Signaling is activated by proteolytic cleavage of Notch and Jag1 by gingipain proteases and leads to upregulation of Olfactomedin 4 (OLFM4). This activation is antagonized by $S$. gordonii through the secretion of hydrogen peroxide, which inactivates the gingipains, preventing proteolytic cleavage of Notch1 and Jag1, ultimately blocking OLFM4 upregulation. OLFM4 is required for $P$. gingivalis-induced epithelial cell 
migration. RNAseq analysis identified an OLFM4-independent immune signature that included increased expression of CXCL8, CXCL10, and CXCL11 in epithelial cells transfected with siRNA and then challenged with $P$. gingivalis. In addition, a tissue culture model revealed that OLFM4 was required for $P$. gingivalis-induced epithelial cell migration. OLFM4 therefore represents a new axis by which $P$. gingivalis disturbs homeostasis at the mucosal barrier, as well as an additional mechanism for ameliorating this disruption by S. gordonii. This study provides insight into the complex molecular dialogue at the mucosal-microbe interface, and as a result expands on the need for new therapeutic approaches to prevent and identify those at high-risk for oral squamous cell carcinoma. 


\section{TABLE OF CONTENTS}

\section{Page}

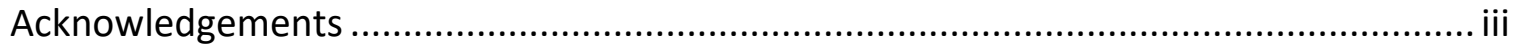

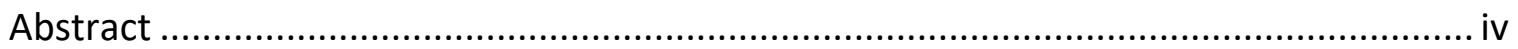

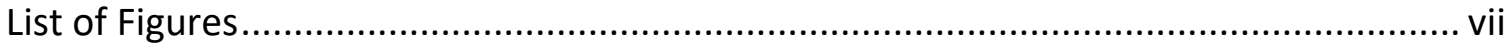

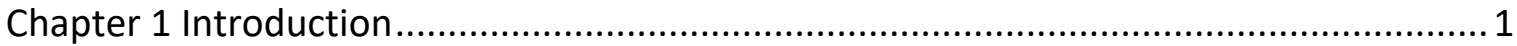

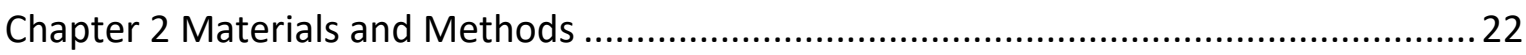

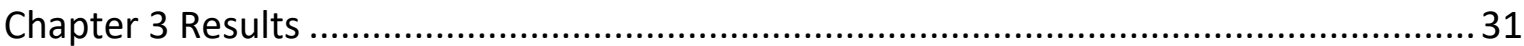

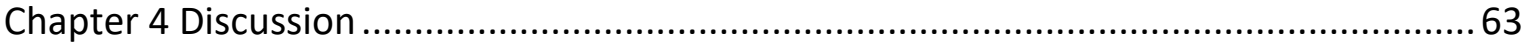

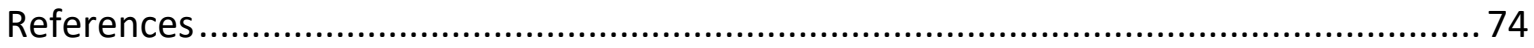

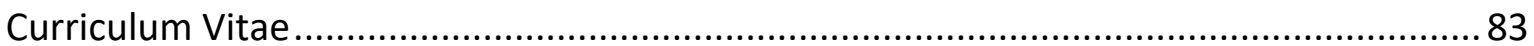




\section{LIST OF FIGURES}

Figure

Page

1. Summary of $P$. gingivalis virulence factors involved in host signaling manipulation and their targets

2. Potential mechanisms by which $P$. gingivalis could impact cancer associated processes in gingival epithelial cells .7

3. Schematic representation of polymicrobial synergy and dysbiosis model for oral squamous cell carcinoma . 20

4. Antagonistic interactions of $S$. gordonii on $P$. gingivalis through activation of the TAK1-NLK host kinase cascade

5. RNAseq of TIGK cells challenged with P. gingivalis and/or S. gordonii...... .37

6. Regulation of OLFM4 by $P$. gingivalis 39

7. Regulation of Notch signaling by $P$. gingivalis .

8. P. gingivalis induces paracrine activation of Notch signaling through soluble Jagged 1

9. Wnt/ $\beta$-catenin and ZEB2 are not required for activation of OLFM4 by $P$. gingivalis .45

10. SMAD3 enhances upregulation of Jagged1 by $P$. gingivalis .46

11. Activation of Notch signaling by $P$. gingivalis gingipains .48

12. Antagonism of P. gingivalis induced OLFM4 by S. gordonii........ 51

13. NLK-FOXO1 signaling is not required for inhibition of OLFM4 .54 
14. S. gordonii cannot inhibit Notch signaling activated with exogenous Jagged 1 .........55

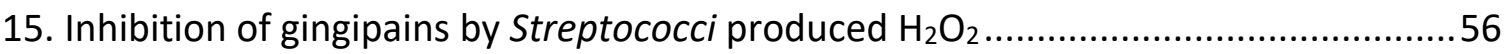

16. Knocking down OLFM4 reveals altered inflammatory transcriptomic profile...........60

17. P. gingivalis promotes migration of epithelial cells through OLFM4 .......................61

18. Graphic summarizing the pathway by which P. gingivalis activates Notch signaling,

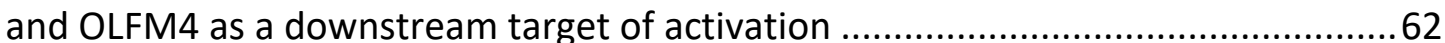

19. Graphic summarizing signaling pathways manipulated by $P$. gingivalis....................70

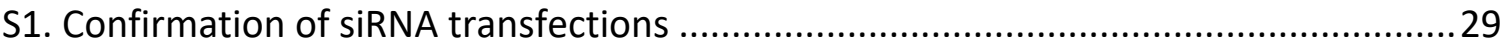




\section{CHAPTER 1 INTRODUCTION}

Worldwide, approximately 405,000 patients are diagnosed with oral cancer each year with a the 5 -year survival rate of just $50 \%$ [1]. This poor prognosis can be partially explained by the characteristic asymptomatic presentation in the early stages; by the time the patient has developed painful symptoms the carcinoma is in its late stages [2]. Oral cancers can originate as lymphomas in the lymphatic tissue of the tonsils and base of tongue; as carcinomas within salivary gland tissue; but most commonly as squamous cell carcinomas in areas of the mouth containing stratified squamous epithelium. Carcinomas of the oropharynx (including the base of the tongue) are generally referred to as oropharyngeal squamous cell carcinomas (OPSCC), and in OPSCC human papillomavirus (HPV) infection is a major risk factor [3]. Cancers of the oral squamous cells (OSCC), which account for about $90 \%$ of oral carcinomas, present most frequently on the tongue, lips, floor of the mouth, and gingiva [4]. Around $75 \%$ of OSCC can be attributed to tobacco smoking, which increases the risk for developing oral cancer by 6-fold [2]. Smoking also provides an encouraging environment for periodontal pathogens, and is an independent risk factor for periodontal disease [3]. Alcohol is another significant risk factor for oral cancer, and the combined risk for those who smoke and drink is increased fifteen-fold [2]. Gingival squamous cell carcinoma (GSCC) is particularly interesting because the traditional risk factors of smoking and alcohol consumption are not associated with this malignancy, and as the lesions mimic the appearance of periodontal disease they tend to 
go untreated [5]. Tooth loss as a result of bone loss in periodontal disease is an independent risk factor for head and neck, gastric, and colorectal cancer $[6,7]$. Additionally, colonization by periodontal pathogens has recently been identified as a risk factor for OSCC independent of alcohol, smoking, and HPV [3], and increased colonization by the periodontal pathogen Porphyromonas gingivalis has been correlated with GSCC [8]. The association between the oral microbiota (the collection of microorganisms found in the oral cavity) and cancers of the head and neck region has been extensively studied in recent years, using both culture dependent and independent methodology $[9,10]$. What has emerged is a picture of enrichment of particular organisms such as Fusobacterium nucleatum, Treponema denticola, and $P$. gingivalis, along with a decrease in the oral streptococci (with the exception of S. anginosus) $[9,10]$.

The notion that bacteria may be involved in the development of oral tumors is not new. For example Treponema pallidum was considered an etiological agent of OSCC back in the early part of the $20^{\text {th }}$ century. However, with the subsequent recognition of the importance of viruses in carcinogenesis the idea fell in to abeyance, and it was not until Helicobacter pylori was established as a cause of gastric cancer in the 1990s that the potential for a carcinogenic role of bacteria became rehabilitated [11, 12$]$. Mechanistically, there are a number of broadly defined categories by which bacteria could contribute to tumor growth and development. These include modulation of the balance of host cell proliferation and death; disruption of immune surveillance; and alteration of the metabolism of host produced compounds, nutritional substrates or pharmaceuticals [13]. Oral bacteria such as $P$. gingivalis, F. nucleatum and T. denticola exhibit properties 
consistent with these criteria, and can increase epithelial cell proliferation while inhibiting apoptosis, alter the inflammatory microenvironment and produce carcinogenic metabolites. These organisms will be discussed and referenced below due to their positive correlation with OSCC, as well the availability of mechanistic studies that have shown cancer-associated phenotypes.

\section{Porphyromonas gingivalis}

P. gingivalis is a keystone pathogen in periodontitis $[14,15]$ and many of the pathogenic mechanisms that impinge upon tissue integrity and disrupt protective immune responses are potentially relevant to tumorigenesis. These pathogenic mechanisms are summarized in Fig. 1.

Moreover, immunohistochemistry and other detection methods have identified increased colonization of $P$. gingivalis in OSCC, esophageal squamous cell carcinoma (ESCC), and GSCC $[8,16,17]$. In vitro, $P$. gingivalis engages gingival epithelial cells (GECs) in a complex molecular dialogue, a major thread of which involves subversion of host signaling pathways by bacterial effectors, such as the FimA-component fimbriae and the SerB serine phosphatase, to promote bacterial entry, intracellular trafficking and survival [18-20]. Comprehensive analyses of host transcriptional response to $P$. gingivalis invoke a pattern of enhanced cell survival and proliferation [21-23] phenotypes that have been verified by a number of laboratories. 


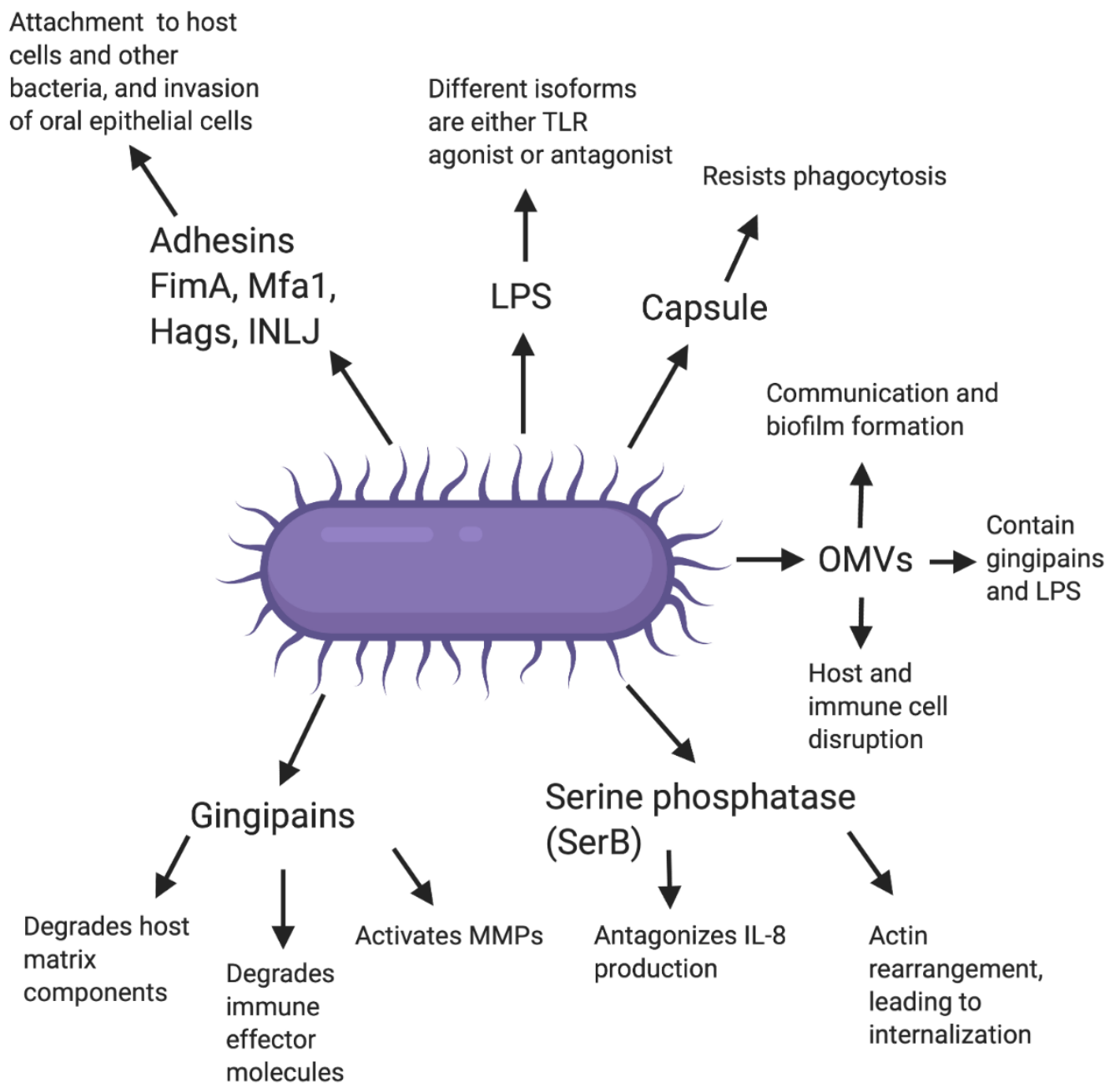

Figure 1: Summary of $P$. gingivalis virulence factors involved in host signaling manipulation and their targets.

Indeed, $P$. gingivalis utilizes multiple mechanisms to suppress host cell death and stimulate proliferation. In primary cultures of GECS, $P$. gingivalis activates the Jak1/Akt/Stat3 signaling hub that controls intrinsic mitochondrial apoptosis pathways [24, $25]$, and at the mitochondrial membrane the activity of proapoptotic effectors such as Bad is reduced, while the ratio of antiapoptotic factors, such as $\mathrm{Bcl} 2$, to proapoptotic 
factors is increased [26]. Downstream caspases including caspase-9 and the executioner caspase-3 are consequently suppressed. Additionally, $P$. gingivalis can modulate expression of microRNAs (miRs) in epithelial cells, and up-regulation of miR-203 leads to reduction of the proapoptotic signaling molecule SOCS3 [27]. A major antiapoptotic effector molecule of $P$. gingivalis is the secreted enzyme nucleoside diphosphate kinase (NDK), which can function as an ATPase and prevent ATP-dependent apoptosis mediated through the purinergic receptor P2X7 [28]. Another antiapoptotic function of NDK involves phosphorylation of Heat-shock-protein-27 (HSP27) which curtails cytochrome C release and caspase-9 activation [29]. Recently it has become apparent that $P$. gingivalis possesses a variety of kinase and phosphatase enzymes, some of which can function within host cells. Of particular relevance to cell survival, activation of the multi-purpose transcriptional regulator FOXO1 by dephosphorylation of serine residues induces antiapoptotic programs in epithelial cells, and knockdown of FOXO1 abrogates $P$. gingivalis-induced resistance to cell death [30].

Along with prolonged cell survival, increased proliferation is a feature of $P$. gingivalis infected epithelial cells. Signaling induced by the FimA fimbrial protein accelerates progression of primary GECs through the S-phase of the cell cycle by manipulation of cyclin/CDK (cyclin-dependent kinase) activity and by reducing the level of the p53 tumor suppressor [31]. The gingipains of $P$. gingivalis may also contribute to cell proliferation through proteolytic activation of $\beta$-catenin and disassociation of the $\beta$ catenin destruction complex. The accumulation of active $\beta$-catenin fragments in the nucleus drives the activity of the $\beta$-catenin-dependent, pro-proliferative TCF/LEF 
promoter [32]. In oral squamous carcinoma cells, $P$. gingivalis can increase cell proliferation by regulating cyclin D1 expression through the miR-21/PDCD4/AP-1 negative feedback signaling pathway [33]. Additionally, in oral tumor cells $P$. gingivalis can increase expression of $\alpha$-defensins which have been found to elevate proliferation through effects on epidermal growth factor receptor (EGFR) signaling [34].

Another role for $P$. gingivalis in carcinogenesis relates to its ability to influence the epithelial to mesenchymal transition (EMT) in GECs, as shown in Fig. 2. EMT is a cellular program through which epithelial cells shed their tight junctions in favor of an individual, mesenchymal phenotype. EMT is important for embryogenesis, and wound healing, but, if uncontrolled, ultimately leads to increased migration/invasion and cancer cell stemness. As befits its importance to the cell, EMT is controlled by a complex regulatory network involving a series of transcription factors such as Zinc Finger E-Box Binding Homeobox 1 (ZEB1), Zinc Finger E-Box Binding Homeobox 2 (ZEB2), Snail Family Transcriptional Repressor 1 (SNAI1), and Twist Family BHLH Transcription Factor 1 (TWIST1). These factors induce EMT by downregulating epithelial cell tight junction proteins (e.g. E- cadherin (CDH1), Zona Occludens Protein 1 (ZO-1)) while upregulating mesenchymal characteristics (e.g. $\mathrm{N}$-cadherin ( $\mathrm{CDH} 2)$, matrix metallopeptidase (MMP) 9 Vimentin (VIM)) [17, 35-38]. 


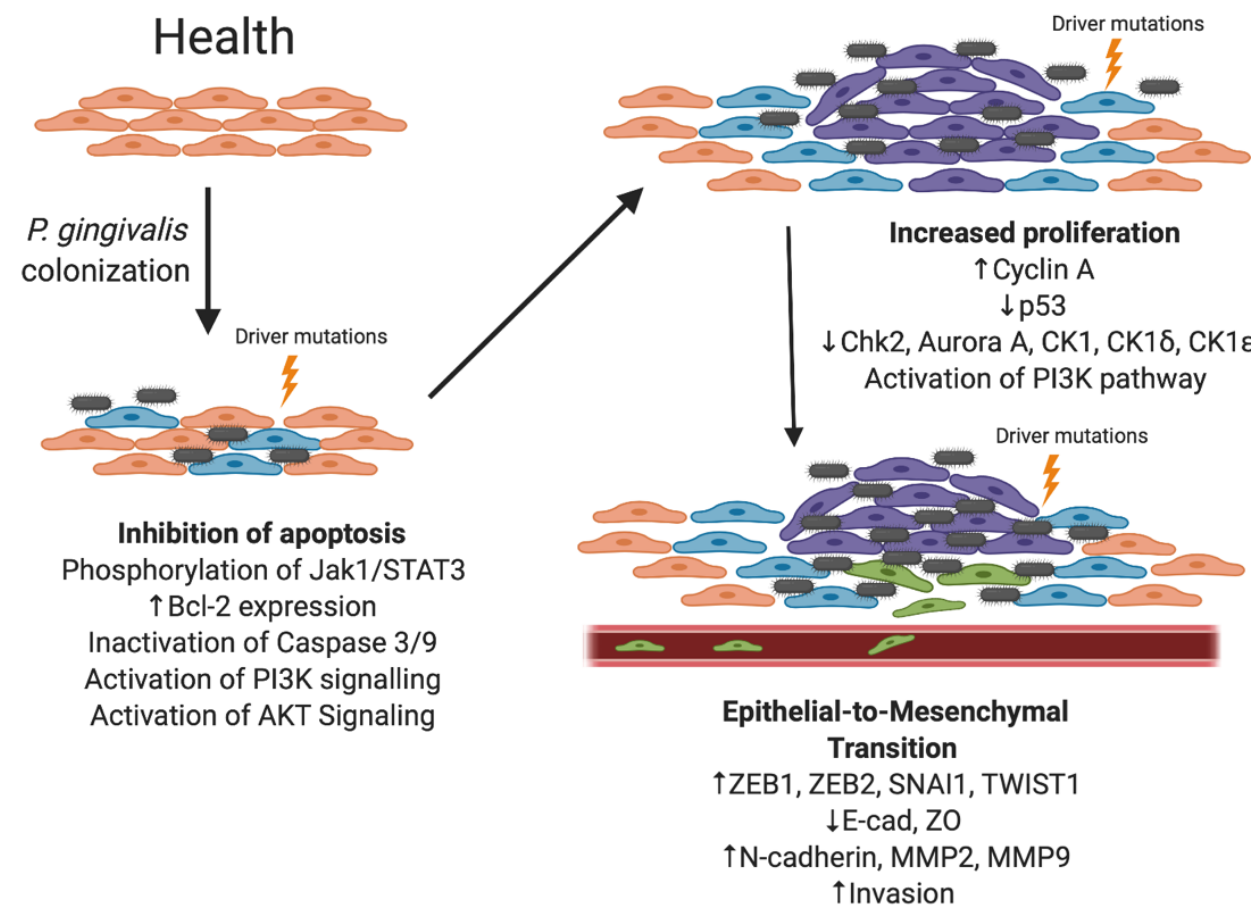

Figure 2: Potential mechanisms by which $P$. gingivalis (dark green rods) could impact cancer associated processes in gingival epithelial cells. Peach healthy epithelial cells, Blue epithelial cells that have acquired an anti-apoptotic phenotype, Purple epithelial cells that have acquired an accelerated proliferation phenotype, and Green epithelial cells that have undergone EMT, and have acquired an invasive phenotype. For simplicity other contributing host and environmental factors are not depicted.

ZEB1 is upregulated in a FimA-dependent manner through a pathway that involves GSK-3 $\beta$ in primary GECS $[17,37]$. ZEB2, on the other hand, is regulated in a FimAindependent manner involving gingipain processing and activation of $\beta$-catenin along with dephosphorylation and activation of FOXO1 [38]. Although the extent and duration of EMT induced by $P$. gingivalis remains to be determined, epithelial cell infection leads to an increase in stemness, as evidenced by upregulation of the stem cell markers CD44 and CD133 and enhanced migration [17, 35-38]. Invasion, and potentially metastasis of 
epithelial cells, can be facilitated by host MMP enzymes which degrade extracellular matrix and basement components. $P$. gingivalis has been shown to upregulate production of several MMPs including MMP-1, MMP-2, MMP-7, MMP-9 and MMP-10 from primary and transformed oral epithelial cells [17, 36, 37, 39, 40]. In invasive OSCC lines, $P$. gingivalis gingipains can stimulate proteinase-activated receptor 2 (PAR2) and PAR4 to increase MMP-9 proenzyme expression through ERK1/2-Ets1, p38/HSP27, and NF-kB pathways [40]. Subsequently, in a two-hit mechanism, gingipains process the proenzyme to active MMP9 ensuring an increase in cellular invasion $[40,41]$

P. gingivalis has also been shown to activate Notch signaling through its secreted gingipains. Notch signaling is another pathway that can be activated to inhibit apoptosis, accelerate cell cycle progression, and induce EMT. Al-Attar et al. have shown that $P$. gingivalis activates Notch signaling specifically through Notch1, and the triple gingipain knockout mutant was unable to stimulate the pathway [42]. These investigators primarily focused on Notch activation leading to secretion of PLA2-IIA, which is an anti-microbial peptide utilized by $P$. gingivalis to shift to a more pathogenic microbial community [42].

While a role for $P$. gingivalis induced Notch signaling has been defined in terms of anti-microbial peptide regulation, traditional Notch regulated phenotypes associated with transformation of epithelial cells have not been characterized. Notch signaling has also been shown to regulate Olfactomedin 4 (OLFM4), which is a secreted glycoprotein that was first characterized by its role in inhibition of apoptosis through binding of the mitochondrial protein GRIM19 [43]. Since that initial study, OLFM4 has been shown to 
additionally accelerate cell-cycle progression, activate EMT, positively regulate MMPs, and dampen the innate immune response [44-47].

Liu et. al. showed that $H$. pylori upregulates OLFM4 in the stomach, and this regulation allows for efficient colonization [47]. Moreover, upon deletion of OLFM4, $\mathrm{H}$. pylori was efficiently cleared by an unimpaired innate immune response. Another study sought to characterize the secretome of non-neoplastic, immortalized epithelial cells and neoplastic oral derived cell lines [48]. OLFM4 was the most secreted protein (12-fold increase) in tested supernatants of neoplasm-derived cell lines compared to the supernatant of non-neoplasm, immortalized cells, although its extracellular function has not been characterized [48] .

Chronic inflammation has emerged as a major contributor to tumor growth and metastasis, mainly through modulation of the tumor microenvironment by cytokines and chemokines, and through differential receptor expression [49]. The ability of $P$. gingivalis to incite prolonged, dysregulated inflammation could also contribute to the epidemiological associations between periodontitis and $\operatorname{OSCC}[49,50]$. In both OSCC cell lines and primary GECS $P$. gingivalis can upregulate programmed death-ligand 1 (PD-L1, B7-H1) and B7-DC, receptors that lead to anergy and apoptosis of activated T cells, and contribute to tumor cells' resistance to host immune responses [51]. In OSCC cells $P$. gingivalis stimulates the release of a variety of chemokines/cytokines including IL-8, IL-6, TGF- $\beta 1$, and TNF- $\alpha[35,39,52]$. IL-8 can increase MMP production and cell invasiveness, as well as stimulate proliferation through transactivation of the Epidermal growth factor (EFG)receptor [53]. In addition, the IL-23/IL-17 axis, which is strongly pro-tumorigenic in 
colorectal cancer [54], can be induced by $P$. gingivalis [55]. Interestingly, in primary GECs P. gingivalis adopts a more stealth-like behavior through a process known as localized immune paralysis [56]. Release of a serine phosphatase (SerB) intracellularly results in antagonism of IL-8 production through dephosphorylation of the serine 538 residue of the p65 subunit of NF-KB [57]. Dephosphorylation of the p65 subunit prevents dimerization of the subunits, thus inactivating NF-KB. While this may restrain tumor progression, the effect may be offset by inhibition of the angiostatic chemokines CXCL9, CXCL10 and CXCL11 that would otherwise promote neovascularization of tumors and increased tumor growth or metastasis $[49,58]$.

In vivo evidence also supports a role for $P$. gingivalis in the development of oral carcinomas. In the 4-nitroquinoline-1-oxide (4NQO) tongue squamous cell carcinoma model, $P$. gingivalis-treated mice developed more and larger tumors on the tongue compared to the carcinogen alone group [59-62]. The development of squamous cell carcinoma was associated with enhanced free fatty acid production both in the tongue and in the serum of $4 \mathrm{NQO}$ treated mice, which is a shift also seen in oral cancer.

\section{Fusobacterium nucleatum}

While $F$. nucleatum is prevalent in a healthy microbiota, several studies have found that $F$. nucleatum is significantly enriched in patients with disease, whether that be periodontal disease, preterm delivery of low birth weight infants, head and neck cancer, or colorectal cancer (CRC) [63]. The potential role of $F$. nucleatum in cancers has been investigated in both in vitro and in vivo studies. F. nucleatum produces an adhesin, FadA, which is crucial for attachment and subsequent invasion of epithelial cells [64]. FadA is 
thought to play a major role in CRC by binding to E-cadherin on CRC cells, thus activating $\beta$-catenin signaling and differentially regulating inflammatory and oncogenic responses [65]. The FadA-E-cadherin axis also upregulates Annexin A1, a modulator of Wnt/Bcatenin-based proliferative signaling in CRC cells [66]. Localization of what is primarily an oral organism with developing tumors in the GI tract may be accomplished by another fusobacterial adhesin, Fap2, which binds to Gal-GalNac, abundant on CRC cell surfaces [67]. Fap2 can also immunosuppress tumor infiltrating lymphocytes, which are essential for immune responses to tumors. Specifically, Fap2 binds and activates the inhibitory immunoreceptor TIGIT which is expressed by T and Natural Killer (NK) cells [68]. Further compromising anti-tumor immunity, F. nucleatum activates the human inhibitory receptor CEACAM1 which also suppresses T and NK cells activities [69]. Clearly then, $F$. nucleatum can significantly impact cell signaling and tumor immunity with relevance to CRC. The extent to which these properties may pertain to OSCC is a vein of information ready to be mined. Studies that have been performed establish the ability of $F$. nucleatum to induce nuclear localization of NF-KB in GECs, while also increasing secretion of IL-1 $\beta$ via activation of the NLRP3 inflammasome and caspase-1 [70]. Release of endogenous danger-associated molecular patterns (DAMPs) such as apoptosis-associated speck-like protein (ASC) and high-mobility group box 1 protein (HMGB1) further amplifies inflammation. P38 is also activated by F. nucleatum, which leads to increased secretion of MMP9 and MMP13 [71]. Moreover, F. nucleatum can induce an EMT program in OSCC cells through upregulation of TGF- $\beta$, TNF $\alpha$, and EGF signaling [35]. In conclusion, $F$. 
nucleatum induces proliferation of CRC cells, suppresses T and NK cell activity, and increases secretion of MMP9 and MMP13, all of which contribute to carcinogenesis.

Coinfection with $F$. nucleatum and $P$. gingivalis exacerbated in vivo tumor development in the murine $4 \mathrm{NQO}$ tongue squamous cell carcinoma model [60]. The infected group had larger, more invasive tumors, with increased expression of cell cycle progression marker Cyclin D1 [60]. There was also an increase in phosphorylation of STAT3 in the infected group, which led to increased expression of IL-6 [60]. Further, in a colorectal cancer model $F$. nucleatum increases the size and number of tumors which develop in $\mathrm{C} 57 \mathrm{BI} / 6 \mathrm{ApC}^{\mathrm{min} /+}$ mice [72]. In summary, F. nucleatum enhances tumorigenesis in OSCC and CRC murine models, and with P. gingivalis is of critical importance in understanding microbial contributions to oral carcinogenesis.

\section{Treponema denticola}

In a healthy person's oral cavity, $T$. denticola is found in low abundance; however, in periodontal disease, $T$. denticola is one of the most abundant organisms [73]. Similarly, an increased abundance of $T$. denticola has been associated with ESCC and OSCC, and similarly correlated with an increased risk of CRC [74]. T. denticola is highly proteolytic, with dentilisin (chymotrypsin-like proteinase) being the primary secreted protease [75]. The presence of dentilisin is strongly correlated with early-stage mobile tongue squamous cell carcinoma, and high expression of dentilisin is associated with increased tumor invasion, tumor size, and recurrence in patients less than 60 years old [76]. Dentilisin can degrade IL-8 and TNF $\alpha[77,78]$, and cleaves pro-MMP8 and pro-MMP9 to their active forms [10]. In a two-hit mechanism, dentilisin also degrades tissue inhibitors of MMPs, 
TIMP1 and TIMP2, contributing to overall more proteolytic environment favoring invasion of epithelial cells. To summarize the above, T. denticola is associated with ESCC, OSCC, and CRC, and its secreted protease dentilisin is responsible for degradation of IL-8, TNF $\alpha$, TIMP1, and TIMP2, all of which could contribute to an environment capable of inducing transformation of epithelial cells.

\section{Oral Microbial Communities}

The preceding text describes how individual species impact carcinogenesis; however, in the oral cavity, bacteria assemble into multi-species, spatially constrained communities known as biofilms. Within these communities, functional specialization of bacterial species emerges, and in periodontitis it is pathogenicity at the community level, or nososymbiocity, which is thought to determine the potential for disease [15]. Metabolic interactions can drive the spatial and temporal arrangement of organisms in the oral polymicrobial community, and individual species tend to associate with partners that are physiologically compatible [79]. Interestingly, a bioinformatics study of 11 oral bacteria identified a large redundancy in the metabolic potential of the community, and metabolic capabilities varied among early and late colonizers [80]. Furthermore, a recent meta- transcriptomic analysis of diseased periodontal sites as compared with patient matched healthy sites determined the metabolic profile of the community was highly conserved, whereas individual species associated with this profile were interchangeable. In other words, the overall metabolic profile is more consistent than the presence or absence of individual species. These studies suggest that the overall metabolic potential

of the community may correlate better with disease than the presence of individual 
species [81]. Overall metabolic stability is likely driven by shared ecology and stresses, and community homeostasis is a balance between metabolic redundancy and metabolic cross-feeding. If the metabolic capabilities and community metabolic necessities overlapped to a large extent, this would result in constant competition among individual species for nutrients. Instead, the conservation of core metabolic functions and mutualistic metabolism reduces antagonistic interactions between integral community participants and promotes community homeostasis.

\section{Spatial Determinants Between Oral Microbes}

Within a subgingival biofilm, the stable environment and intimate proximity facilitate nutrient transfer mechanisms among microbial constituents. For example, spatial imaging of plaque biofilms shows that Porphyromonas gingivalis and Fusobacterium nucleatum are often in close association [82]. In addition, F. nucleatum enhances the growth of $P$. gingivalis in the presence of oxygen, which suggests that the more aerotolerant $F$. nucleatum consumes oxygen, thereby providing a microenvironment with lower redox potential to benefit the growth of more oxygensensitive organisms [83].

In a study by Palmer et al. [84], Actinomyces naeslundii was incubated in minimal medium that did not support its growth; however, it displayed robust growth and coaggregation when cocultured with Streptococcus oralis. Streptococcus gordonii and related mitis group streptococci participate in several instances of cooperative metabolism. S. gordonii catabolizes carbohydrates and secretes $\mathrm{H}_{2} \mathrm{O}_{2}$ and L-lactate, a major carbon source for lactate-utilizing bacteria such as Veillonella atypica and 
Aggregatibacter actinomycetemcomitans [85, 86]. Consequently, S. gordonii and related organisms can promote $A$. actinomycetemcomitans growth, biofilm formation, and in vivo pathogenicity $[87,88]$. V. atypica induces expression of the amy $B$ gene, encoding an $\alpha-$ amylase, of S. gordonii [89]; and thus S. gordonii grows better in association with $V$. atypica and secretes more lactate due to carbohydrate utilization, to the benefit of both organisms. Moreover, cooperative metabolism may have global benefits to other community members, as the lactate-utilizing $V$. atypica and A. actinomycetemcomitans neutralize acidification of the oral environment, thus protecting more acid-sensitive species, such as $P$. gingivalis.

\section{Metabolic Cooperation between A. actinomycetemcomitans and S. gordonii}

The relationship between $A$. actinomycetemcomitans and $S$. gordonii is more complex and involves a balance between synergistic cross-feeding and antagonistic interactions. Stacy et al. [90] demonstrated that A. actinomycetemcomitans spatially positions itself far enough away to allow detoxification of $\mathrm{H}_{2} \mathrm{O}_{2}$ through the production of catalase, but close enough to S. gordonii to benefit from the secretion of L-lactate. Optimal positioning of $A$. actinomycetemcomitans within the biofilm matrix is achieved through the production of dispersin $B$, an enzyme that hydrolyzes polysaccharides and is upregulated in the presence of S. gordonii [91]. Additionally, S. gordonii increases the availability of oxygen for $A$. actinomycetemcomitans to use as a terminal electron acceptor. In this way, S. gordonii shifts the metabolism of A. actinomycetemcomitans from fermentative to oxidative respiration [92]. This process, termed "cross-respiration," also enhances the growth and fitness of $A$. actinomycetemcomitans. Similar homeostatic 
mechanisms to maximize energy gains from metabolic cross-feeding, while reducing antagonistic interactions, may represent a fundamental force in shaping the spatial organization of dental plaque. A. actinomycetemcomitans possesses the capability to influence global regulation of community development, and its presence in an in vitro multispecies biofilm was shown to modulate the proteomic profile of the entire complex community [95]. Further investigation identified the community regulator as the histonelike family of nucleoid-structuring (H-NS) proteins of A. actinomycetemcomitans. H-NS proteins act as translational silencers with global regulatory potential in many Gramnegative bacteria. In Escherichia coli, deletion of the hns gene attenuates biofilm development [93], similar to its role in A. actinomycetemcomitans, where it promotes biofilm development as well as adhesin production [94]. Proteomic analysis of an in vitro polymicrobial community with the hns deletion strain of $A$. actinomycetemcomitans suggested that H-NS globally regulates community metabolic pathways involved in peptide, carbohydrate, and malate metabolism [95].

\section{Metabolic Cooperation Between Oral Streptococci and P. gingivalis}

Oral streptococci also have a significant impact on the properties and pathogenic potential of $P$. gingivalis, and these organisms are located in close proximity in vivo [82]. Interactions occur on multiple levels and many involve metabolic communication. Oral streptococci efficiently metabolize sugars, and a reduction in galactose in the biofilm milieu could alter physiology, cell shape, and intracellular granulation of $P$. gingivalis [96].

S. gordonii promotes increased biofilm development and synergistic pathogenicity in rodent models of periodontitis with $P$. gingivalis [97-99]. The $S$. gordonii genes spxB and 
cbe are among those instrumental in promoting biofilm development with $P$. gingivalis [100]. SpxB (pyruvate oxidase) produces acetyl phosphate and $\mathrm{H}_{2} \mathrm{O}_{2}$ from pyruvate under aerobic conditions, but its role in promoting biofilm development with $P$. gingivalis remains unclear. Cbe (chorismate- binding enzyme) synthesizes para-aminobenzoic acid (pABA) for secretion and folate biosynthesis. Recently, pABA was determined to promote fitness and colonization of $P$. gingivalis in vivo with a mouse oral model [101]. Moreover, pABA reduced the overall stress of $P$. gingivalis while promoting fimbrial production. Metabolic and proteomic analyses of $P$. gingivalis responses to pABA showed that $P$. gingivalis can utilize pABA for folate biosynthesis. Surprisingly, pABA reduced the production of exopolysaccharide and attenuated the virulence of $P$. gingivalis. These metabolic interactions may regulate community density and spatial configuration, again supporting the developing narrative that metabolic communication promotes structure and polymicrobial synergy within the oral biofilm [102]. It is important to note that individual species of oral streptococci can affect $P$. gingivalis synergistically or antagonistically through distinct process. For example, Streptococcus cristatus and Staphylococcus intermedius are antagonistic to $P$. gingivalis $[103,104]$, in part through removal of the biofilm-promoting amino acid arginine by streptococcal arginine deiminase enzymes [103].

\section{T. denticola and $\boldsymbol{P}$. gingivalis Metabolic Interactions}

Most of the examples discussed so far involve early colonizing commensal bacteria and more pathogenic later colonizers; however, interactions among these secondary colonizers are also manifold [105]. One example of cooperative metabolism between 
periodontal pathogens is the metabolic cross-talk between Treponema denticola and $P$. gingivalis. When cultured together, P. gingivalis and $T$. denticola coaggregate and display enhanced growth [106-108]. Gas-liquid chromatography analysis of culture supernatants demonstrated that $P$. gingivalis produces isobutyric acid, which stimulates the growth of T. denticola, while $T$. denticola secretes succinate, which is utilized by $P$. gingivalis [106]. Additional studies demonstrated that $T$. denticola induces the production and secretion of glycine by $P$. gingivalis to promote its own growth in mixed culture [107]. Transcriptome analysis also suggests that $P$. gingivalis produces thiamine pyrophosphate, an essential nutrient for T. denticola; however, direct cross-feeding of this metabolite has yet to be established. Recently, in vivo metatranscriptomic studies showed that these cooperative metabolic interactions occur during periodontitis and may be a significant contributor to synergistic pathogenicity $[109,110]$.

\section{Polymicrobial Synergy and Dysbiosis in Cancer}

One current theory of periodontal disease etiology, the polymicrobial synergy and dysbiosis (PSD) model, holds that synergistic interactions within the polymicrobial community shape and stabilize a dysbiotic microbiota which perturbs host homeostasis. Disease is caused by reciprocally reinforced interactions between such physically and metabolically integrated polymicrobial communities and a dysregulated host inflammatory response [102]. While periodontitis and cancer are clearly distinct diseases, they share an underlying similarity in that they are, in essence, wounds that fail to heal [111]. Community perturbations consistent with a PSD model have been proposed for tumor development $[112,113]$. Combinations of oral bacterial species are consistently 
identified in OSCC lesions, resonating with the idea that community-wide properties may promote tumorigenesis.

In the 4NQO model, F. nucleatum and P. gingivalis synergistically promote cancer progression [60]. Another feature of the PSD model is that the microbial roster is of less relevance to nososymbiocity than the presence of combinations of functional genes, as communities of different compositions can exhibit similar functions [102]. Support for this concept in OSCC comes from a study of the microbiota and transcriptome in the 4NQO mouse model. Whereas variability in community dynamics was observed, the metatranscriptome revealed patterns of metabolic signatures consistently present in OSCC. These include nitrogen transport, response to stress, interspecies interactions, Wnt pathway modulation, and amino acid and lipid biosynthesis [62]. Similarly, a pilot study of human OSCC tumors found metabolic activities better correlated with disease than did community microbial composition [114], and a comparison of microbiotas associated with OSCC in different countries reveals functional rather than compositional similarities [115]. Fig. 3 summarizes a proposed role for PSD in OSCC. 


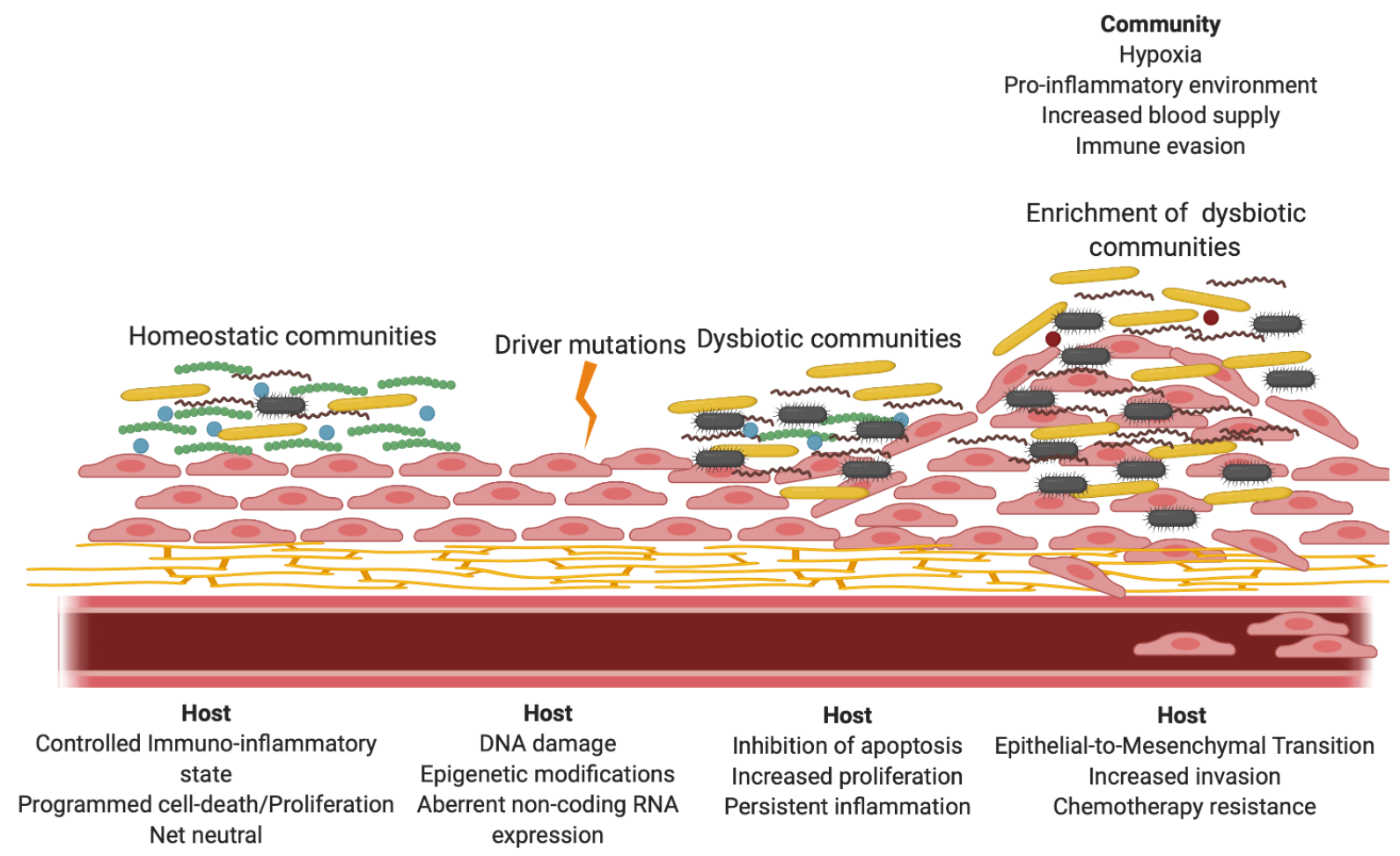

Figure 3: Schematic representation of polymicrobial synergy and dysbiosis model for oral squamous cell carcinoma. In health, host cell responses to homeostatic communities are a eubiotic balance of proliferation with programmed cell death. Driver mutations lead to dysregulation of host processes, which can also be manipulated by organisms associated with a dysbiotic community. As the tumor microenvironment is established anaerobic, gram-negative organisms are enriched as a result of hypoxic, pro-inflammatory conditions. As organisms such as $P$. gingivalis, F. nucleatum and $T$. denticola accumulate, the tumor cells can acquire an invasive phenotype through EMT, as well as increased resistance to chemotherapeutic drugs.

Interactions among bacterial constituents of communities can be antagonistic as well as synergistic, and numerous cases of antagonisms have been documented among oral bacteria $[38,102,116]$ Although there are conflicting reports in the literature [117], in general, most oral streptococcal species tend to be underrepresented in the microbiotas associated with OSCC $[62,118]$. This can be interpreted to indicate reduced fitness of these organisms in a tumorigenic environment. However, a further 
interpretation is that these organisms are eubiotic and help maintain homeostasis at mucosal membranes; in their absence the microbiota becomes increasingly tumorigenic. In that regard, S. gordonii can reprogram epithelial cell global transcriptional patterns such that the subsequent response to $P$. gingivalis is diminished, and, further, $S$. gordonii can prevent $P$. gingivalis-induced gingival epithelial cell proliferation [102]. Moreover, $S$. gordonii can antagonize P. gingivalis-induced ZEB2 production and associated cell migration by inhibiting the activation of the FOXO1 transcription factor through the TAK1NLK negative regulatory pathway, as shown in Fig. 4 [38]. Hence, while S. gordonii is an accessory pathogen in periodontal disease [102], this species may be a homeostatic commensal in oral cancer, an illustration of the importance of environmental context for bacterial functionality.

The goal of this study was to identify signaling events manipulated by periodontal pathogen $P$. gingivalis that contribute to homeostatic disruption, specifically focusing on pathways overridden by homeostatic commensal S. gordonii in gingival epithelial cells. We hypothesized that $P$. gingivalis would activate genes involved in tissue disruption and dampen innate immune signaling, whereas S. gordonii would either program the cells to resist this activation or interact with other inhibitory pathways. We characterized the differences in transcriptomic responses of epithelial cells challenged with $P$. gingivalis and/or S. gordonii using RNAseq. The transcriptome data revealed OLFM4, a gene involved in apoptosis, proliferation, migration, and dysregulation of innate immunity, all of which are differentially influenced by the microbes, as a potential axis that is disturbed by $P$. gingivalis and restored by $S$. gordonii. The initial identification of 
OLFM4 was followed by characterization of the mechanism for upregulation by $P$. gingivalis, inhibition by S. gordonii, and ultimately the phenotypic outcome of this differential regulation.

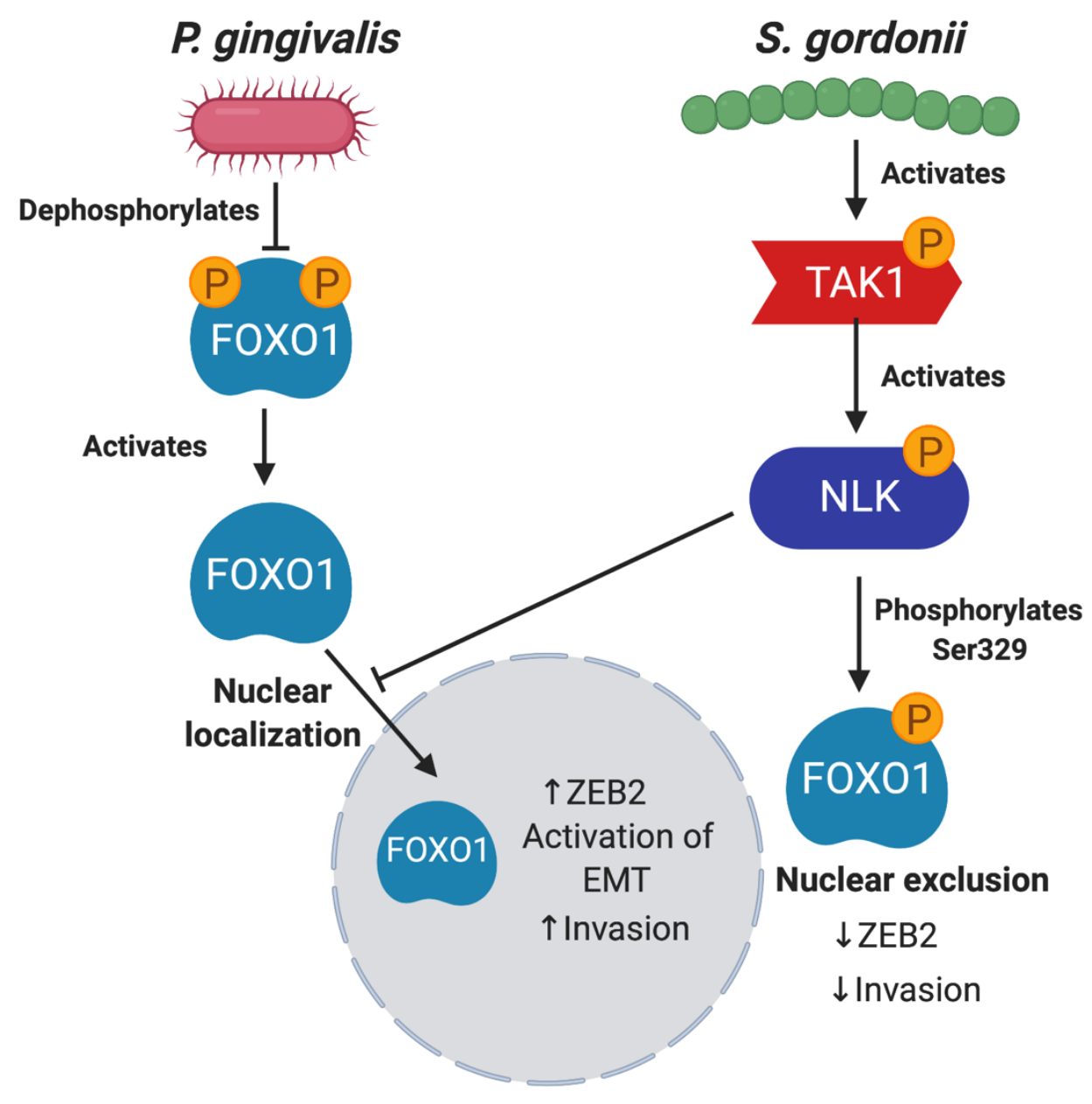

Figure 4: Antagonistic interactions of $S$. gordonii on $P$. gingivalis through activation of the TAK1-NLK host kinase cascade. $P$. gingivalis can dephosphorylate FOXO1 on serine residues which prevents translocation form the nucleus to the cytoplasm thus enhancing activity. When $S$. gordonii is present the TAK1-NLK1 pathway is activated which 
supersedes the effect of $P$. gingivalis and increases phosphorylation of FOXO1 on Ser329 thus allowing translocation of FOXO1 to the cytoplasm where it is inactive. 


\section{CHAPTER 2 MATERIALS AND METHODS}

Eukaryotic Cells, Bacterial Strains, Growth and Infection Conditions. Telomerase immortalized gingival keratinocytes (TIGKs) [119] and OKF6/TERT2 cells [120] were maintained with Dermalife-K Serum free culture medium supplemented with DermaLife K LifeFactors Kit. SCC9 cells from ATCC were grown in 1:1 DMEM: Ham's F12 medium supplemented with $400 \mathrm{ng} / \mathrm{ml}$ hydrocortisone and 10\% fetal bovine serum. ESCC9706 cells [121] were grown using DMEM supplemented with $10 \%$ fetal bovine serum. Cells were cultured at $37^{\circ} \mathrm{C}$ with $5 \% \mathrm{CO}_{2}$. Porphyromonas gingivalis ATCC 33277, HG66, W83, 381, A1A7-28, and low passage clinical isolate MP4-504 were grown in trypticase soy broth (TSB) supplemented with $1 \mathrm{mg} / \mathrm{mL}$ yeast extract, $5 \mu \mathrm{g} / \mathrm{mL}$ hemin and $1 \mathrm{ug} / \mathrm{mL}$ menadione. Deletional mutants $\Delta$ fimA, $\Delta m f a 1, \Delta \operatorname{ser} B$, and $\Delta$ pork $[57,122-124]$ were grown in the presence of $10 \mu \mathrm{g} / \mathrm{mL}$ erythromycin, $\Delta k g p$ was grown in the presence of 20 $\mathrm{ug} / \mathrm{mL}$ chloramphenicol, $\Delta / \mathrm{tp} 1 / \Delta p h p 1$ and $\Delta \operatorname{rgp} A / B$ were grown in the presence of erythromycin and $1 \mathrm{ug} / \mathrm{mL}$ tetracycline, and $\Delta \mathrm{kgp} / \mathrm{rgpA} / B$ were grown in the presence of erythromycin, tetracycline, and chloramphenicol [125]. Streptococcus gordonii DL1, S. mutans KSPK2, S. oralis 10557, S. constellatus ATCC 27823, and S. sanguinis 10556 were grown in brain heart infusion broth (BHI) supplemented with $5 \mathrm{mg} / \mathrm{ml}$ yeast extract, $S$. gordonii $\Delta s p x B C \Delta s p x B, \Delta s s p A / B, \Delta c b e,[100]$ were grown in the presence of $5 \mu \mathrm{g} / \mathrm{mL}$ erythromycin, and $C \Delta s p x B$ was grown with $10 \mu \mathrm{g} / \mathrm{mL}$ tetracycline. Fusobacterium nucleatum ATCC 25586 was grown in BHI supplemented with $1 \mathrm{mg} / \mathrm{mL}$ yeast extract, 5 
$\mu \mathrm{g} / \mathrm{mL}$ hemin, and $1 \mu \mathrm{g} / \mathrm{mL}$ menadione. Treponema denticola ATCC 35405 was grown in new oral spirochete broth (NOS), and Filifactor alocis ATCC 35896 was grown in BHI supplemented with $5 \mathrm{mg} / \mathrm{mL}$ yeast extract, $5 \mu \mathrm{g} / \mathrm{mL}$ hemin, $1 \mu \mathrm{g} / \mathrm{mL}$ menadione, and $20 \%$ $\mathrm{L}$ - arginine. All bacteria were grown to mid-log phase at $37^{\circ} \mathrm{C}$ under standard anaerobic conditions of $85 \% \mathrm{~N}_{2}, 10 \% \mathrm{H}_{2}, 5 \% \mathrm{CO}_{2}$.

Cells were grown to $~ 80 \%$ confluence and challenged with bacteria at an $\mathrm{MOI}$ of 100 for $1 \mathrm{~h}$, followed by $23 \mathrm{~h}$ culture in fresh medium, unless otherwise noted.

\section{Antibodies and Reagents}

OLFM4, Activated Notch1, and GAPDH antibodies were from Abcam. Phalloidin- Texas Red, Alexa Fluor 488, and DAPI were from Thermofisher. OLFM4 and Notch1 ELISAs were from Abcam, and Jagged1 ELISA was from Thermofisher. OLFM4, Notch1, Notch2, Notch3, Notch4, Jagged1, Jagged2, DLL1, DLL3, DLL4, SMAD3, SMAD7, ZEB2, FOXO1, $\beta$-catenin, GSK3 $\beta$, ADAM10, ADAM17, and NLK siRNA were from Origene. TLCK, FH535, and chromogenic substrate L-BAPNA were from Sigma Aldrich. Recombinant chimera Jagged1-FC was from RND systems. Gamma secretase inhibitor LY-374973 (DAPT) was from Sigma Aldrich, and ADAM/TACE inhibitor TAPI-2 was from Tocris.

\section{Quantitative Reverse Transcription-PCR}

Cells were harvested, and RNA was purified using RNAeasy plus kit from Qiagen. 2ug RNA was reverse transcribed using high capacity reverse transcription kit (Thermofisher). 
Applied Biosystems Taqman fast universal master mix and Taqman gene expression assays for OLFM4, GAPDH, HES5, Notch1, Notch2, Notch3, Notch4, Jagged1, Jagged2, DLL1, DLL3, DLL4, SMAD3, SMAD7, ZEB2, FOXO1, $\beta$-catenin, GSK3 $\beta$, ADAM10, ADAM17, and NLK were from Thermofisher. qRT-PCR was performed using an Applied Biosystems

QuantStudio 3. Thresholds were automatically calculated using Applied Biosystems software, and Cycle threshold (Ct) values were normalized to GAPDH. Fold changes were calculated using 2-(delta delta)Ct.

\section{Immunoblots and ELISAs}

Cells were lysed using RIPA buffer containing Protease and PhosSTOP phosphatase inhibitor (Roche). Protein concentration was estimated by a bicinchoninic acid (BCA) assay. Proteins $(20 \mu \mathrm{g})$ were separated on a 10\% SDS-PAGE gel, and transferred to a nitrocellulose membrane $(0.2 \mu \mathrm{m})$ by electroblotting for $1 \mathrm{~h}$ at $100 \mathrm{~V}$. Nitrocellulose membranes were blocked with $5 \%$ bovine serum albumin (BSA) in Tris buffered saline with $0.1 \%$ Tween 20 (TBST) for $1 \mathrm{~h}$ at room temperature. The membrane was probed with primary antibodies in TBST overnight at $4^{\circ} \mathrm{C}$. Membranes were washed 3 times with TBST, and probed with HRP-conjugated secondary antibody at room temperature for $1 \mathrm{~h}$. ECL substrate (ThermoFisher) was used to visualize the proteins, and images were generated using a ChemiDoc XRS Plus (BioRad). 
Cell culture supernatant was centrifuged for $20 \mathrm{~min}$ at $20,000 \times \mathrm{xg}$ at $4^{\circ} \mathrm{C}$, filtered $(0.4 \mu \mathrm{m})$, and concentrated using Amicon Ultra concentrators. For ELISA, $25 \mu$ l of supernatant per well was used per manufacturer's guidelines.

\section{Plasmid preparation, RNA interference, Transfections, and Luciferase assay}

OLFM4 promoter-luciferase reporter construct was made by amplification of a 500 bp fragment upstream of the OLFM4 coding sequence, which was cloned into pGL3-basic plasmid (Promega). All constructs were confirmed by sequencing at the University of Louisville Sequencing Core. The internal control reporter was $\mathrm{pRL}$ vector that provides constitutive expression of Renilla luciferase (Promega). For siRNA cells were grown to 50$60 \%$ confluence, and cells for plasmid transfection were grown to $60-70 \%$ confluence. Transfection was for $48 \mathrm{~h}$ using Lipojet transfection agent (SignaGen), and after $24 \mathrm{~h}$ medium was changed to allow cells to recover before bacterial challenge. Confirmations of knockdowns can be found in Fig. S1. Luciferase reporter assays were performed using a Stop \& Glo Dual luciferase reporter kit from Promega. Luciferase activity was measured using a 10s integration time in a Luminometer (Molecular Devices). Firefly luciferase activity was normalized to Renilla luciferase activity from the same lysates. 
A
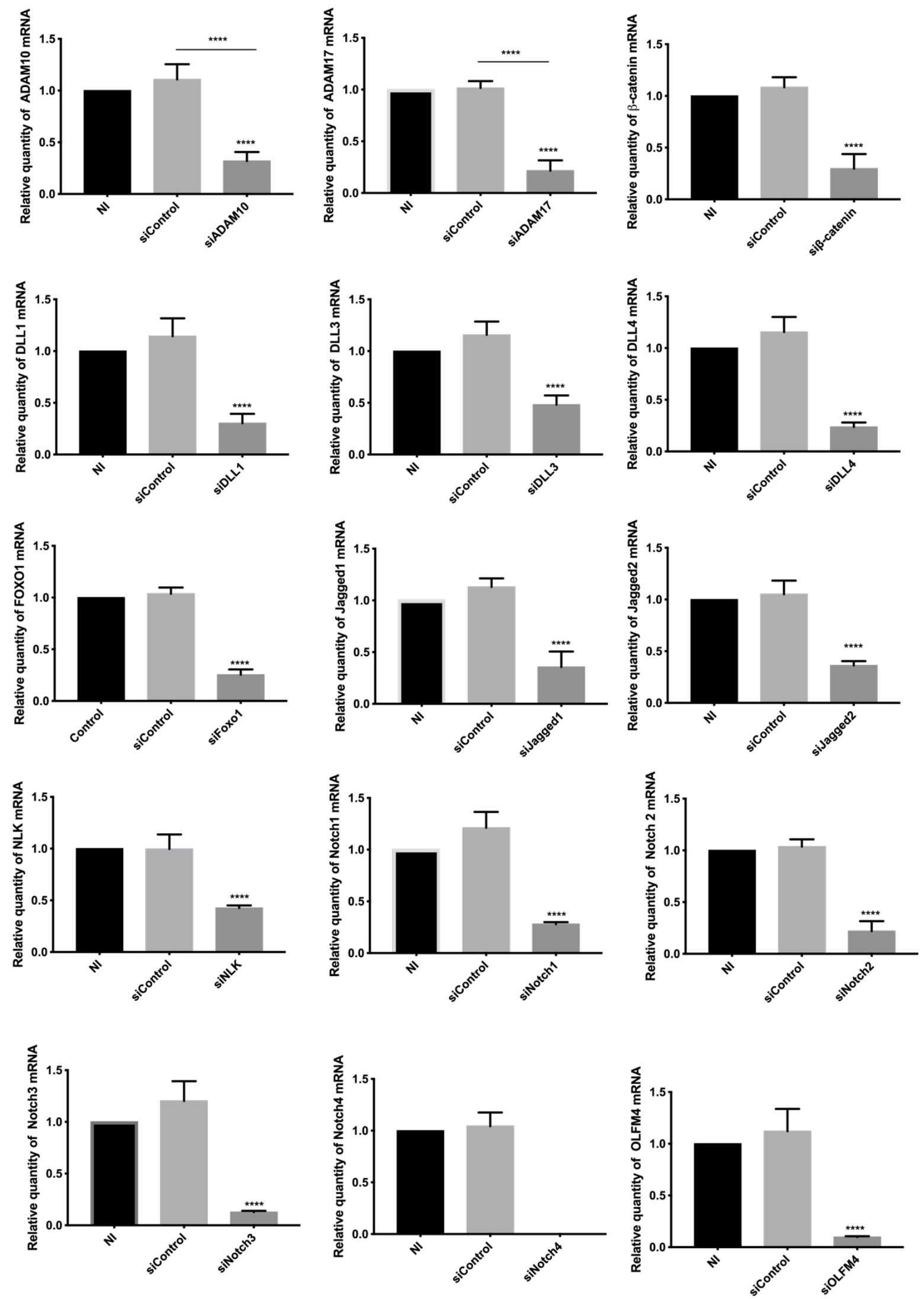
Figure S1: Confirmation of siRNA transfections. Target genes for qRT-PCR were normalized to GAPDH, and fold changes were calculated using the $\triangle \triangle C T$ method. Significance was calculated using a One-way ANOVA, and ${ }^{* * *} \mathrm{P}<.005$ and ${ }^{* * * *} \mathrm{P}<.001$.

\section{Immunofluorescence and Confocal Laser Scanning Microscopy}

TIGK cells were grown on Lab-Tek II chamber slides, and after bacterial challenge cells were washed twice with phosphate buffered saline (PBS), fixed and permeabilized with 4\% paraformaldehyde and $0.1 \%$ Triton $\mathrm{x}-100$ for $10 \mathrm{~min}$ at room temperature. Goat serum (10\%) in TBST was used to block the cells for $20 \mathrm{~min}$ at room temperature. Cells were incubated with primary antibody in TBST with $10 \%$ goat serum overnight at $4^{\circ} \mathrm{C}$. Cells were washed 3 times with TBST, then incubated with Alexa Fluor 488 secondary antibody

$(1: 200)$ for $2 \mathrm{~h}$ at room temperature in the dark. After washing as before and blocking with $10 \%$ goat serum, cells were incubated with Texas Red-Phalloidin (1:100) for $2 \mathrm{~h}$ at room temperature in the dark. Cells were washed 3 times, and coverslips were mounted using ProLong Gold (Invitrogen) with 4'6-diamidino-2-phenylindole (DAPI) overnight. Slides were scanned with a Leica SP8 confocal microscope, and images were analyzed with Volocity 6.3 Software (PerkinElmer).

Nuclear localization was quantified using the 3D image processing software IMARIS (Bitplane AG). The Surfaces function in IMARIS was used to create a 3D model of the nuclei from the DAPI channel. IMARIS surfaces then calculated Alexa Fluor 488 voxels within each nucleus, which was normalized to the volume of the nucleus. Total normalized Alexa Fluor 488 was then divided by the number of cells analyzed. 


\section{Transwell Assays}

For studying bacterial interactions with the epithelial cells, $1 \times 10^{5}$ cells were grown only in the lower chamber of a $0.4 \mu \mathrm{m}$ transwell filter plate. Cells were challenged with $P$. gingivalis, and the upper chamber contained cell culture media and either S. gordonii, $S$. mutans, or S. oralis.

For studying epithelial cell to epithelial cell interactions, TIGKs were cultured in both the lower chamber and on the transwell insert in the upper chamber. $P$. gingivalis was added to the upper chamber only.

To measure the motility of TIGK cells we used a matrigel migration assay (BD Biosciences). Cells $\left(2 \times 10^{5}\right)$ cells were seeded onto the matrigel insert in the upper chamber, with cell culture medium in the lower chamber, and cultured for $18 \mathrm{~h}$. The filter was removed, and the surface was scraped with a cotton swab to remove any cells that did not migrate. Cells that migrated through the filter were fixed with $1 \%$ methanol, and stained with toluidine blue. Cells were counted from 3 random fields at 20x using a Nikon Eclipse T100 microscope.

\section{RNAseq}

RNA was extracted using the RNAqueous-Micro Total RNA Isolation kit (ThermoFisher). The TruSeq Stranded Total RNA with RiboZero Plus kit (Illumina) was used to generate a sequencing library from $1 \mu \mathrm{g}$ of total RNA. Paired-end sequencing was performed on an 
Illumina Nextseq 500 at the University of Louisville Core using the Nextseq 500 HighOutput Kit (150 cycles) (Illumina). Base calls were made using the BaseSpace FastQ Version 1.0.0 application (Illumina, Inc.). Raw gene counts with a minimum of two counts per million in at least one sample were used as the initial dataset for downstream RNASeq analyses. These raw counts were used for the determination of differentially expressed genes via the DESeq2 Bioconductor/R package (DOI: 10.18129/B9.bioc.DESeq2) using the recommended guidelines. Output was in the form of log2 fold change expression values and $p$-values adjusted for multiple comparisons using the Benjamini-Hochberg procedure. Differentially expressed genes were defined using cutoffs of 1 for the log 2 fold change and an adjusted p-value of 0.05 . This output was used as input for the generation of volcano plots using the EnhancedVolcano Bioconductor/R package (DOI: 10.18129/B9.bioc.EnhancedVolcano). This output was also used as input for functional enrichment analysis through the String Database version 11 using an FDR stringency of 1 percent and a minimum interaction confidence score of 0.400 for network generation. For principal component analysis (PCA) and heatmap generation, the raw count data were made homoscedastic using a regularized logarithm transformation. PCA was conducted using base R and PCA plots generated using the ggfortify R package. Gene count data for heatmap generation was further converted into z-scores and used as input into the ComplexHeatmap Bioconductor/R package.

\section{Gingipain Activity Assay}


Culture supernatant from $P$. gingivalis was filtered $(0.4 \mu \mathrm{m})$ and combined with filtered supernatant from with either aerobic or anaerobic cultures of S. gordonii or S. mutans. For experiments using $\mathrm{H}_{2} \mathrm{O}_{2}$, culture supernatant from $P$. gingivalis was incubated with $\mathrm{H}_{2} \mathrm{O}_{2}$ for 30 min. Gingipain activity was visualized using the chromogenic substrate LBAPNA. The rate of substrate hydrolysis and the accumulation of $p$-nitroanilide were monitored spectrophotometrically at $405 \mathrm{~nm}$ over time in a Spectramax M5 reader (Molecular Devices).

\section{Statistical Analysis}

Each analysis is representative of at least 3 biological replicates with technical duplicates, and confocal images are representative of 3 biological replicates with at least 3 randomly scanned areas of the chamberslide. ANOVA with Tukey's multiple comparison test were conducted using GraphPad Prism V8. Statistical analyses of RNA-Seq data are described above. 


\section{CHAPTER 3 RESULTS}

To characterize the interplay between $P$. gingivalis and S. gordonii at the epithelial barrier we first employed RNAseq to identify host genes that are differentially regulated in the presence of each organism alone versus the two species present together. Telomerase immortalized gingival keratinocytes (TIGKs) were challenged with either $P$.

gingivalis, S. gordonii, or a dual infection with both organisms. Fig. 5A shows the PCA plot for the RNAseq, where we observed tight clustering of samples within the same group and separation between each of the groups, indicating distinct transcriptional responses. We enriched for genes involved in Notch signaling in Fig. 5B, which was recently shown to be activated by $P$. gingivalis [42]. We found in Fig. 5B that the Notch1 and Notch3 receptors, Jagged1 ligand (Jag1), and downstream targets OLFM4 and Hes5 were all upregulated by $P$. gingivalis.

OLFM4 is an antiapoptotic glycoprotein which promotes tumor growth [43], and is selectively expressed in inflamed epithelium [47]. In the dual infection group, expression of all of these genes was significantly reduced compared $P$. gingivalis alone, and differential expression of the Notch pathway in response to $P$. gingivalis $(\mathrm{Pg})$ did not occur in the presence of S. gordonii (Sg). The results for the Pg vs. NI, Pg+Sg vs. Pg, Pg+Sg vs. Sg, and Pg vs. Sg comparisons from the RNAseq were also summarized as a volcano plot in Fig. 5C-F, and shared differentially expressed genes summarized as upset charts in Fig. 5G-H. The transcriptome data on P. gingivalis induction of OLFM4 was corroborated 
in a dose and time dependent manner at the transcriptional (Fig. 6A) level, and at $24 \mathrm{~h}$ the protein level by Western blotting (Fig. 6B ), ELISA (Fig. 6C) and confocal microscopy (Fig. 6D), all showed an increase in OLFM4 protein in response to $P$. gingivalis. These mRNA and protein analyses identify that $P$. gingivalis-induced upregulation of OLFM4 through Notch signaling is reduced when S. gordonii is present. 
A

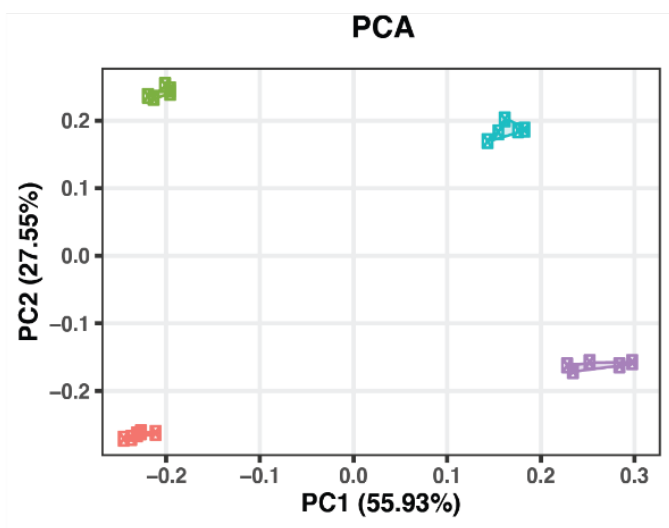

C

Pg vs. NI

Log2FC cutoff $=1$, adj. p-value cutoff $=0.05$
B

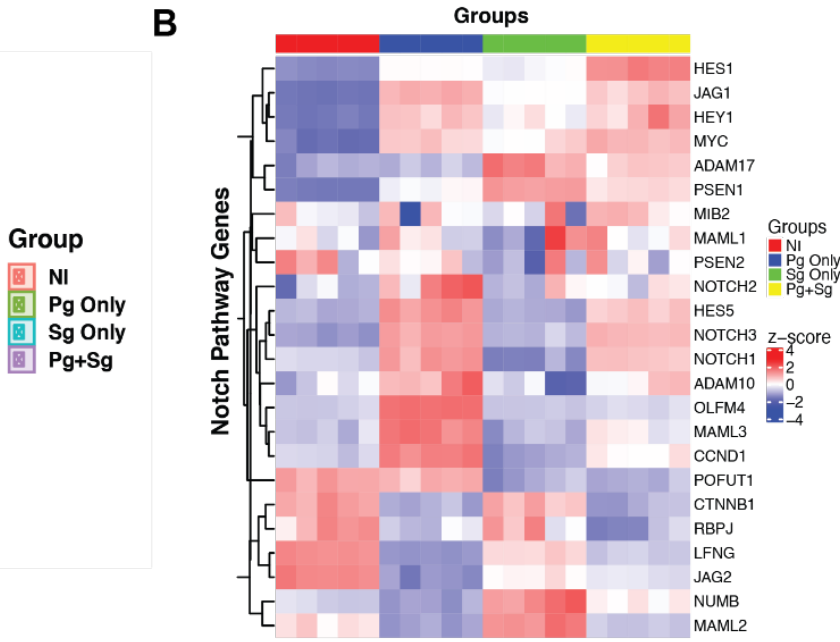

Group

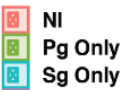

$\mathrm{Pg}+\mathrm{Sg}$

- NS - $\log _{2} F C \bullet p$-value $\bullet p$-value and $\log _{2} F C$

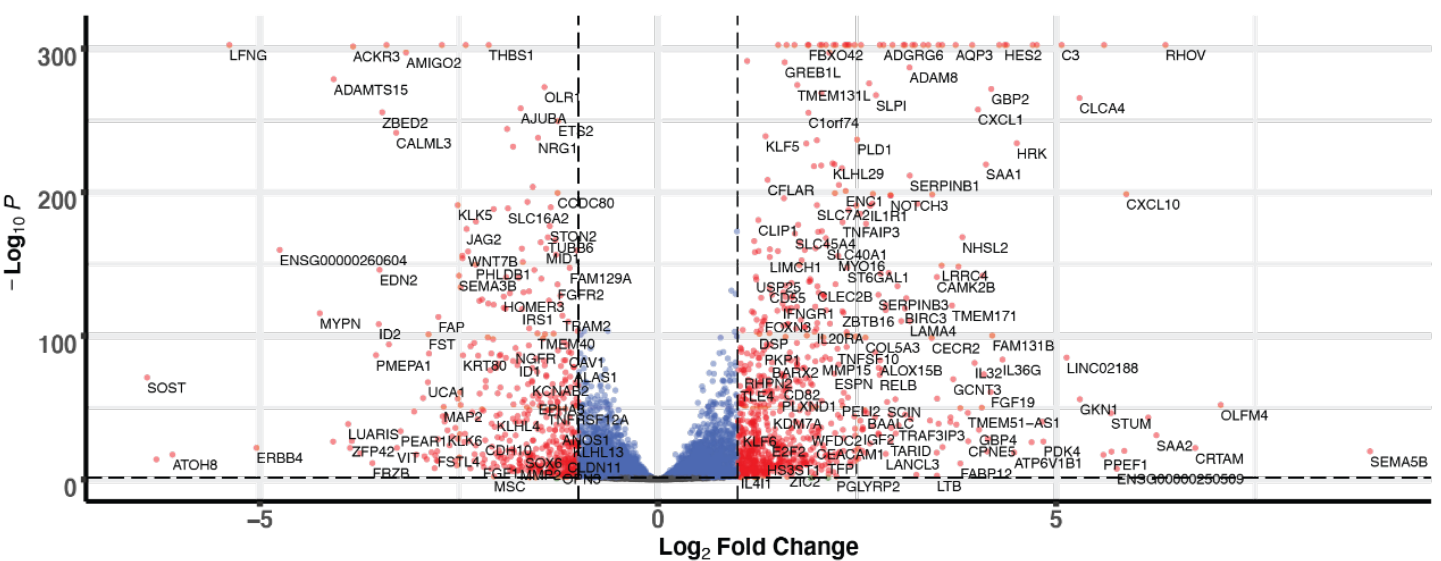

D

Total $=14607$ input genes

$\mathrm{Pg}+\mathrm{Sg}$ vs $\mathrm{Pg}$

Log2FC cutoff $=1$, adj. $p$-value cutoff $=0.05$

- NS - p-value - p-value and $\log _{2}$ FC

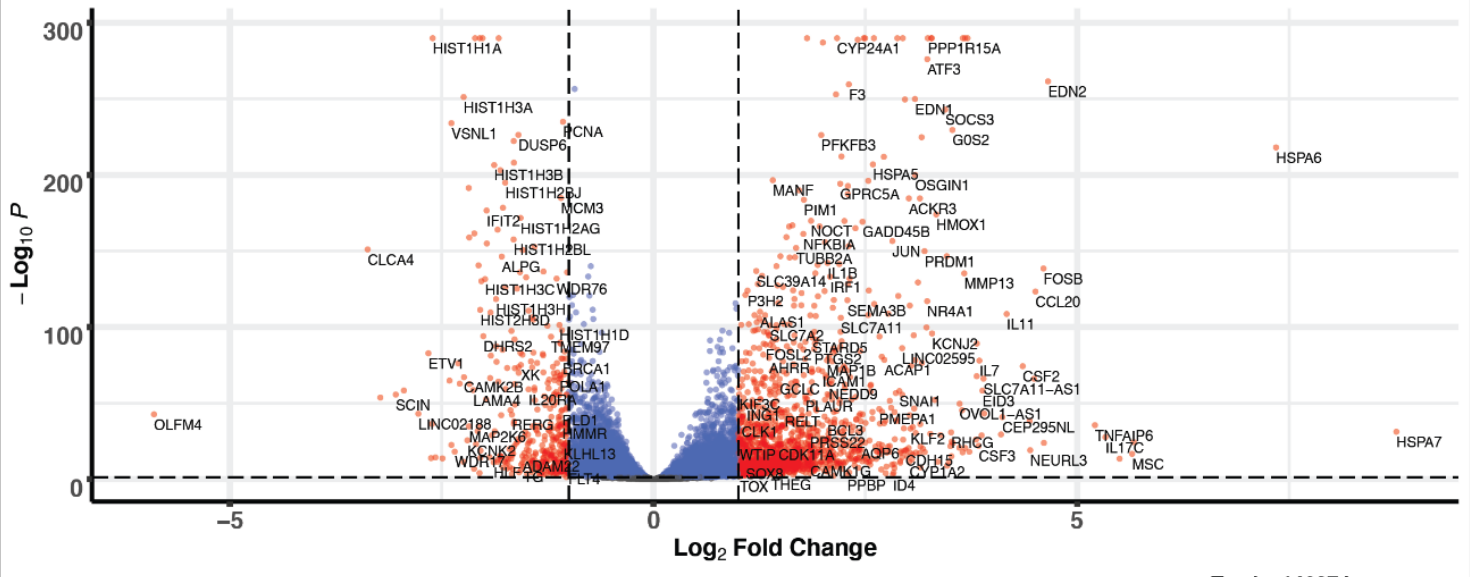

Total $=14607$ input genes 
E

$\mathrm{Pg}+\mathrm{Sg}$ vs $\mathrm{Sg}$

Log2FC cutoff $=1$, adj. p-value cutoff $=0.05$

- NS • $\log _{2} \mathrm{FC} \bullet \mathrm{p}$-value • p-value and $\log _{2} \mathrm{FC}$

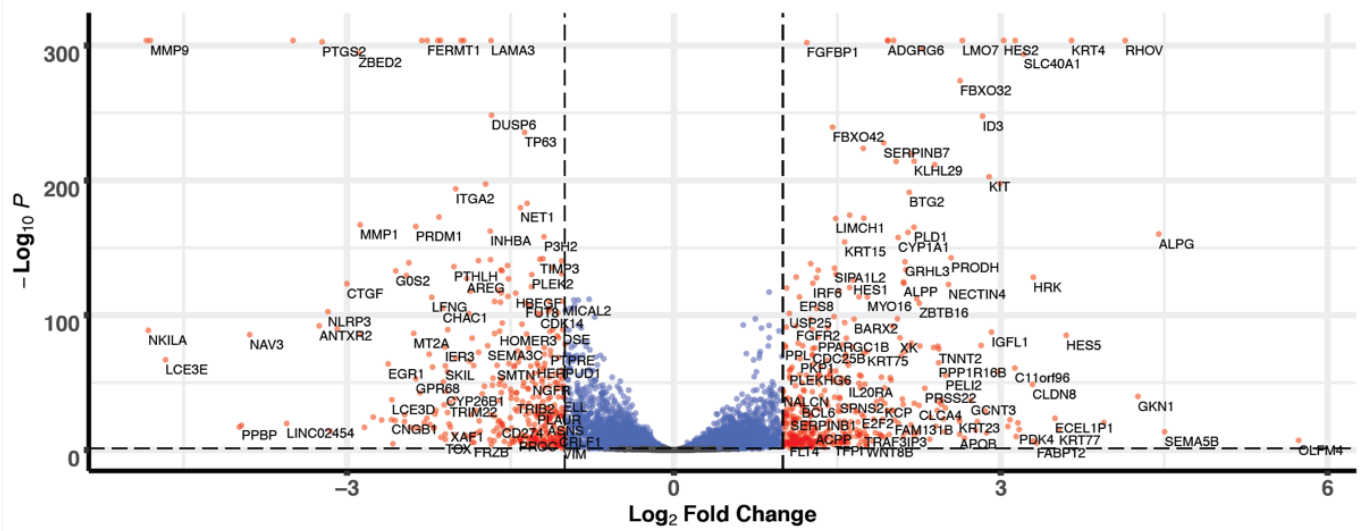

$\mathbf{F}$

Pg vs Sg

Log2FC cutoff $=1$, adj. $p$-value cutoff $=0.05$

Total $=14607$ input genes

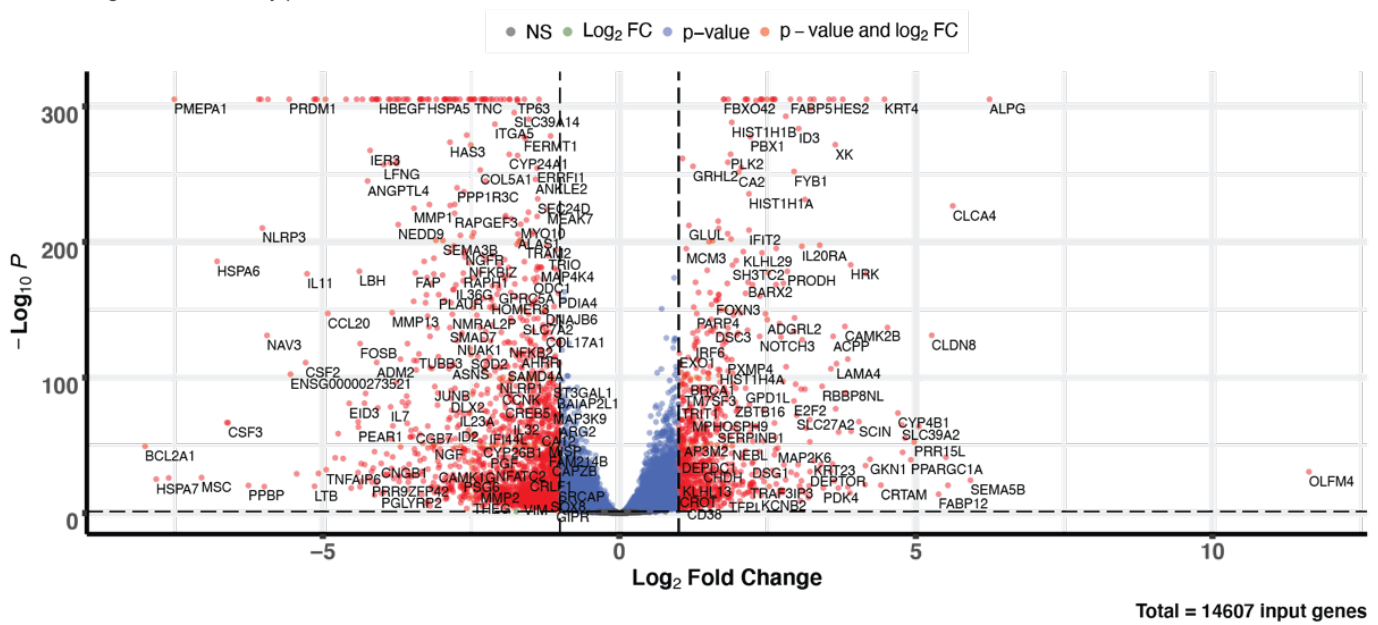

G

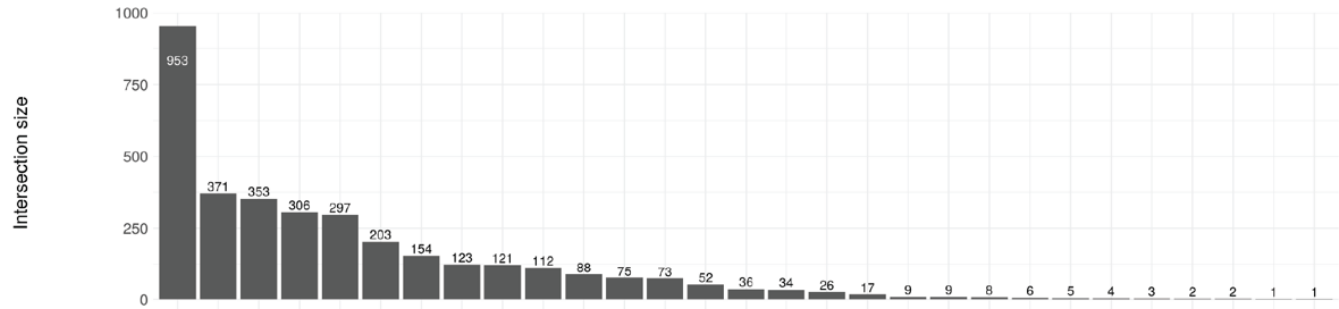

TIGK - Upregulated DEGs

FC cutoff $=2$, adj. $\mathrm{p}$-value cutoff $=0.05$

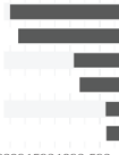

PgSg_vs_NI_Up • • • • ० ०

Sg_vs_NI_Up -

Pg_vs_Sg_Up

Pg_vs_PgSg_Up

$\mathrm{Sg}$ vs PgSg Up

Set size

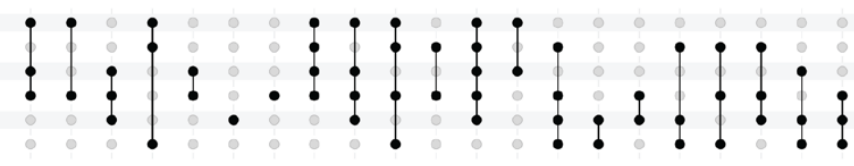

Comparisons 
H

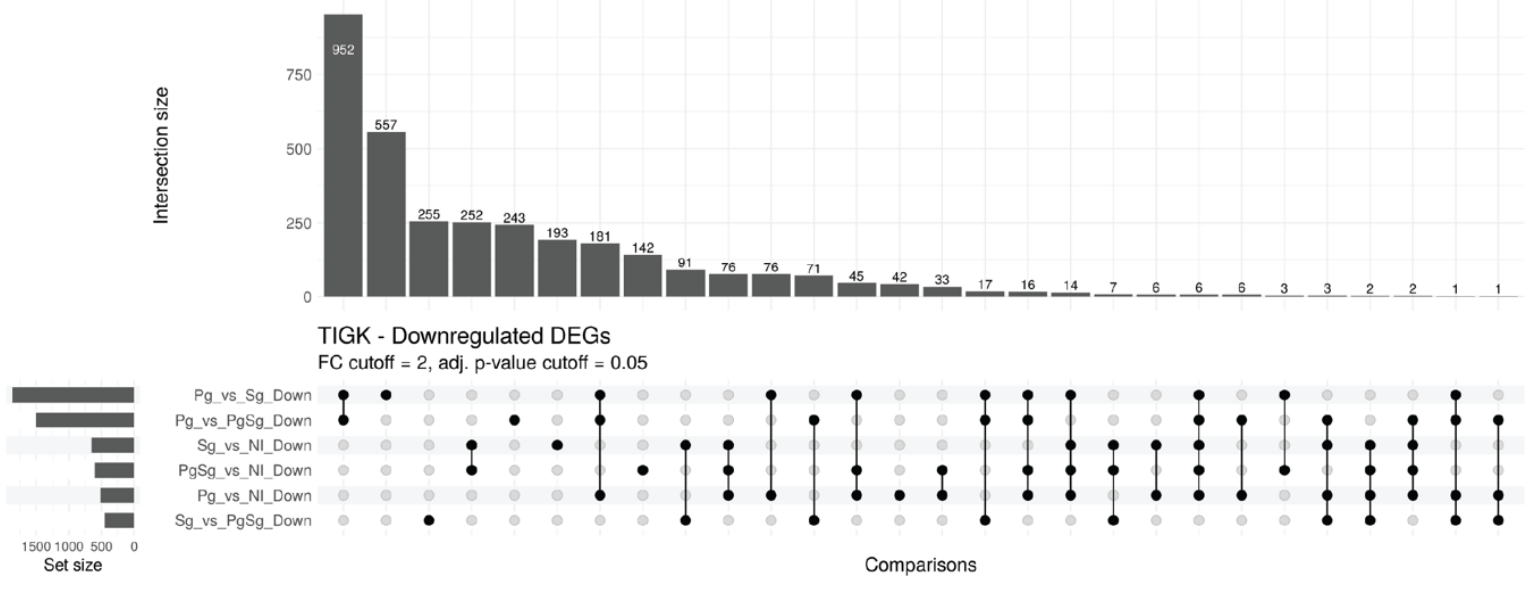

Figure 5: RNAseq of TIGK cells challenged with $P$. gingivalis and/or $S$. gordonii TIGK cells were challenged with $P$. gingivalis (MOI 100) and/or S. gordonii (MOI 50) for $1 \mathrm{~h}$, then the media was changed to remove any non-adherent bacteria. Cells were harvested at $24 \mathrm{~h}$, at which point RNA was harvested, and libraries were prepared for RNAseq. The PCA plot (A) shows distinct separation of non-infected (NI), P. gingivalis (Pg), S. gordonii $(\mathrm{Sg})$, and $P$. gingivalis co-challenged with $S$. gordonii $(\mathrm{Pg}+\mathrm{Sg})$ groups, as well as a tight clustering of individual samples within each group. Notch signaling is transcriptionally regulated by $P$. gingivalis, and $S$. gordonii interrupts activation by $P$. gingivalis, specifically reducing Notch1, Notch3, Jagged1, Hes5, and OLFM4 (B). Graphical comparisons of gene expression are represented by volcano plots for $P$. gingivalis vs. NI (C), P. gingivalis $+S$. gordonii vs. $P$. gingivalis alone (D), $P$. gingivalis $+S$. gordonii vs. S. gordonii alone (E), and $P$. gingivalis alone vs. S. gordonii alone (F). A summary of the shared upregulated (G) and downregulated $(\mathrm{H})$ genes for each comparison have been presented as upset charts. 
A

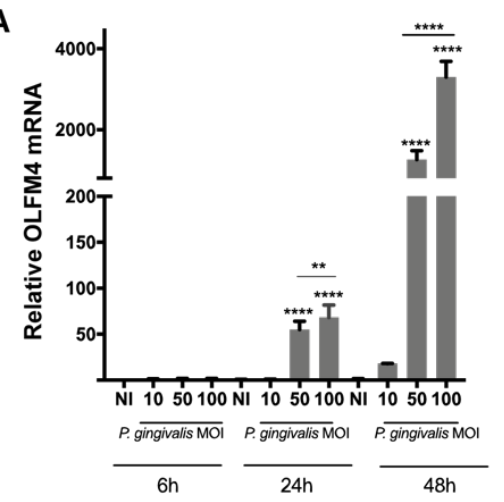

D

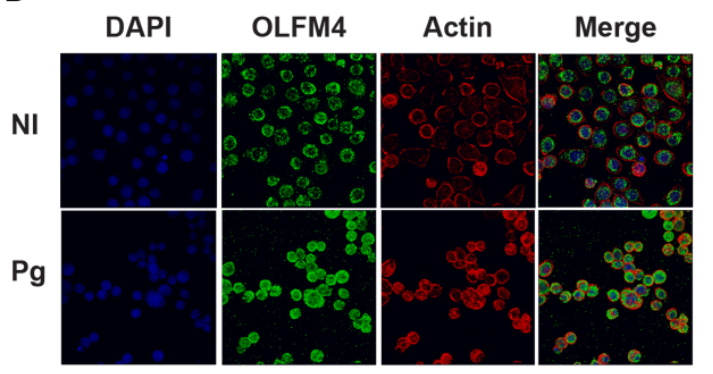

F

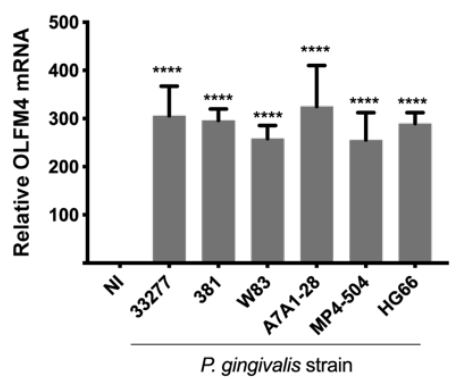

I

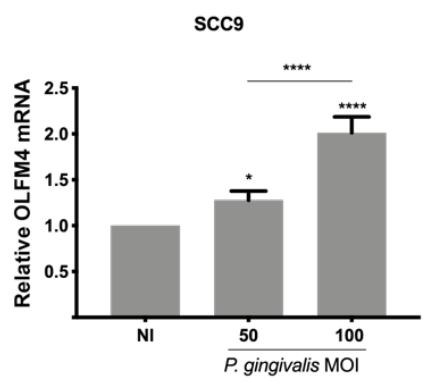

B
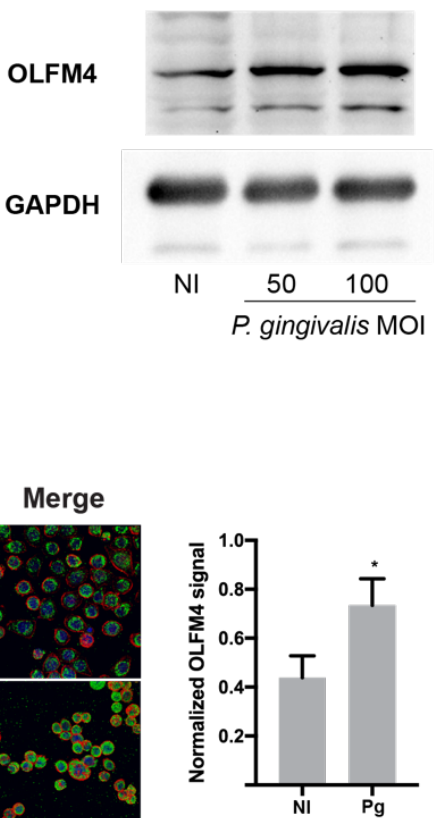

G

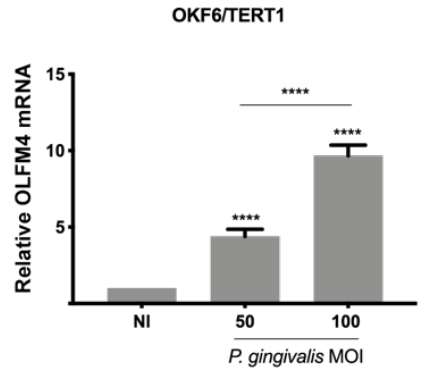

C

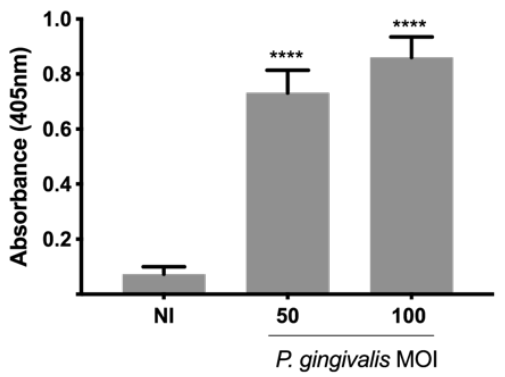

E

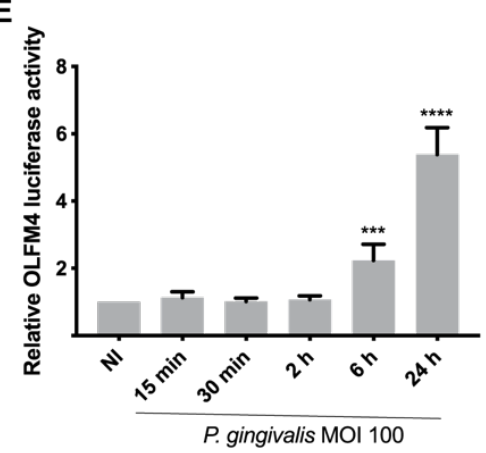

H

ESCC9706

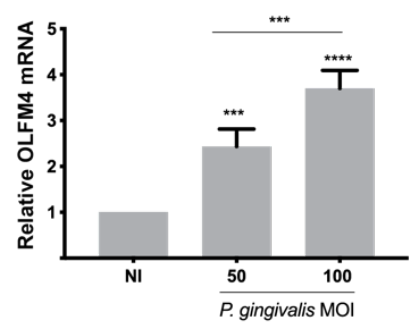


Figure 6: Regulation of OLFM4 by $P$. gingivalis

TIGK cells were challenged with $P$. gingivalis ATCC 33277 at an $\mathrm{MOI}$ of either 10, 50, or 100 for $6 \mathrm{~h}, 24 \mathrm{~h}$, or $48 \mathrm{~h}$, then RNA was harvested and reverse transcribed into cDNA for qRT-PCR (A). OLFM4 upregulation was first observed at $24 \mathrm{~h}$, and significantly increases in a dose and time dependent manner. Cells were harvested as previously described at $24 \mathrm{~h}$, and western blot confirmed increases OLFM4 protein expression (B). As OLFM4 is a secreted protein, verification of increased secretion was investigated by ELISA of cell culture supernatants. Cells were challenged with $P$. gingivalis at indicated MOls, then supernatants were collected, filtered, and concentrated (C). Cells were grown on chamber slides and challenged for $24 \mathrm{~h}$ at an MOI 100, then probed with OLFM4 antibodies (green), phalloidin labeling actin (red), and lastly the nucleus was stained with DAPI (blue). $P$. gingivalis significantly increased OLFM4 intensity, which was normalized to the size of each nucleus (D). 500 bp upstream of OLFM4 was cloned into the promoterless PGL3Firefly luciferase construct, and was used to measure promoter activity of TIGK cells challenged with $P$. gingivalis at an $\mathrm{MOI}$ of 100 for the indicated times. $P$. gingivalis increases promoter activity at $6 \mathrm{~h}$, with the greatest increase at $24 \mathrm{~h}(\mathrm{E})$. Multiple strains of $P$. gingivalis were tested at an $\mathrm{MOI}$ of 100 for $24 \mathrm{~h}$, including the encapsulated W83 and low passage clinical isolate MP4-504 (F). Each strain was equally able to stimulate OLFM4 expression. $P$. gingivalis upregulated OLFM4 in OKF6/TERT1 cells, which are immortalized non-cancerous mucosal cells (G), as well as the esophageal cancer derived cell line ESCC9706 $(\mathrm{H})$ and tongue squamous cell carcinoma derived SCC9 cell line (I). Target genes for qRT-PCR were normalized to GAPDH, and fold changes were calculated using the $\triangle \triangle C T$ method. Significance was calculated using a One-way ANOVA, and ${ }^{*} P<.05$, $* * \mathrm{P}<.01, * * * \mathrm{P}<.005$, and $* * * * \mathrm{P}<.001$.

To confirm regulation of OLFM4 at the transcriptional level, TIGKs were transfected with a luciferase-reporter construct containing the regulatory fragment spanning 500bp upstream of the coding region [126]. In a luciferase assay challenge with P. gingivalis increased OLFM4 promoter activity beginning at $6 \mathrm{~h}$ (Fig. 6E). We explored multiple $P$. gingivalis strains to verify upregulation of OLFM4 is a property conserved across the species. In Fig. 6F we show all tested strains of $P$. gingivalis induced elevated OLFM4 expression, including the fimbriated/non-encapsulated lineage (33277, 381, and the gingipain hypersecreting HG66), the encapsulated/afimbrial lineage (W83), the fimbriated/encapsulated lineage (A7A128) and the low-passage clinical isolate MP4-504 
[127-130]. Next, we determined whether OLFM4 regulation by $P$. gingivalis is specific to gingival epithelial cells. The OKF6 line are telomerase immortalized buccal mucosa cells, and we found that $P$. gingivalis upregulates OLFM4 in these cells, albeit to lower levels than occur in TIGKs in Fig. 6G. Tongue (SCC9) and esophageal (ESCC9706) squamous cell carcinoma cells also displayed an increase in OLFM4 expression in response to $P$. gingivalis challenge, again at a substantially lesser magnitude compared to TIGK cells in Fig. $6 \mathrm{H}-\mathrm{J}$. These results indicate that epithelial cells of the gingiva, which is the primary in vivo habitat of $P$. gingivalis, are more responsive to challenge with the organism, at least in terms of OLFM4 regulation, as compared to cells derived from other sites in the oral and esophageal regions.

Because genes involved in Notch signaling and OLMF4 were coordinately upregulated by $P$. gingivalis exposure, and normalized when S. gordonii was present, we further explored the role of Notch signaling in the regulation of OLFM4 in TIGK cells. There are four Notch receptors which initiate signaling through the pathway, and quantitative (q) RT-PCR showed that expression of Notch1 and Notch3 is enhanced by infection with P. gingivalis in Fig. 7A. To elucidate the role of these Notch receptors in OLFM4 upregulation, expression of each receptor was suppressed using siRNA. Knock down of Notch1 led to significantly decreased OLFM4 mRNA levels in the $P$. gingivalis challenged cells in Fig. 7B, indicative of a role for Notch1 in the signaling pathway exploited by $P$. gingivalis to regulate OLFM4. Knock down of Notch 2 and 4 had no effect on OLFM4 expression, whereas knock down Notch3 significantly increased the level of OLFM4, possibly due to increased availability or expression of Notch1. Notch signaling is activated 
by the ligands Jag1, Jag2, DLL1, DLL3, and DLL4, and by qRT-PCR $P$. gingivalis upregulates expression of Jag1 and downregulates Jag2 in Fig. 7C. As shown in Fig. 7D knock down of Jag1 led to decreased induction of OLFM4 by $P$. gingivalis, whereas knocking down Jag2 increased OLFM4, indicating that Jag2 expression may interfere with signaling through Notch1. Suppression of DLL 1, 3, or 4 did not negatively influence OLFM4 mRNA levels in response to $P$. gingivalis. Knock down of Notch1 and Jag1 also diminished $P$. gingivalisdependent regulation of an additional target of the Notch signaling pathways, Hes5 (Fig. 7E),. Collectively these data corroborate the importance of the Jag1-Notch1 signaling pathway in TIGK responses to $P$. gingivalis. 
A

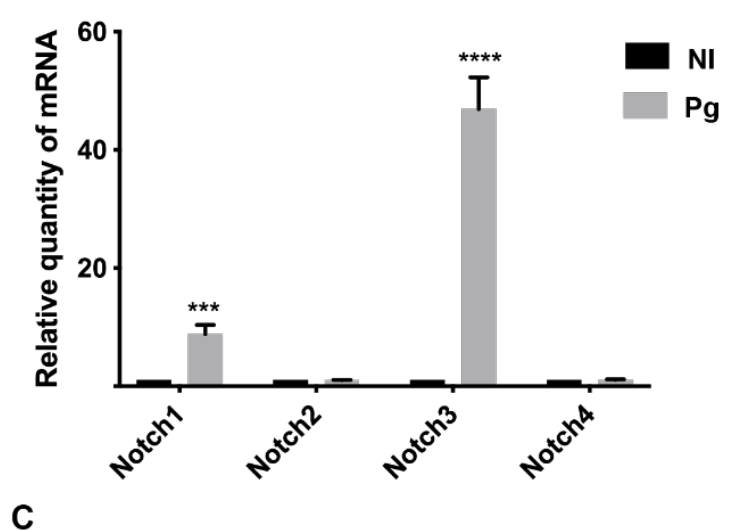

C

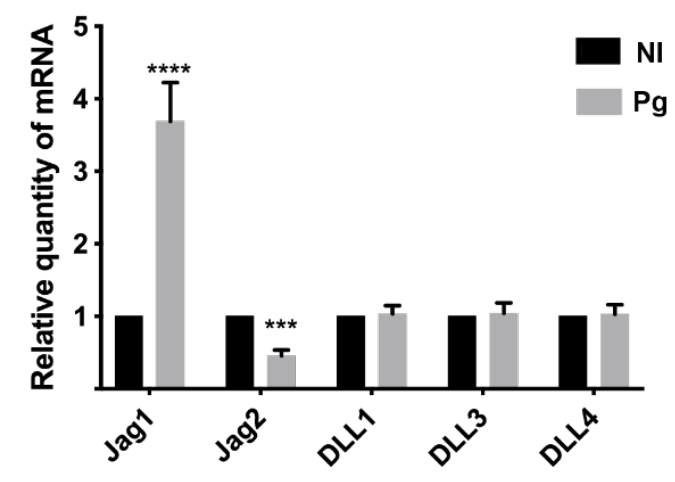

E

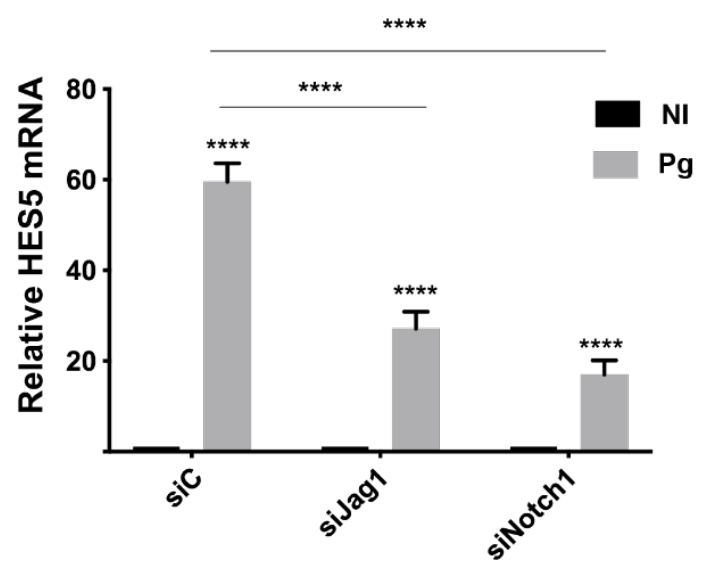

B
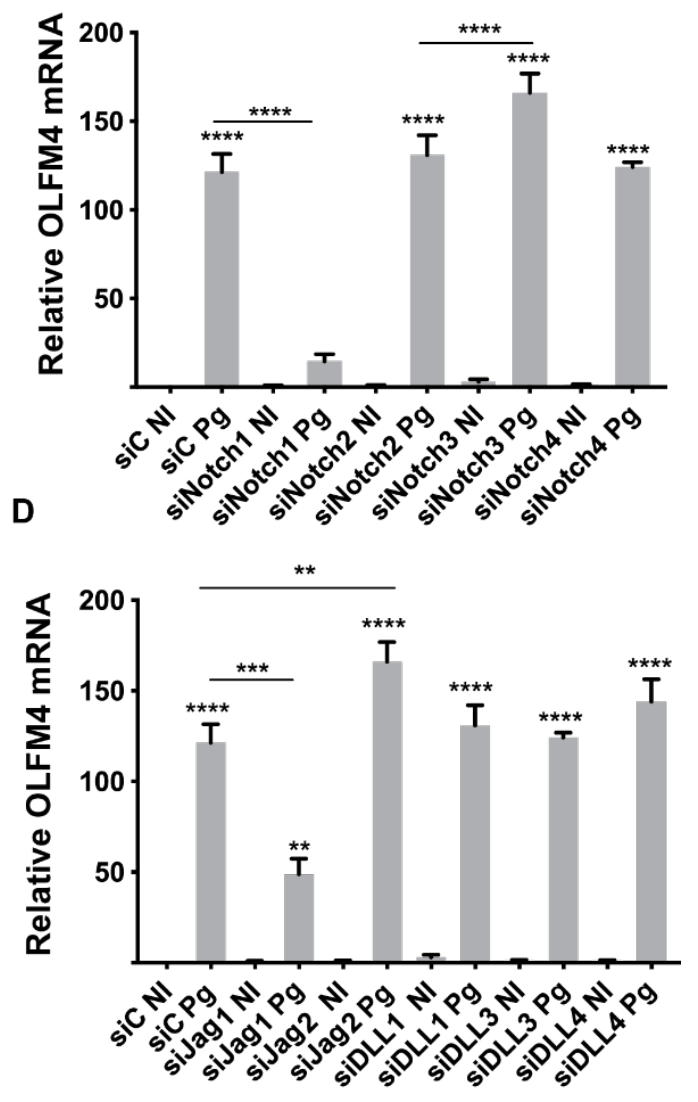

Figure 7: Regulation of Notch signaling by $P$. gingivalis

$P$. gingivalis upregulates Notch1 and Notch3 receptors, but not Notch2 and Notch4 at MOI 100 for $24 \mathrm{~h}$ in TIGK cells by qRT-PCR (A). Notch1-4 receptors were individually knocked down through siRNA transfection, and scrambled siRNA was used as a control. $P$. gingivalis requires Notch1, but not Notch2-4 for OLFM4 upregulation (B). Similarly, we investigated regulation of Notch ligands Jag1, Jag2, DLL1, DLL3, and DLL4 by $P$. gingivalis as in $(A)$ and found $P$. gingivalis significantly upregulates Jag1, downregulates Jag2, and does not regulate DLL1, DLL3, and DLL4 (C). Employing siRNA transfections as in (B) for Notch ligands we found that Jagged1 was required for OLFM4 upregulation by $P$. 
gingivalis, but not Jag2, DLL1, DLL3, or DLL4 (D). HES5 is also a downstream target of Notch signaling, and in a similar fashion to OLFM4 requires Notch1 and Jag1 for optimal regulation of HES5 mRNA (E). Target genes for qRT-PCR were normalized to GAPDH, and fold changes were calculated using the $\triangle \triangle C T$ method. Significance was calculated using a One-way ANOVA, and ** $\mathrm{P}<.01, * * * \mathrm{P}<.005$, and **** $\mathrm{P}<.001$.

It has been shown that endothelial cells are able to cleave Jag1 and release a soluble form of Jag1 that can then bind to Notch receptors on colorectal cancer cells and activate signaling [131]. To explore whether a similar mechanism of activation occurs in our model, we utilized a transwell system to determine whether challenge with $P$. gingivalis causes release of soluble signaling factors. TIGKs were cultured both on the transwell filter in the upper chamber, as well as in the lower chamber. When cells in the upper chamber were challenged with $P$. gingivalis, OLFM4 transcription was upregulated in the lower chamber cells in Figure 8.

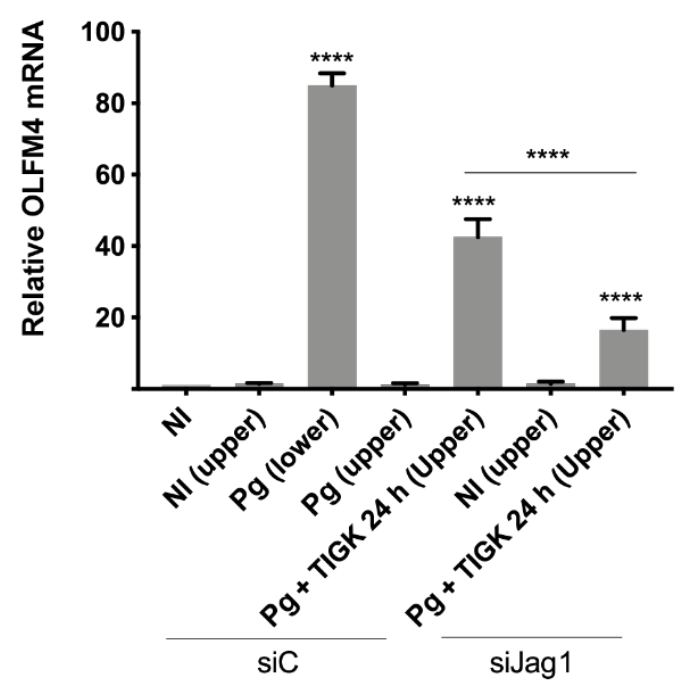

Figure 8: $P$. gingivalis induces paracrine activation of Notch signaling through soluble Jagged1

TIGK cells were grown in the lower chamber of a 0.4um transwell plate, as well as on top of the transwell filter. Only cells above the transwell filter were transfected with either 
control siRNA or siJag1. Cells were then challenged with $P$. gingivalis either above or below the transwell filter as indicated, and only cells below the transwell filter were harvested for RNA and reverse transcribed for qRT-PCR. $P$. gingivalis alone was not enough to stimulate the lower TIGK cells in the upper chamber of the transwell filter, but when TIGK cells were seeded onto the upper chamber activation of OLFM4 in the lower chamber was maintained. Additionally, specifically knocking down Jag1 reduced OLFM4 activation in the lower chamber cells, which implicates a soluble form of Jag1 being involved in this paracrine activation mechanism. Target genes for qRT-PCR were normalized to GAPDH, and fold changes were calculated using the $\triangle \triangle C T$ method. Significance was calculated using a One-way ANOVA, and **** $\mathrm{P}<.001$.

To evaluate the role of Jag1 in this regulation Jag1 was knocked down by siRNA in cells in the upper chamber. After challenge of upper chamber cells with $P$. gingivalis there was significantly less OLFM4 expression in the lower chamber cells compared to the siControl condition as shown in Figure 8. These results indicate that $P$. gingivalis can activate the Notch pathway in a paracrine manner by increasing production of Jag1.

OLFM4 is also a target gene of the Wnt/ $\beta$-catenin pathway and of the NF-KB transcription factor [126]. As $P$. gingivalis can activate $W n t / \beta$-catenin signaling, but not NF-KB $[32,57]$, we thus questioned whether the Wnt/ $\beta$-catenin pathway is also involved in P. gingivalis-induced expression of OLFM4. However, neither pharmacological inhibition of Wnt nor siRNA knockdown of $\beta$-catenin had an affect on OLFM4 induction in response to $P$. gingivalis as shown in Fig. 9 A-B. 
A

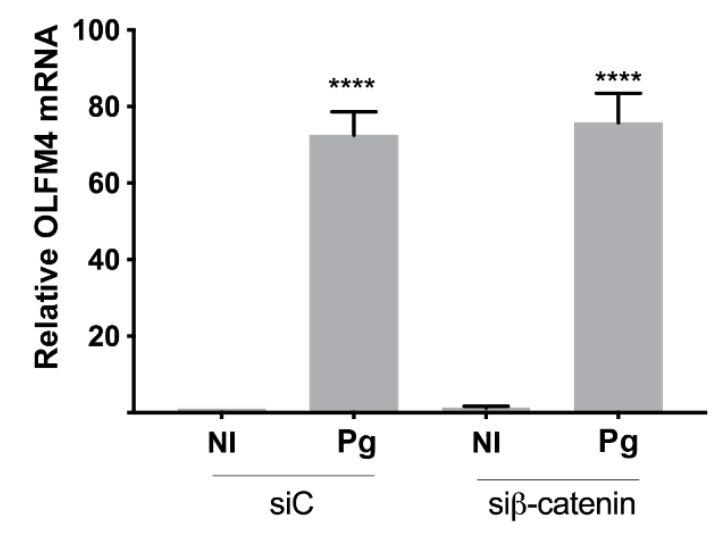

C

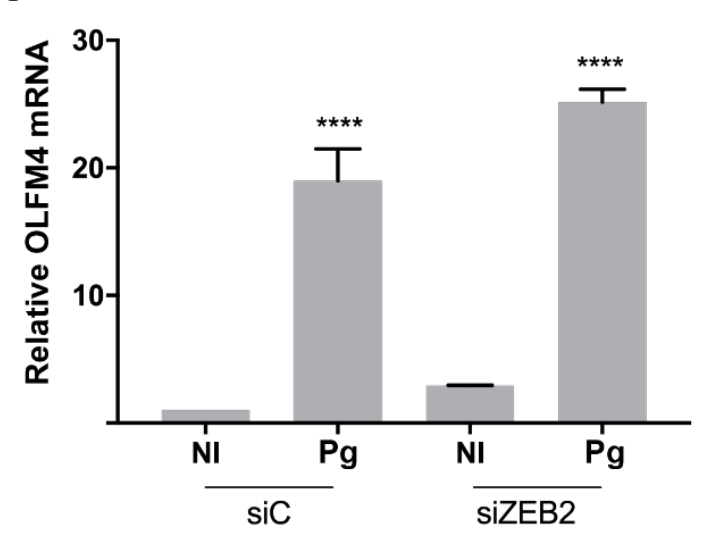

B

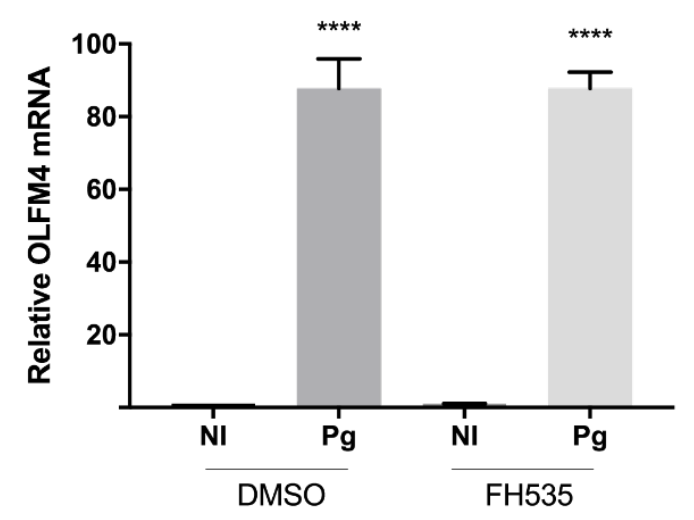

Figure 9: Wnt/ $\beta$-catenin and ZEB2 are not required for activation of OLFM4 by $P$. gingivalis $P$. gingivalis activates $W n t / B$-catenin signaling, and this pathway has been implicated in OLFM4 regulation. We utilized siRNA targeting $\beta$-catenin $(A)$ and a pharmacological inhibitor of Wnt signaling, FH535 (B) in TIGK cells. Cells were then challenged with $P$. gingivalis at an MOI of 100 for $24 \mathrm{~h}$, and RNA was harvested for QRT-PCR. $\beta$-catenin knock and pharmacological inhibition of Wnt signaling did not impede the regulation of OLFM4. $P$. gingivalis also activates the epithelial to mesenchymal transition transcription factor ZEB2, which could potentially bind the promoter region based on a consensus sequence found upstream of the coding region of OLFM4. Knocking down ZEB2 did not prevent regulation of OLFM4 by $P$. gingivalis $(C)$. Target genes for qRT-PCR were normalized to $\mathrm{GAPDH}$, and fold changes were calculated using the $\triangle \triangle \mathrm{CT}$ method. Significance was calculated using a One-way ANOVA, **** $\mathrm{P}<.001$. 
In silico interrogation of the promoter region of OLFM4 using the eukaryotic promoter database, revealed the presence of a Zeb2 consensus binding element. Zeb2 activity is enhanced by $P$. gingivalis, hence we examined its potential involvement in OLFM4 regulation. As shown in Fig. 9C, siRNA knock down of Zeb2 did not prevent increased OLFM4 mRNA production in response to $P$. gingivalis. This finding further supports the importance of Notch signaling in the regulation of OLFM4 by P. gingivalis.

One mechanism for transcriptional regulation of Jag1 is through the integration of Notch and TGF- $\beta$ signaling. SMAD3, a component of the TGF- $\beta$ transcriptional activation complex, has been shown to activate Notch signaling through upregulation of Jag1 and Hey1 [132]. We show in Fig. 10 that SMAD3 is involved in P. gingivalis-induced Jag1 transcriptional regulation.

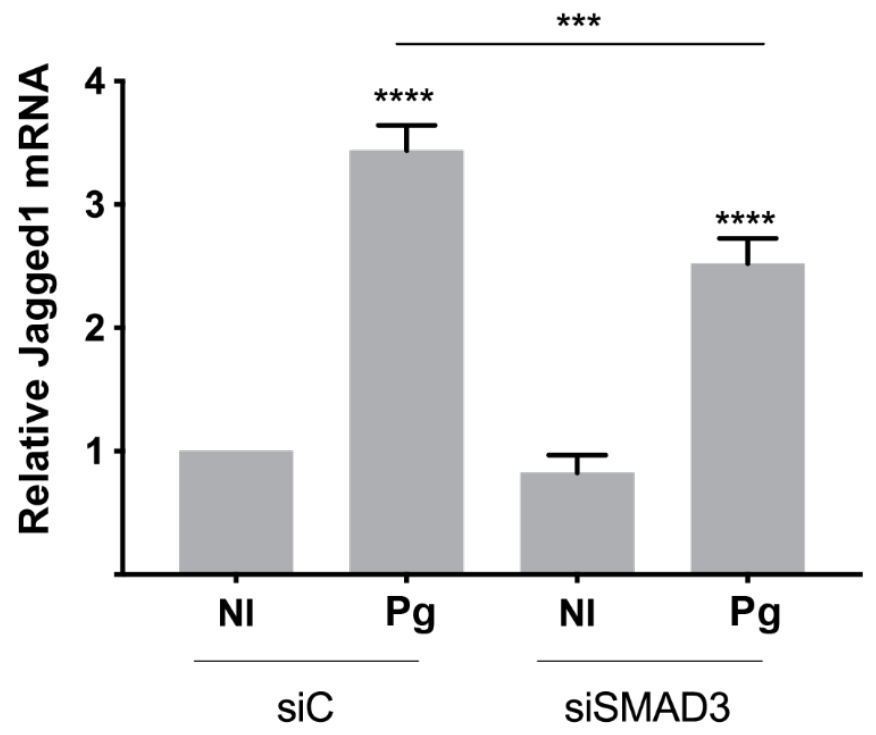

Figure 10: SMAD3 enhances upregulation of Jagged1 by $P$. gingivalis 
TGF- $\beta$ signaling intersect at the SMAD3-Jag1 signaling axis. SMAD3 has been shown to transcriptionally regulate Jag1, leading to activation of Notch signaling. Here we knocked down SMAD3 with siRNA in TIGK cells, and used scrambled siRNA as a control. TIGK cells were then challenged with $P$. gingivalis for $24 \mathrm{~h}$, and RNA was harvested for qRT-PCR. SMAD3 knockdown significantly reduced Jag 1 transcript, which implicates TGF- $\beta$ signaling as a potential activator of Notch signaling. SMAD3 transcript was normalized to GAPDH, and fold changes were calculated using the $\triangle \triangle C T$ method. Significance was calculated using a One-way ANOVA, *** $\mathrm{P}<.005$, and $* * * * \mathrm{P}<.001$.

To identify the effector molecules of $P$. gingivalis responsible for activating Notch signaling, we examined a panel of strains with mutations in established virulence or colonization factors. As shown in Fig. 11A, loss of fimbrial adhesins or of serine and tyrosine phosphatases did not impact the ability of $P$. gingivalis to regulate OLFM4. In contrast, the $\triangle \operatorname{rgp} A B \Delta k g p$ mutant, which is deficient in the production of the arginine specific (RgpA and $\operatorname{RgpB}$ ) and lysine specific (Kgp) gingipain proteases, was unable to stimulate OFLM4 production. 
A

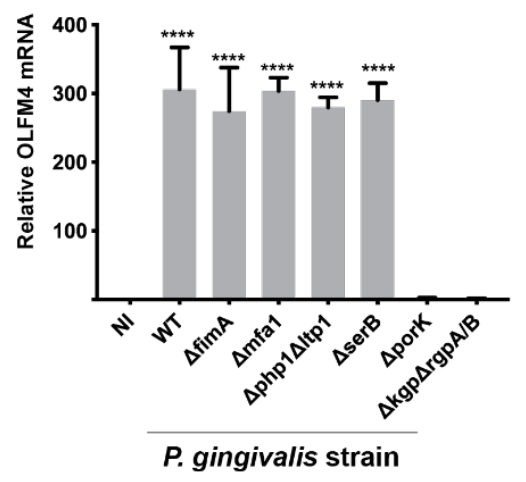

D

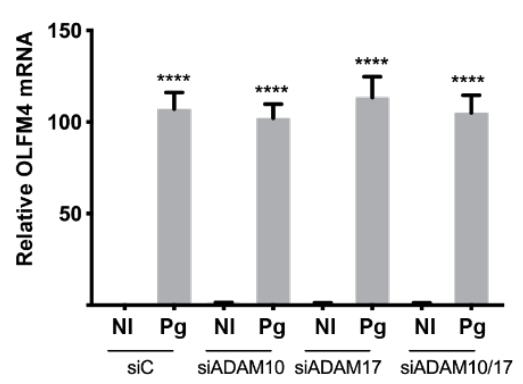

B

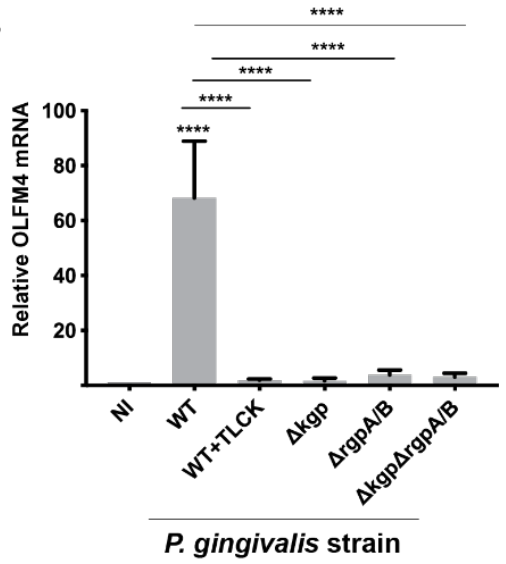

E

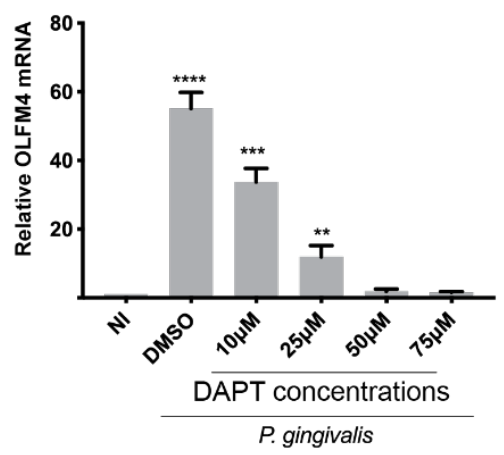

C

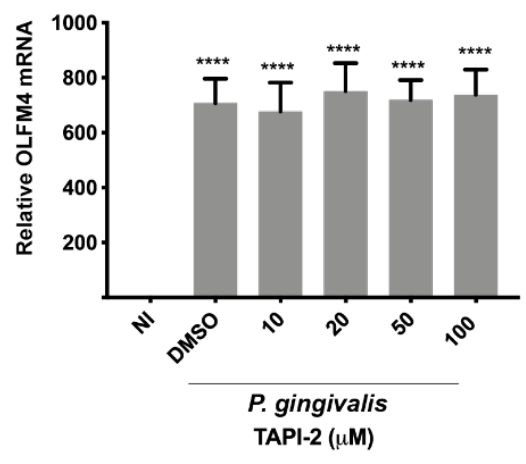

Figure 11: Activation of Notch signaling by $P$. gingivalis gingipains

TIGK cells were challenged for $24 \mathrm{~h}$ at an MOI of 100 with ATCC 33277 wildtype (WT), as well as deletional mutants of adhesins ( $m f a 1, f i m A)$, tyrosine and serine phosphatase network (Itp1/php1, serB), and type 9 secretion system (T9SS) machinery and effectors (porK, $\mathrm{kgp} / \mathrm{rgpA} / \mathrm{rgpB}$ ). Loss of $P$. gingivalis adhesins or phosphatases did not interfere with regulation of OLFM4, but loss of T9SS regulatory protein PorK and T9SS cargo $\mathrm{kgp} / \mathrm{rgpA} / \mathrm{rgpB}$ significantly reduced levels of OLFM4 (A). We then challenged TIGK cells with $P$. gingivalis wildtype treated with the gingipain inhibitor TLCK, as $\mathrm{kgp}, \operatorname{rgp} A / B$, and $\mathrm{kgp} / \mathrm{rgpA} / \mathrm{rgpB}$ deletional mutants (B). RNA was harvested, and OLFM4 transcript measured by qRT-PCR. TLCK treated $P$. gingivalis was unable to stimulate OLFM4 transcription, and an unimpaired proteolytic load was required for OLFM4 regulation. The Notch receptors are sequentially cleaved after binding Notch ligands first by extracellular ADAM10/17 proteases, then by intracellular gamma-secretase. TIGK cells were treated with a pharmacological inhibitor of the extracellular ADAM10/17 proteases TAPI-2 at indicated concentrations, then challenged with $P$. gingivalis for $24 \mathrm{~h}$ (C). Pharmacological inhibition of ADAM10/17 (C) nor siRNA knockdown of ADAM10 and/or ADAM17 (D) impeded $P$. gingivalis induced $O L F M 4$ by qRT-PCR, implicating $P$. gingivalis produced extracellular gingipain proteases in activation of Notch signaling. $P$. gingivalis can also invade epithelial cells, so we also investigated the potential role of intracellular gingipains in cleavage of intracellular Notch. Gamma-secretase inhibitor DAPT was used at indicated concentrations, and in a dose dependent manner reduced $P$. gingivalis induction of 
OLFM4 in TIGK cells (E). This excludes the role of gingipains involvement of Notch activation in an intracellular capacity. Target genes for qRT-PCR were normalized to GAPDH, and fold changes were calculated using the $\triangle \triangle C T$ method. Significance was calculated using a One-way ANOVA, ** $\mathrm{P}<.01, * * * \mathrm{P}<.005$, and $* * * * \mathrm{P}<.001$.

Gingipains are secreted through the type IX secretion system, and loss of Pork, which is required for formation of a functional secretion pore [133] also abrogated the ability of $P$. gingivalis to enhance OLFM4 transcription. We further investigated the role of gingipains by challenging cells with individual gingipain mutants, which revealed that loss of either Kgp or RgpA/B was sufficient to prevent upregulation of OLFM4 (Fig. 11B). Additionally, pretreatment of parental $P$. gingivalis with the gingipain inhibitor TLCK prevented OLFM4 synthesis. Engagement of the Notch receptor by Jag1 induces a conformational change in the Notch which exposes an extracellular region of that can be cleaved by the extracellular proteases ADAM10 and ADAM17 [134]. On the basis of results with gingipains, we hypothesized that these proteolytic enzymes of $P$. gingivalis activate signaling through cleavage of the extracellular domain of Notch. To test this notion, we used TAPI-2, a pharmacological inhibitor of the ADAM10 and ADAM17 proteases [135]. We found that $P$. gingivalis remained capable of activating Notch in the presence of TAPI-2 as shown in Fig. 11C, suggesting that the gingipains can functionally compensate for the loss of the ADAM10 and ADAM17 proteases. This was corroborated by siRNA targeting ADAM10 and ADAM17, which also failed to prevent activation of Notch and upregulation of OLFM4 (Fig. 11D). In contrast, DAPT, which is an inhibitor of gamma secretase, the enzyme that cleaves the intracellular domain of the Notch receptors, completely inhibited stimulation of OLFM4 and HES5 transcription by $P$. gingivalis (Fig. 
11E). Thus, the gingipains are unable to complement cleavage of the intracellular domain of Notch, and function extracellularly to activate Notch. Although $P$. gingivalis can internalize and survive inside gingival epithelial cells [136], production of the gingipains is downregulated intracellularly [137] and may be insufficient function on a membrane protein. Collectively, the results show that $P$. gingivalis can activate Notch1 signaling in a two-hit mechanism, by proteolytic cleavage of the extracellular domain and by increasing production of the Jag1 agonist.

The ecosystem of the oral cavity in which $P$. gingivalis resides is a complex because multispecies community partners engage $P$. gingivalis in multilevel interspecies interactions, which can be either synergistic or antagonistic [102]. Hence, we next investigated the impact of several community partners of $P$. gingivalis on OLFM4 regulation. Co-infection of $P$. gingivalis with either Filifactor alocis, Fusobacterium nucleatum, or Treponema denticola, had no influence on regulation of OLFM4 by $P$. gingivalis (Fig. 12A). 
A

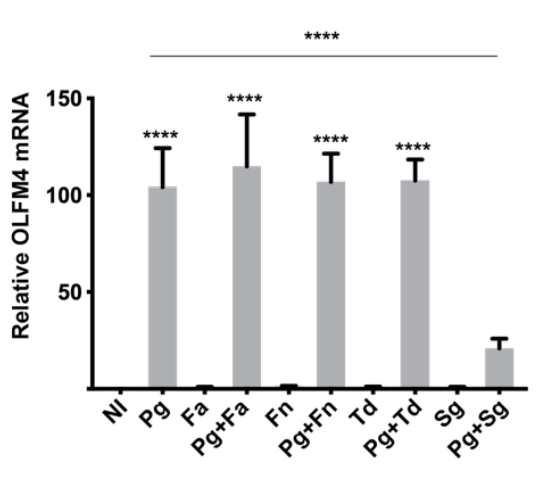

D

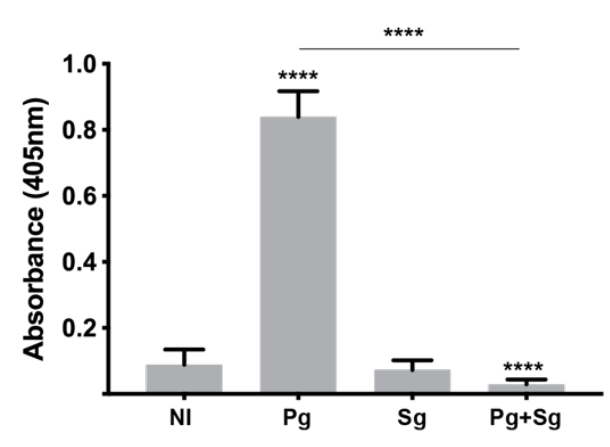

$\mathbf{F}$

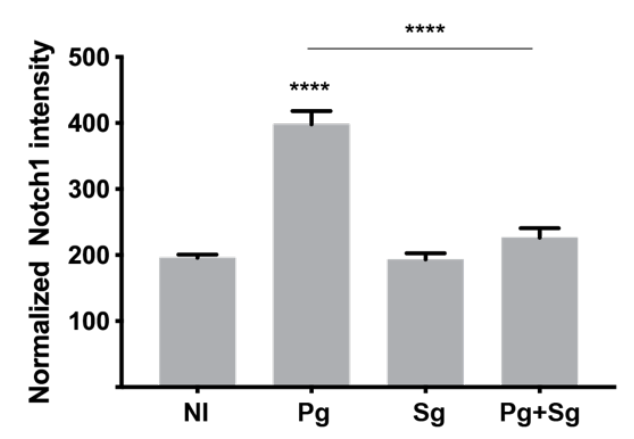

B

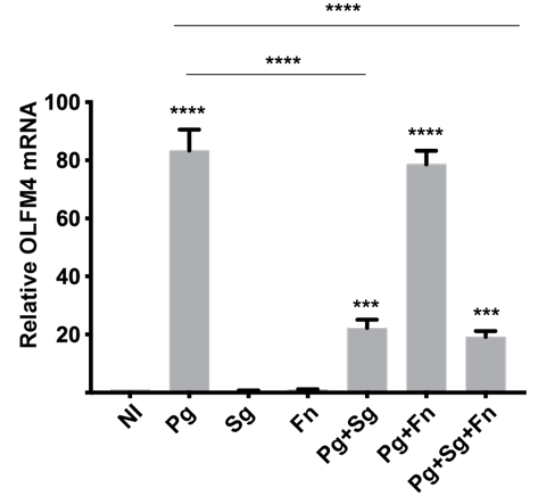

C

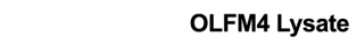

E
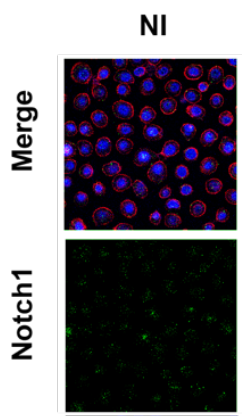

$\frac{\bar{\alpha}}{\Delta}$

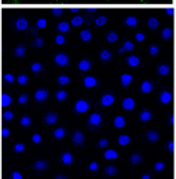

¿্
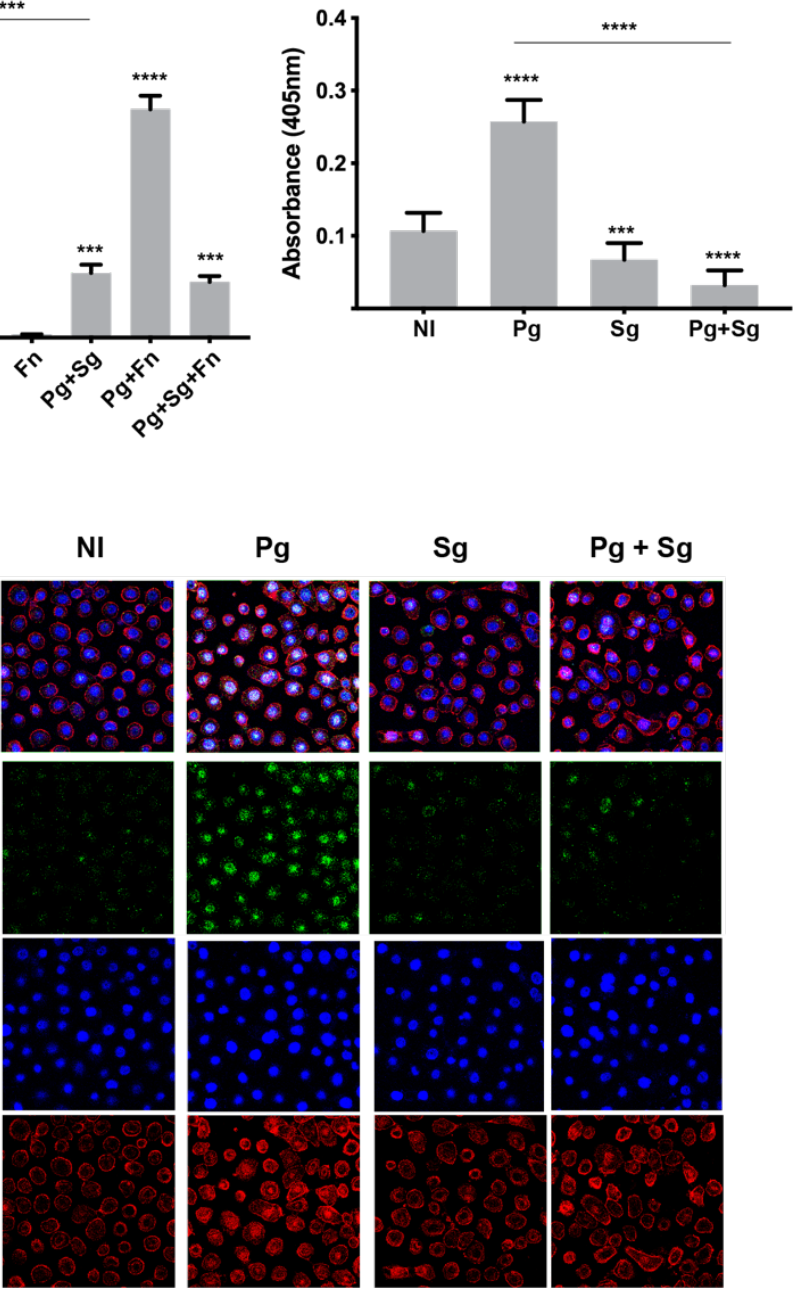

G

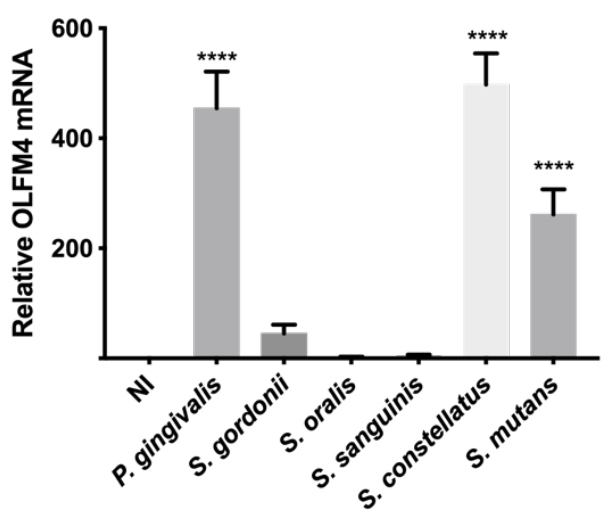

$+P$. gingivalis

Figure 12: Antagonism of P. gingivalis induced OLFM4 by S. gordonii 
$P$. gingivalis is generally found in communities, so we investigated the role these interactions in regulation of OLFM4 in TIGK cells. Cells were challenged with an $\mathrm{MOI}$ of 100 of either $P$. gingivalis and/or Filifactor alocis (Fa), Fusobacterium nucleatum (Fn), Treponema denticola (Td), and Streptococcus gordonii (Sg). Regulation of OLFM4 was unaffected by $\mathrm{Fa}, \mathrm{Fn}$, or Td, but $S$. gordonii significantly reduced OLFM4 regulation by $P$. gingivalis (A). We also investigated the role of multispecies interactions by challenging TIGK cells with Pg (MOI 50), Fn (MOI 50), and Sg (MOI 10), and found that Sg maintained the ability to inhibit OLFM4 (B). Confirmation of protein expression was verified by using either cell lysates (C) or supernatants (D) with an OLFM4 ELISA. P. gingivalis significantly increased protein expression in both cell lysates and supernatants, and $S$. gordonii significantly reduced OLFM4 protein in both cell lysates and supernatants in combination with $P$. gingivalis. Activated Notch localizes to the nucleus, so we then investigated localization of Notch1 with $P$. gingivalis and/or S. gordonii (E). P. gingivalis significantly increased localization of Notch1 to the nucleus, but $S$. gordonii prevented activation and localization of Notch (F). We then investigated the role of other potential oral streptococci in manipulation of $P$. gingivalis signaling. We found that organisms that produce $\mathrm{H}_{2} \mathrm{O}_{2}$ i.e. $S$. gordonii, S. oralis, and S. sanguinis significantly reduced $P$. gingivalis mediated OLFM4 upregulation, whereas Streptococci that are unable to produce $\mathrm{H}_{2} \mathrm{O}_{2}$ showed a reduced ability to inhibit OLFM4 (G). Target genes for qRT-PCR were normalized to GAPDH, and fold changes were calculated using the $\triangle \triangle C T$ method. Significance was calculated using a One-way ANOVA, $* * * \mathrm{P}<.005$, and $* * * * \mathrm{P}<.001$.

However, co-infection with S. gordonii impeded OLFM4 induction by P. gingivalis, and in a three species consortium of $P$. gingivalis, $F$. nucleatum and $S$. gordonii, the antagonistic effect of S. gordonii was dominant (Fig. 12B). We verified OLFM4 expression changes at the protein level using an ELISA, which showed that S. gordonii can antagonize production of OLFM4 as reflected both the intracellular and extracellular amounts of the protein (Fig. 12C-D).

Following cleavage by (gamma)-secretase the intracellular domain of the Notch receptor localizes to the nucleus, where it binds and activates the transcription factor RBP-JK, leading to upregulation of Notch target genes. We utilized confocal microscopy to determine the localization pattern of cleaved Notch in TIGK cells challenged with $P$. gingivalis and/or S. gordonii. Fig. 12E-F shows that $P$. gingivalis induces nuclear 
localization of Notch, which is antagonized in the presence of $S$. gordonii. These results suggest that $S$. gordonii prevents $P$. gingivalis-induced upregulation of OLFM4 by blocking activation of Notch signaling. S. gordonii is one of several species of oral streptococci with the potential to coaggregate with $P$. gingivalis in vivo. A number of additional oral streptococcal species were thus tested for antagonistic properties, and we found that $S$. oralis and S. sanguinis were also capable of inhibiting OLFM4 expression (Fig. 12G). In contrast, S. constellatus and S. mutans did not suppress $P$. gingivalis-induction OLFM4 upregulation. One phenotypic property of these streptococcal species which tracks with antagonism of $P$. gingivalis, is production of hydrogen peroxide $\left(\mathrm{H}_{2} \mathrm{O}_{2}\right)$ as a metabolic by product $[138,139]$.

We have previously shown that $S$. gordonii can antagonize the action of $P$. gingivalis by activating Nemo-like Kinase (NLK) which in turn suppresses the FOXO1 pathway. We confirmed that the antagonistic action of S. gordonii is independent of NLK by siRNA knockdown. As shown in Fig. 13, a reduction in NLK levels did not impact the ability of S. gordonii to prevent $P$. gingivalis induction of OLFM4. Additionally, we investigated the role of exogenous Jag1 in the role of OLFM4 transcriptionally activation, and determined whether S. gordonii could prevent activation through host cell manipulation. Exogenous treatment with recombinant Jag1 was enough to stimulate OLFM4 transcription (Fig. 14), however S. gordonii was unable to impede this activation, which indicates this inhibition could be driven through P. gingivalis-S.gordonii signaling, rather than S. gordonii-host signaling. 


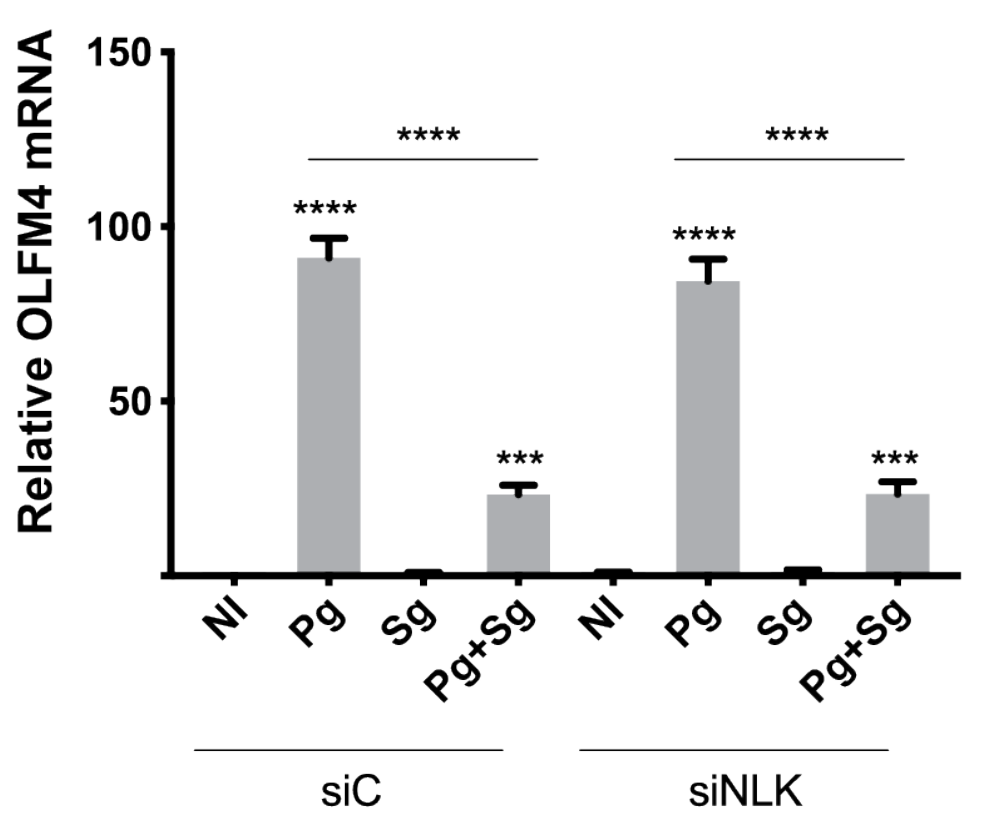

Figure 13: NLK-FOXO1 signaling is not required for inhibition of OLFM4

$S$. gordonii inhibits manipulation of host signaling by $P$. gingivalis through activation of host kinase NLK, which then phosphorylates and inactivates transcription factor Foxo1 (activated by $P$. gingivalis). TIGK cells were transfected with siRNA targeting NLK, then challenged with $P$. gingivalis ( $\mathrm{MOI} 100$ ) and/or S. gordonii (MOI 50) for 24h. RNA was then harvested, and expression of OLFM4 measured by qRT-PCR. Knocking down NLK had no effect on OLFM4 inhibition by S. gordonii. OLFM4 was normalized to GAPDH, and fold changes were calculated using the $\triangle \triangle C T$ method. Significance was calculated using a Oneway ANOVA, $* * * \mathrm{P}<.005$, and $* * * * \mathrm{P}<.001$. 


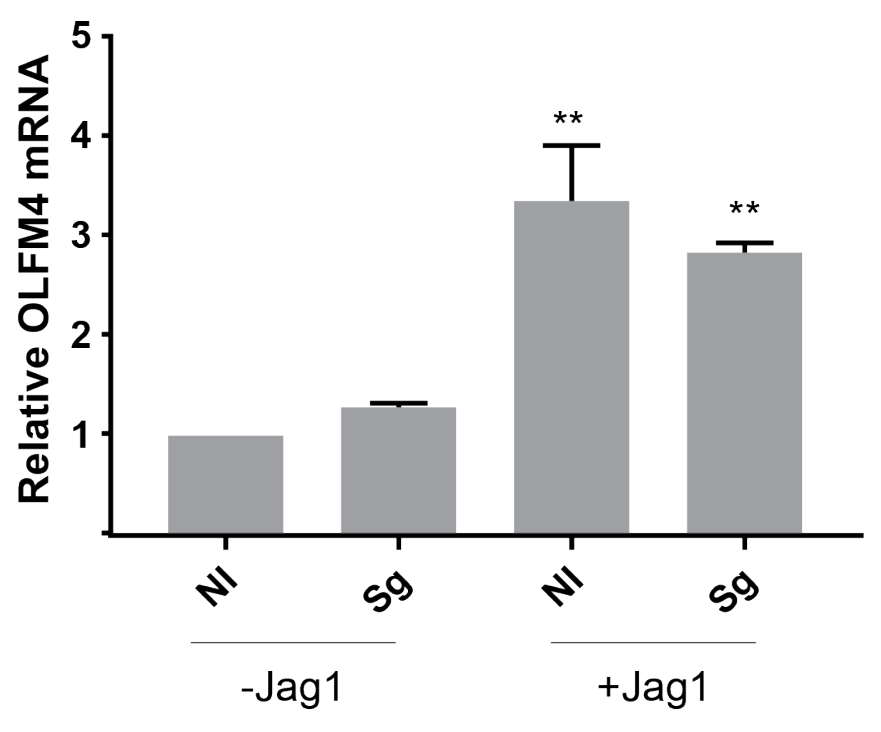

Figure 14: S. gordonii cannot inhibit Notch signaling activated with exogenous Jagged1 TIGK cells were seeded on wells either coated with protein $\mathrm{G}$ or Jag1-FC, then at $24 \mathrm{~h}$ challenged with $S$. gordonii. Exogenous Jag1 was enough to stimulate OLFM4 transcription, however $S$. gordonii was unable to impede this activation. OLFM4 CT values were normalized to GAPDH, and fold changes were calculated using the $\triangle \triangle C T$ method. Significance was calculated using a One-way ANOVA, ${ }^{* *} \mathrm{P}<.01$

To test whether secreted metabolites were sufficient to inhibit OLFM4 expression, we utilized a transwell assay. TIGK cells in the lower chamber were challenged with $P$. gingivalis in the lower chamber along with either S. gordonii, S. oralis, or S. mutans in the upper chamber. Both S. gordonii and S. oralis were able to inhibit $P$. gingivalis-induced OLFM4 expression whereas S. mutans had no effect (Fig. 15A). These results show that functional activity resides in a product secreted from S. gordonii and S. oralis. 
A

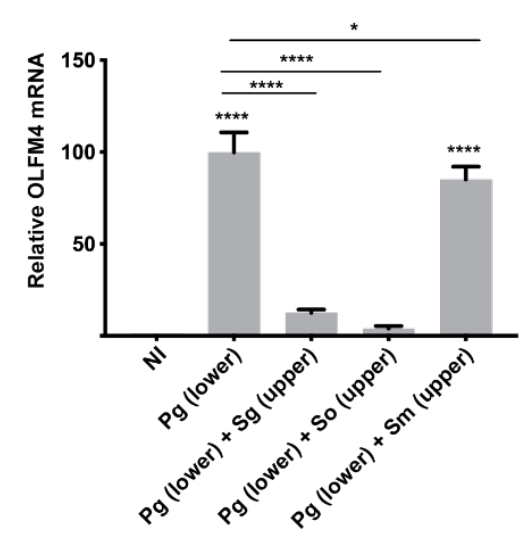

C

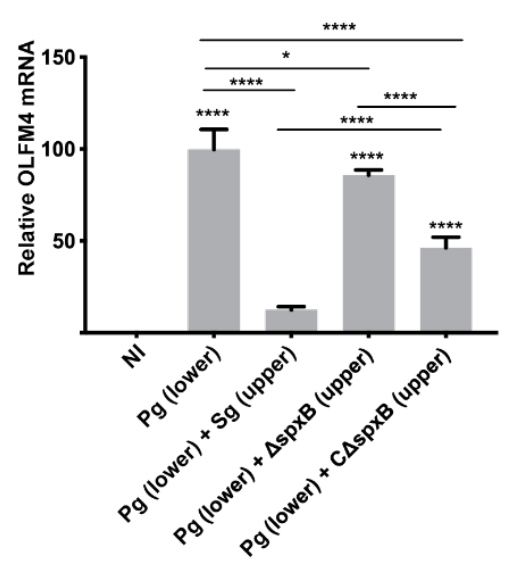

B

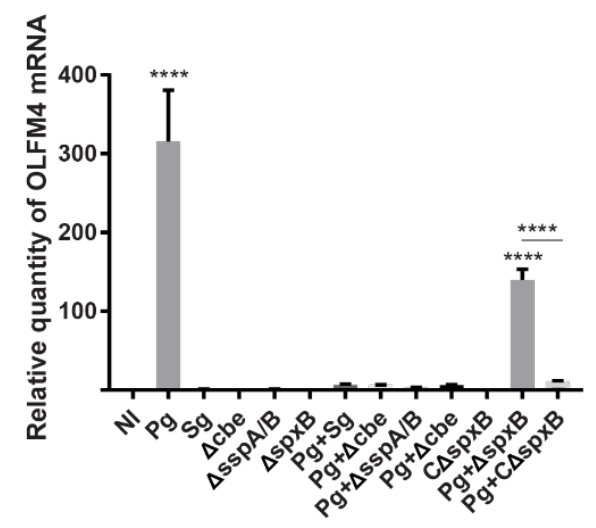

D

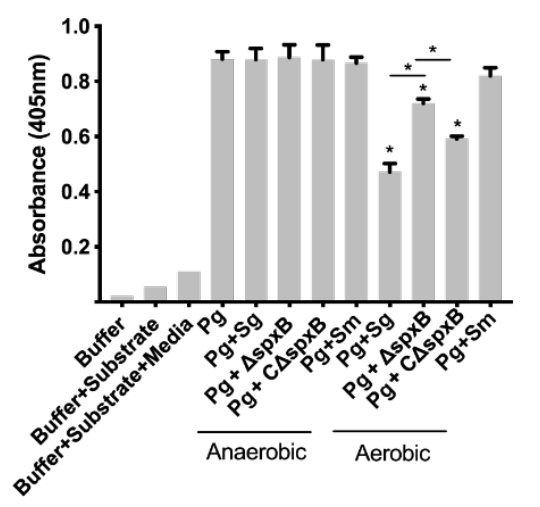

E

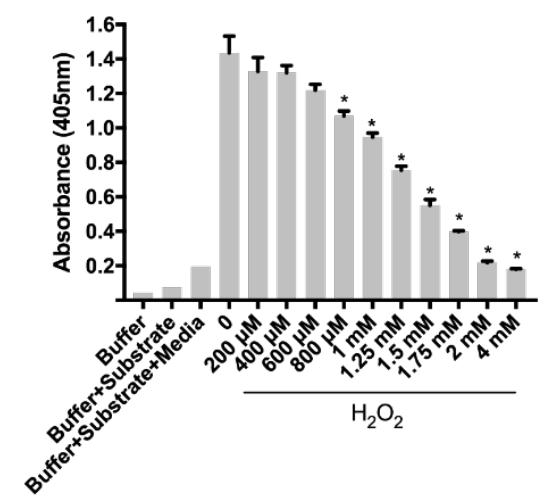

Figure 15: Inhibition of gingipains by Streptococci produced $\mathrm{H}_{2} \mathrm{O}_{2}$

To follow up the role of $\mathrm{H}_{2} \mathrm{O}_{2}$ in the inhibition of OLFM4 by $P$. gingivalis we utilized the 0.4 um transwell system. TIGK cells were grown in the lower chamber, then challenged with $P$. gingivalis ( $\mathrm{MOI} 100$ ) in the lower chamber where indicated and S. gordonii (Sg), S. oralis (So), or S. mutans (Sm) in the upper chamber (A). S. gordonii and S. oralis were able to inhibit OLFM4 independent of contact with either $P$. gingivalis or TIGK cells, and S. mutans did not affect $P$. gingivalis induced OLFM4. S. gordonii interacts with $P$. gingivalis through direct binding with adhesins ( $\mathrm{SspA} / \mathrm{B})$, as well as through secreted metabolites PABA and $\mathrm{H}_{2} \mathrm{O}_{2}$. Inhibition of OLFM4 expression by $P$. gingivalis was not ameliorated with adhesin or pABA secretion deficient mutants $(s s p A / B, c b e)$, however the $\mathrm{H}_{2} \mathrm{O}_{2}$ deficient mutant $(s p x B)$ showed significantly less inhibition. This inhibition was restored by complementing $\operatorname{spxB}(\mathrm{B})$. The role of secreted $\mathrm{H}_{2} \mathrm{O}_{2}$ was confirmed by utilizing 0.4 um transwell filters. TIGK cells were grown in the lower chamber, then challenged with $P$. gingivalis in the lower chamber or the indicated strains of $S$. gordonii in the upper chamber. The deletional $s p x B$ mutant was unable to inhibit OLFM4, whereas the complemented strain was partially restored to wildtype level inhibition (C). Gingipains require a reduced environment for optimal protease activity, so we investigated the role of $\mathrm{H}_{2} \mathrm{O}_{2}$ in oxidizing and therefore inhibiting activity of RgpA/B using a chromogenic substrate assay. Supernatant was taken from $P$. gingivalis grown to mid-log phase by spinning the cells down and filtering the 
supernatant. $P$. gingivalis supernatant was then incubated with metabolically active anaerobic or aerobic $S$. gordonii or $S$. mutans. Supernatant was then collected and filtered, then activity of RgpA/B was measured through cleavage of a chromogenic substrate (D). Anaerobic cultures did not impact activity of RgpA/B, but aerobically grown S. gordonii did reduce activity of RgpA/B by about $50 \%$. This inhibition was ameliorated in the $s p x B$ deletional mutant, and partially restored by the complemented spxB strain. $S$. mutans did not inhibit RgpA/B activity in either anaerobic or aerobic cultures. We then confirmed inhibition of RgpA/B activity by $\mathrm{H}_{2} \mathrm{O}_{2}$ by incubating $P$. gingivalis supernatant with indicated concentrations, and measuring activity by cleavage of a chromogenic substrate. Significant inhibition is observed at $800 \mu \mathrm{m}$, and activity is reduced in a dose dependent manner until $4 \mathrm{mM}$ (E). Target genes for qRT-PCR were normalized to GAPDH, and fold changes were calculated using the $\triangle \triangle C T$ method. Significance was calculated using a One-way ANOVA, and $* \mathrm{P}<.05, * * * \mathrm{P}<.005$, and $* * * * \mathrm{P}<.001$.

To provide additional evidence of a role for $\mathrm{H}_{2} \mathrm{O}_{2}$ we utilized a pyruvate oxidase mutant $(\triangle s p x B)$, in which $\mathrm{H}_{2} \mathrm{O}_{2}$ production is reduced by approximately $90 \%$ through loss of conversion of pyruvate to acetate with $\mathrm{H}_{2} \mathrm{O}_{2}$ as a byproduct. As shown in Fig. $15 \mathrm{~B}$, the $\triangle s p \times B$ mutant was unable to suppress OLFM4 transcription in a co-infection assay. In contrast, mutants of $S$. gordonii unable to bind $(\triangle s s p A / B)$ or accumulate $(\Delta c b e)$ with $P$. gingivalis retained the ability to impede the increase in OLFM4. Similarly, in a transwell assay with $P$. gingivalis in the lower chamber and $S$. gordonii in the upper chamber, the $\triangle s p x B$ mutant had no impact on induction of OLFM4 mRNA by $P$. gingivalis, whereas parental and complemented $\triangle s p x B$ mutant were antagonistic (Fig. 15C). Complementation of the mutation with the $s p x B$ gene in trans $(C \Delta s p x B)$ restored the antagonistic phenotype. Collectively, these results strongly implicate secreted $\mathrm{H}_{2} \mathrm{O}_{2}$ as the effector of streptococcal antagonism of $P$. gingivalis-induced OLFM4 regulation.

Interestingly, gingipains require a reduced environment for activity to maintain the cysteine catalytic domain [140]. Thus, we hypothesized that oxidation by $\mathrm{H}_{2} \mathrm{O}_{2}$ was impairing the activity of the gingipains. To test this, we used a chromogenic assay to 
measure gingipain activity in the supernatant of $P$. gingivalis cultures incubated with streptococci either capable or unable to produce $\mathrm{H}_{2} \mathrm{O}_{2}$ (Fig. 15D). Aerobically cultured $S$. gordonii WT and the complemented mutant $C \triangle s p x B$ significantly reduced gingipain activity. Activity was partially reduced by the $\triangle s p x B$ mutant, possibly due to residual $\mathrm{H}_{2} \mathrm{O}_{2}$ production, and was unaffected by S. mutans. In contrast, following anaerobic culture of the streptococcal strains, in which $\mathrm{H}_{2} \mathrm{O}_{2}$ is not produced, there was no inhibition of gingipain activity in any condition. We then sought to test concentrations of $\mathrm{H}_{2} \mathrm{O}_{2}$ that could inhibit gingipain activity. As shown in Fig. 15E, $800 \mu \mathrm{M}$ was the lowest dose at which significant inhibition of gingipain activity was observed, with the greater reduction occurring between 1-2 mM, which is the range of $\mathrm{H}_{2} \mathrm{O}_{2}$ amounts that can be produced extracellularly by streptococci [141]. Taken together, our data support the concept that $\mathrm{H}_{2} \mathrm{O}_{2}$ produced by certain species of oral streptococci such as $S$. gordonii inhibit the activity of gingipains and consequently impede activation of the Notch pathway by $P$. gingivalis.

Many of the epithelial cell responses to $P$. gingivalis revolve around the axis of survival/proliferation/migration [12]. OLFM4 is an antiapoptotic factor that promotes tumor growth [43]. Thus, we next investigated the extent to which OLFM4 participates in epithelial cell responses to $P$. gingivalis by knocking down OLFM4 and performing RNA Seq. The PCA plot for this RNA seq showed clustering of individual biological replicates in each group, as well as distinct separation between conditions (Fig. 16A). 
A

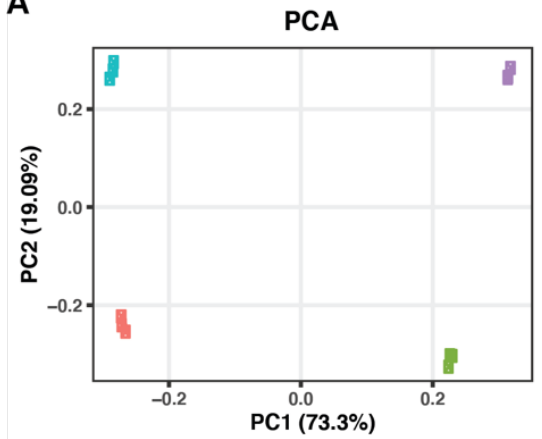

C

siOLFM4+Pg vs. $\mathbf{S i C}+\mathrm{Pg}$ Log2FC cutoff $=1$, adj. $p$-value cutoff $=0.05$
B

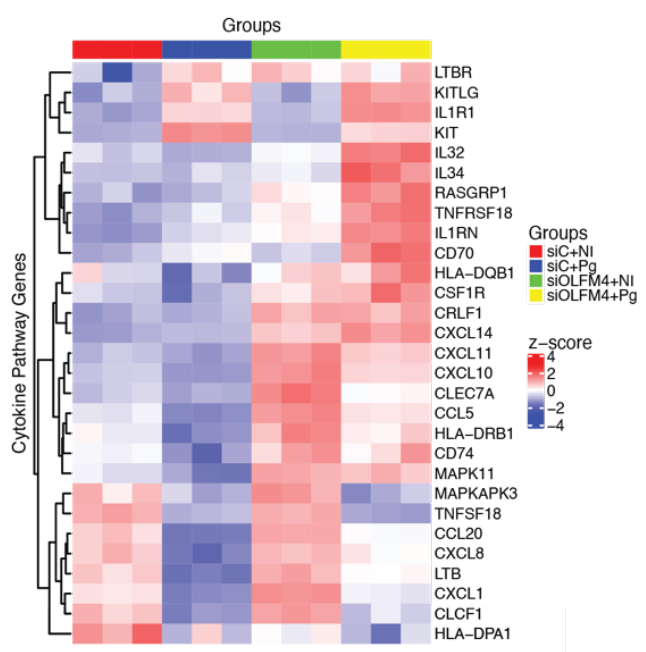

- NS - $\log _{2} F C \bullet p$-value • $\mathrm{p}$-value and $\log _{2} \mathrm{FC}$

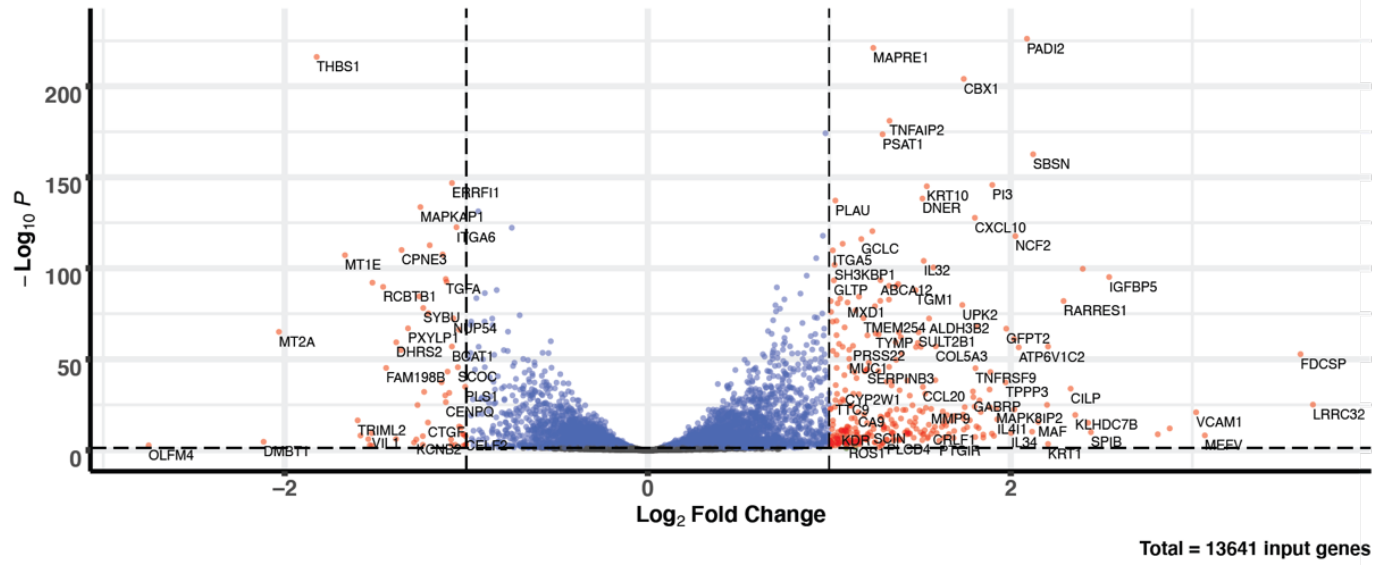

D
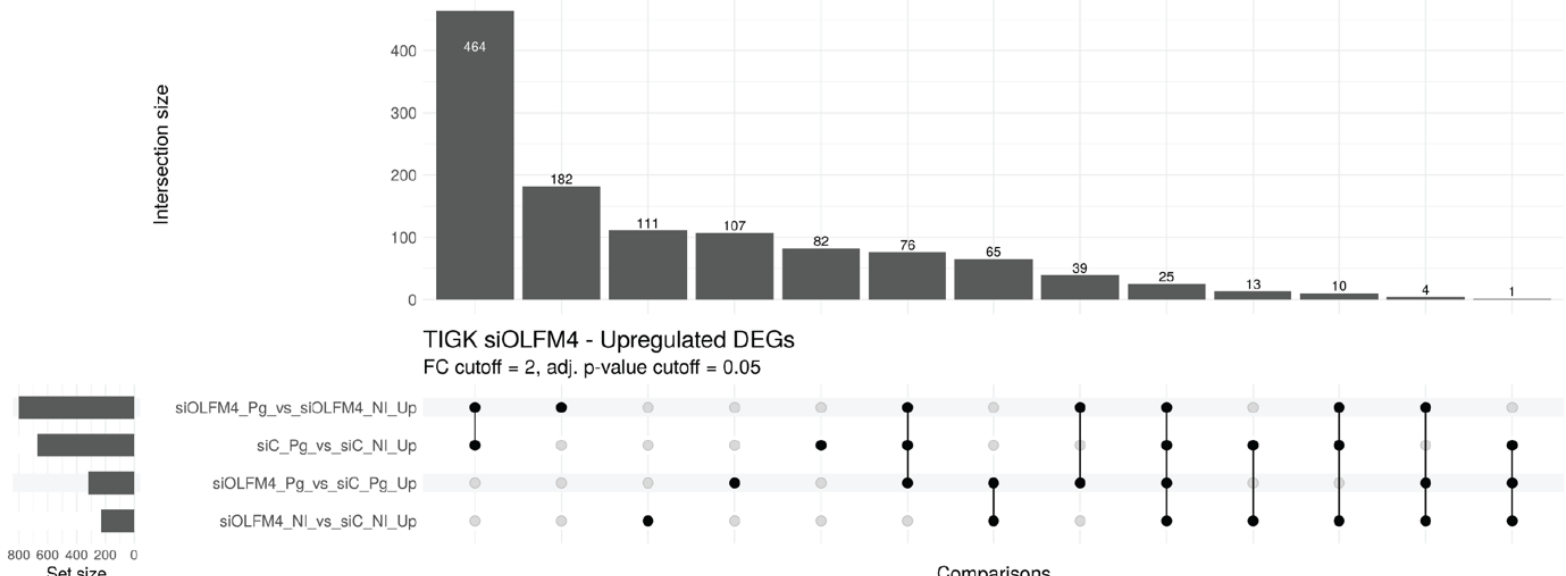

SiOLFM4_Pg_vs_siOLFM4_NI_Up

siC_Pg_vs_siC_NI_Up 


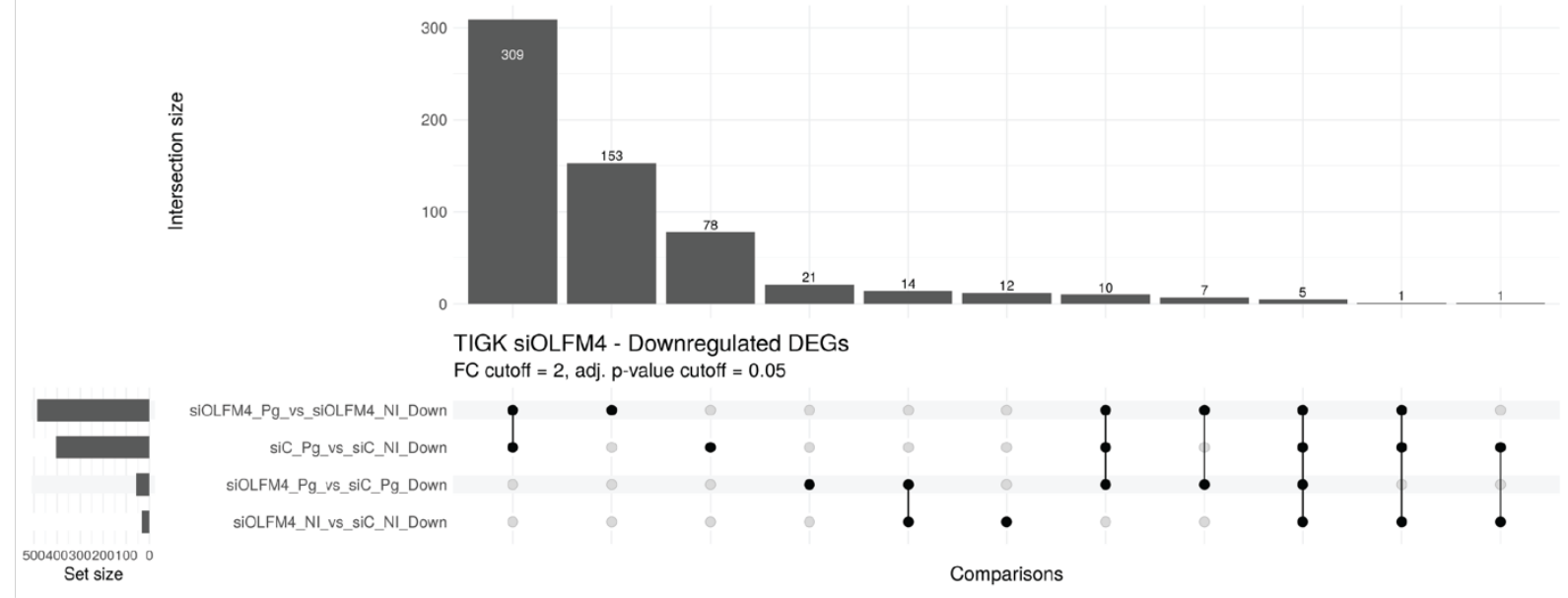

Figure 16: Knocking down OLFM4 reveals altered inflammatory transcriptomic profile TIGK cells were transfected with either scrambled siRNA or siOLFM4, then challenged with P. gingivalis for $24 \mathrm{~h}$. Libraries were prepped as described in Figure 5 . The PCA plot (A) shows tight clustering of individual samples within groups, and distinct clustering of each group. A heatmap of pro-inflammatory cytokines/chemokines reveals significantly upregulated pro-inflammatory markers in the siOLFM4 Pg group compared to the siControl Pg group (B). (C) shows a volcano plot that graphically depicts differential gene expression in siOLFM4 Pg and siControl Pg cells, and (D) summarizes the comparison of each individual group for upregulated and down regulated genes.

Enrichment for pro-inflammatory gene within each group in Fig. 16B revealed a distinct signature in the siOLFM4 conditions, specifically genes that were are known to be downregulated by $P$. gingivalis were upregulated, including CXCL8, CXCL10, and CXCL11. We have summarized the transcriptional changes in the siOLFM4 $P$. gingivalis treated cells and siControl P. gingivalis treated cells as a volcano plot in Fig. $16 \mathrm{C}$ and shared differentially expressed genes are summarized as upset charts in Fig. 16D.

OLFM4 manipulates multiple host processes through protein-protein interactions that $P$. gingivalis is also known to impact, such as migration. We investigated the role for OLFM4 in migration of the epithelial cells. In Fig. 17, we knocked down OLFM4 in epithelial 
cells, then challenged with $P$. gingivalis. We found that reduction of OLFM4 significantly diminished migration of TIGKs in response to $P$. gingivalis. .

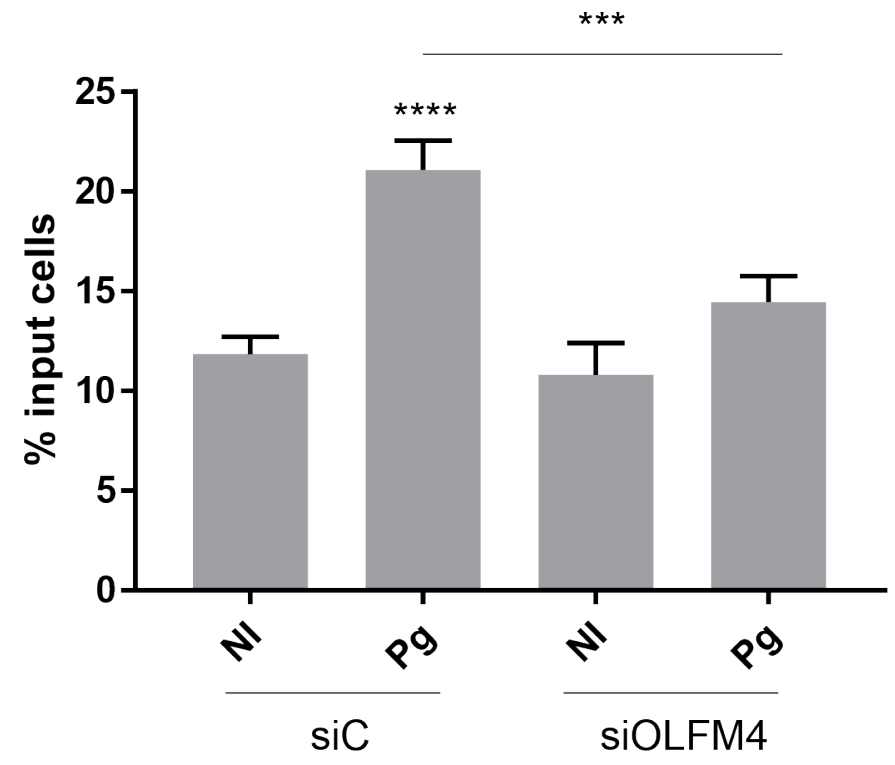

Figure 17: $P$. gingivalis promotes migration of epithelial cells through OLFM4 TIGK cells were transfected with either scrambled siRNA or siRNA targeting OLFM4. $48 \mathrm{~h}$ after transfection cells were challenged with an $\mathrm{MOI}$ of $100 \mathrm{P}$. gingivalis, then seeded over a Matrigel transwell insert for $18 \mathrm{~h}$. After incubation cells were scraped off the surface of the membrane, and the cells that had invaded were fixed with methanol and stained with toluidine blue. Cells were enumerated in at least 3 fields, and percentages calculated in comparison to an insert without Matrigel coating. Significance was calculated using a One-way ANOVA, *** $\mathrm{P}<.005$, and $* * * * \mathrm{P}<.001$.

The regulatory mechanism for OLFM4 by $P$. gingivalis, as well as the method for antagonism by S. gordonii is summarized in Figure 18. 


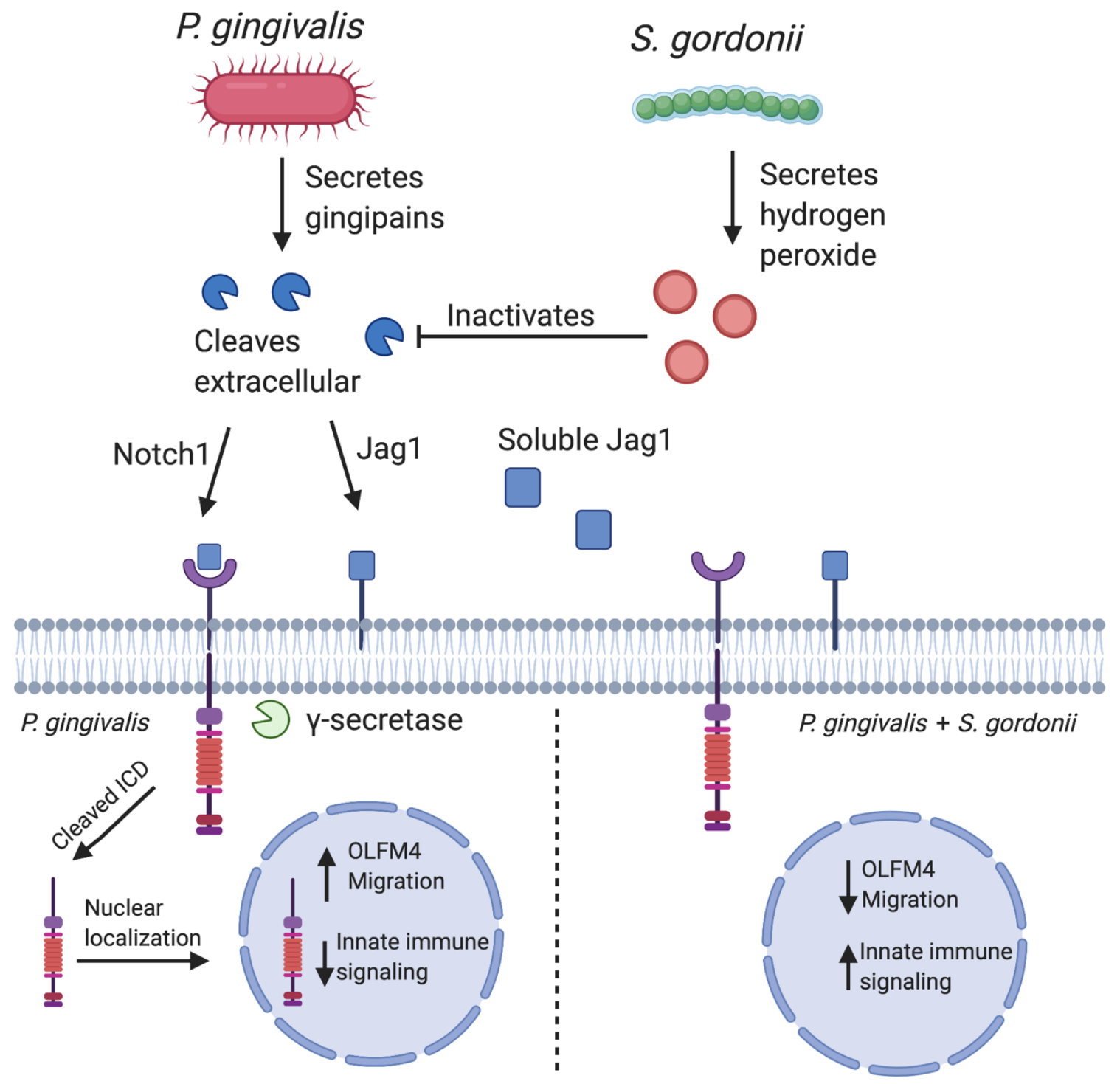

Figure 18: Graphic summarizing the pathway by which $P$. gingivalis activates Notch signaling, and OLFM4 as a downstream target of activation. S. gordonii inhibits this activation through secretion of $\mathrm{H}_{2} \mathrm{O}_{2}$, which inhibits RgpA/B activity, preventing activation and downstream activation of OLFM4. 


\section{CHAPTER 4 DISCUSSION}

The idea of bacteria causing cancer has been fervently studied since the classification of Helicobacter pylori as a causative agent for stomach cancer in 1984. Oral squamous cell carcinoma has a 5 -year mortality rate of approximately $50 \%$, in part because diagnosis tends to be elusive due to its asymptomatic progression. Identification of microorganisms, or a microbial profile, that increases the probability of OSCC would help in identifying those who are high risk, and would represent a unique preventative treatment modality. One candidate bacterium that has been associated with OSCC for decades is $P$. gingivalis, which has the capacity to inhibit apoptosis, enhance proliferation, and induce an epithelial-to-mesenchymal like invasive phenotype in epithelial cells. $P$. gingivalis has been characterized as a keystone pathogen for periodontal disease by its ability to directly manipulate the immune response through its own virulence factors, as well as by driving changes in the microbial community that leads to a dysbiotic relationship with the host immune system, leading to persistent inflammation. This changing microenvironment can be a driving force in the transformation of epithelial cells, as byproducts of inflammation produces a nutrient rich environment and dysregulates the tumor immune microenvironment, which favors persistent colonization by anaerobes. Recent studies have focused on relative abundance of bacteria on sites of OSCC compared to contralateral healthy controls and have reported that numbers of periodontal pathogens like $P$. gingivalis tend to be higher, whereas commensals such as 
some streptococci tend to be decreased [142]. While this likely reflects fitness within the tumor microenvironment, in the absence of homeostatic commensals such as S. gordonii, the tumorigenic potential of $P$. gingivalis will be unconstrained. For example, $P$. gingivalis regulates EMT factor ZEB2 through dephosphorylation of host transcription factor FoxO1. This interaction is antagonized by S. gordonii which induces phosphorylation of FOXO1 through activation of host TAK/NLK kinase cascade. These properties are made more interesting because these organisms interact directly to enhance each other's virulence through attachment and metabolic synergy. However, in the host epithelial environment, these organisms antagonize one another through dysregulation of host signaling cascades.

The goal of this study was to identify signaling events impacted by periodontal pathogen $P$. gingivalis that contribute to homeostatic disruption, specifically focusing on pathways overridden by homeostatic commensal S. gordonii in gingival epithelial cells. We hypothesized that $P$. gingivalis would activate genes involved in tissue disruption and dampening of innate immune signaling, whereas $S$. gordonii would either program the cells to resist this activation or interact with other inhibitory pathways. We approached this by characterizing differences in transcriptomic responses of epithelial cells challenged with $P$. gingivalis and/or S. gordonii using RNAseq, revealing OLFM4, a gene involved in apoptosis, proliferation, migration, and dysregulation of innate immunity, all of which are differentially influenced by the microbes, as a potential axis that is disturbed by $P$. gingivalis and restored by S. gordonii. The initial identification of OLFM4 was followed by 
characterization of the mechanism for upregulation by $P$. gingivalis, inhibition by $S$. gordonii, and ultimately the phenotypic outcome of this differential regulation.

Currently, there are few studies that characterize the complete transcriptional fingerprint left by oral microbes on gingival epithelial cells. Hasegawa et. al. focused on transcriptional changes using a microarray of epithelial cells challenged with either $F$. nucleatum or S. gordonii, which identified activation of MAPK signaling by both organisms [143]. However, they reported that F. nucleatum increased IL-6 and IL-8 production, whereas S. gordonii inhibited production of both. Handfield et. al. focused on the individual challenge of epithelial cells with either $P$. gingivalis or $A$. actinomycetemcomitans [23]. This report showed that $P$. gingivalis activated p53 signaling, leading to inhibition of apoptosis, whereas A. actinomycetemcomitans inactivated p53 signaling, thereby inducing apoptosis [23]. Using gene array analysis in human immortalized gingival keratinocytes (HIGKs), Mans et. al. [144] reported that S. gordonii overrides transcriptional changes in cell cycle regulatory genes, opposing $P$. gingivalis mediated cell cycle progression in favor of cell cycle arrest. While this study provided significant insight into the regulatory mechanisms for cell cycle activity, microarray technology has since been superseded by deep sequencing approaches, and thus new, previously unknown targets can be identified. Additionally, as S. gordonii can antagonize activation of transcription factor FOXO1 by $P$. gingivalis in gingival epithelial cells (discussed above), there are likely to be comprehensive transcriptional changes when $P$. gingivalis and S. gordonii are co-infected. 
In this study, we identified unique complete transcriptomic profiles between gingival epithelial cells challenged with $P$. gingivalis and/or S. gordonii through RNAseq, which characterized the interaction between the co-infected group to be more similar to the individual $S$. gordonii group than the $P$. gingivalis alone group. We started by investigating genes that were differentially regulated by $P$. gingivalis, but the opposite trend was observed in the $P$. gingivalis $+S$. gordonii group. This led us to OLFM4, which was upregulated by $P$. gingivalis, downregulated by S. gordonii, and was significantly reduced compared to the $P$. gingivalis alone group.

OLFM4, also known as GW112, was originally characterized by its function in binding the mitochondrial protein GRIM19 [43], and attenuating interferon- $\beta$ mediated apoptosis in prostate cancer cells [43]. OLFM4 has since been implicated in EMT, cell cycle activity, and modulation of innate immune signaling $[44,45,47,126]$. In non-small cell lung cancer, under hypoxic conditions, OLFM4 is significantly upregulated, and can then positively regulate HIF-1 $\alpha$ to promote EMT [45]. In hepatocellular carcinoma, OLFM4 enhances activation of STAT3, which then increases proliferation and induces EMT in HepG2 cells [145]. H. pylori induces OLFM4 regulation in gastric epithelial cells, which upon stimulation binds NOD1/NOD2, inhibiting activation of NF-KB. Knockout OLFM4 mice could effectively eliminate $H$. pylori from the stomach, whereas wildtype mice experienced persistent colonization [47]. Further investigation revealed a unique cytokine profile in OLFM4 knockout mouse stomach, specifically increased transcriptional and protein levels of IL-1 $\beta$, IL-5, IL-12, and MIP-1 $\alpha$ [47]. In summary, OLFM4 inhibits apoptosis, increases proliferation and invasion, and manipulates host immune signaling, which are 
all characteristic phenotypes of epithelial cells challenged with $P$. gingivalis. Additionally, S. gordonii inhibits $P$. gingivalis induced proliferation and invasion in epithelial cells, which points the potential role for an OLFM4 regulatory axis.

OLFM4 is primarily regulated through the Notch1-Jagged1 signaling cascade. Notch signaling was significantly enriched in the $P$. gingivalis alone RNAseq, with, for example, Notch1, Jagged1, and the Notch target gene Hes5 were upregulated. Interestingly, Notch1, Jagged1, and Hes5 were all significantly lower in the $P$. gingivalis + S. gordonii compared to the $P$. gingivalis alone group. Recently, Al-Attar et. al. found that P. gingivalis activates Notch signaling in oral mucosal and gingival epithelial cells, leading to increased transcription, translation, and secretion of the anti-microbial protein $\mathrm{PLA}_{2}-$ IIA, which is one factor responsible for the dysbiotic shift observed after colonization by P. gingivalis. We similarly observed that $P$. gingivalis induces nuclear localization of Notch1 in epithelial cells. In contrast, not only does $S$. gordonii not induce nuclear localization, but it inhibits Notch localization by $P$. gingivalis. Al-Attar et. al. found that $P$. gingivalis induced nuclear localization of Notch1, whereas S. gordonii and F. nucleatum did not. This study also revealed upregulation of HES1 and HEY1 by $P$. gingivalis in mucosal cells, which is recapitulated in our epithelial cell RNAseq. Additionally, the gingipain deficient $P$. gingivalis could not stimulate Notch1 in OKF6 cells. Previously, OLFM4 had been shown to require Notch1, and stimulation with exogenous Jagged1 in order to increase OLFM4 protein. We corroborated this finding, and additionally we excluded Notch ligands Jagged2 and DLL1-4, as well as Notch2-4 from a role in regulating OLFM4. 
P. gingivalis produced proteolytic enzymes, the $\operatorname{Kgp}, \operatorname{RgpA}$, and $\operatorname{RgpB}$ gingipains, are key virulence factors in the manipulation of host immunity and tissue homeostasis. Each of the gingipains is capable of inhibiting classical, lectin, and alternative pathways of the complement cascade through the proteolytic degradation of $\mathrm{C} 3$. The gingipains also target $\mathrm{C} 5 \mathrm{~b}$, which attenuates the formation of the membrane attack complex. $P$. gingivalis induces non-canonical activation of $\beta$-catenin through cleavage by the gingipains into functional fragments, which are able to translocate to the nucleus. Functional $\beta$-catenin and gingipains are required for upregulation of ZEB2, and $\beta$-catenin is also likely involved in the regulation of proliferation and production of MMP7. Additionally, $P$. gingivalis cleaves MMP-1, -3 , and -9 into their active forms with the gingipains, which leads to increased destruction of the host periodontium and potentially increased motility of epithelial cells. $P$. gingivalis induces a local chemokine paralysis through degradation of CXCL8, CXCL9, and CXCL10, which is mediated through a secreted proteolytic enzyme (likely the gingipains.) Additionally, the gingipains have been shown to proteolytically degrade $\beta$-catenin into its functional form, which is capable of nuclear localization. This proteolytic activation was capable of being induced with purified gingipains, in the absence of any whole cell $P$. gingivalis whole cells. $\beta$-catenin regulates cell proliferation, as well as MMPs, which implicates an additional role for the gingipains in these phenotypes. $P$. gingivalis gingipains activate PAR2 and PAR4, leading to the phosphorylation of IKB, the nuclear translocation of NF-KB, and increased transcription of proMMP9 $(50,51)$. In addition, gingipains can cleave proMMP9, generating the mature active enzyme (52), which is important for cancer cell invasion and metastasis. Gingipains 
can also proteolytically process proteins on the epithelial cell surface, causing release and redistribution, with consequent effects on signal transduction and inflammatory responses $(53,54)$. Intracellularly, $P$. gingivalis gingipains can degrade mammalian target of rapamycin (mTOR), thus disrupting the mTOR pathway which regulates the cytoskeleton, as well as cleave $\beta$-actin directly $(55,56)$. In trophoblasts, gingipains can degrade $\mathrm{p} 53$ and the $\mathrm{E} 3$ ubiquitin protein ligase homolog protein (MDM2) and modulate the activity of multiple signaling pathways, resulting in both cell cycle arrest and cell death (57). The capacity of gingipains to regulate the expression of inflammatory mediators at the mRNA level has also been demonstrated. In gingival fibroblasts, gingipains increase TGF $\beta$ gene expression, while suppressing the expression of CXCL8 (58). Elevated signaling through TGF $\beta$ and STAT3 could also contribute to tumorigenesis.

We found here that the gingipains are required for the activation of Notch signaling through a previously unknown mechanism. Our data support a role for each of the gingipains in cleaving the transmembrane protein Jagged1 into a soluble form, allowing for paracrine activation of Notch signaling, as well as acting in place of the extracellular ADAM10/17 proteases in cleaving Notch1 into its active form. We propose a role for OLFM4 in the regulation of invasion in gingival epithelial cells, as well as dampens epithelial pro-inflammatory genes, which are both functions that require gingipains. Fig. 19 integrates OLFM4 signaling into the context of other epithelial cell signaling pathways dysregulated by $P$. gingivalis. 


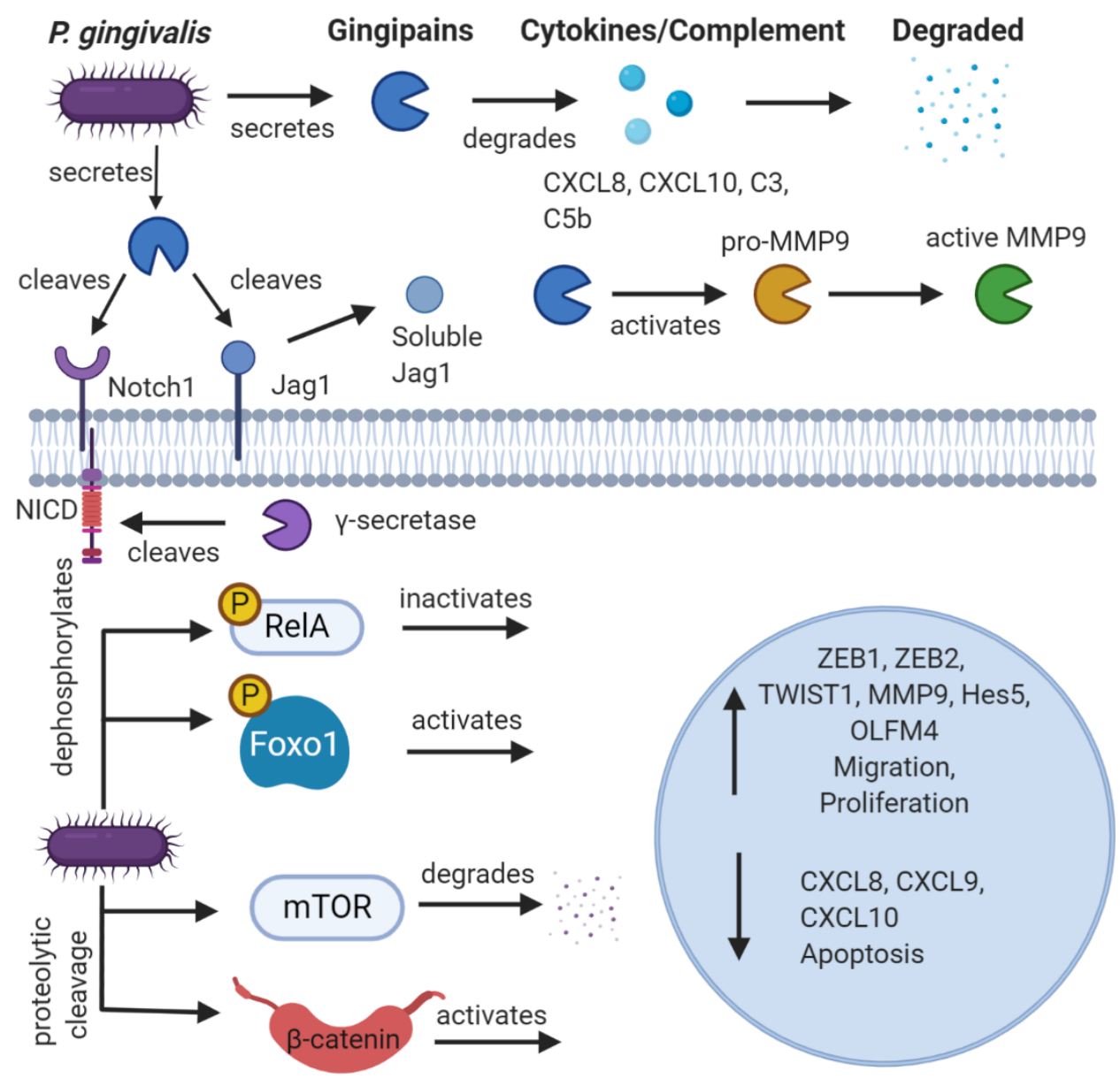

Figure 19: Graphic summarizing signaling pathways manipulated by $P$. gingivalis

While studying organisms individually reveals phenotypes specific to the organisms, studying interactions in the context of communities provides greater biological relevance, as it is more similar to the organization of microbes in the oral cavity. Communities in the oral cavity tend to be complex, reducing the possibility for rogue, individual species to interact with host cells. There are many advantages to this lifestyle, such as protection from environmental stresses, metabolic cross-feeding, antibiotic resistance, increased protection from immune surveillance, and an increased ability to colonize the host through binding the biofilm substratum and community members 
through adhesins. P. gingivalis interacts with multiple bacteria in the oral cavity, specifically F. alocis, S. gordonii, and T. denticola. A key feature for colonization is the ability to attach to members of the community, and benefit each other through metabolic cross-feeding. F. alocis is an emerging pathogen that is capable of localizing to gingival epithelial cells, as well as attaching to and developing a heterotypic community with $P$. gingivalis. These organisms have opposing interactions with epithelial cells, specifically in regards to apoptosis and inflammation. Similar to F. alocis, T. denticola also interacts with epithelial cells and $P$. gingivalis. $T$. denticola has been shown to interact with epithelial cells through the secretion of a chymotrypsin like protease capable of degrading host cytokines and activating MMPs. Interestingly, this protease was not sufficient to activate Notch signaling in gingival epithelial cells, alluding to a more specific interactions being required for Notch1 cleavage by $P$. gingivalis.

As a primary colonizer, S. gordonii interacts with a multitude of organisms. $S$. gordonii plays a key role in the structure and regulation of pathogenicity in oral biofilms through its secretion of $\mathrm{H}_{2} \mathrm{O}_{2}$. While this would normally be toxic to the anaerobic organisms that are prevalent in periodontal diseases, there are organisms within the biofilm that are able to detoxify $\mathrm{H}_{2} \mathrm{O}_{2}$, such as $A$. actinomycetemcomitans. $A$. actinomycetemcomitans senses $\mathrm{H}_{2} \mathrm{O}_{2}$ through the oxygen sensitive OxyR transcriptional regulator, which then activates catalase (KatA) and Dispersin $\mathrm{B}$. Catalase detoxifies $\mathrm{H}_{2} \mathrm{O}_{2}$, and Dispersin B is used to optimally distance itself from S. gordonii. Oral streptococci can be stratified into health-associated species that produce $\mathrm{H}_{2} \mathrm{O}_{2}$, such as $\mathrm{S}$. gordonii and $\mathrm{S}$. oralis, and cariogenic species that do not produce $\mathrm{H}_{2} \mathrm{O}_{2}$, such as S. mutans. S. gordonii and 
S. oralis contribute to a healthy community through $\mathrm{H}_{2} \mathrm{O}_{2}$ induced cell death of $\mathrm{S}$. mutans [146]. Liu et. al. characterized the production of $\mathrm{H}_{2} \mathrm{O}_{2}$ in a biofilm by S. gordonii, specifically finding that $\mathrm{H}_{2} \mathrm{O}_{2}$ is produced at $0.7 \mathrm{mM}-1.6 \mathrm{mM}$ over a period of $2-8 \mathrm{~h}$ which is sufficient to significantly impair RgpA/B activity, with inhibition starting at $0.8 \mathrm{mM}$ and maximal inhibition occurring at $2 \mathrm{mM}$. Not only does $\mathrm{H}_{2} \mathrm{O}_{2}$ modulate the composition of biofilms, but it is also impacts survival and pathogenicity of other organisms. In defined media, $P$. gingivalis survives in up to $3 \mathrm{mM}$ of $\mathrm{H}_{2} \mathrm{O}_{2}$, with $\mathrm{S}$. gordonii and $\mathrm{S}$. oralis producing $\mathrm{H}_{2} \mathrm{O}_{2}$ at about 1-2 mM. P. gingivalis has two characterized tyrosine phosphatases, which respond differently to $\mathrm{H}_{2} \mathrm{O}_{2}$. Ltp1 activates the transcriptional regulator $\mathrm{CdhR}$, which inhibits mfa1 and luxS, having an overall net reduction in community development. $\mathrm{H}_{2} \mathrm{O}_{2}$ inhibits Ltp1 activity, inactivating CdhR, promoting mfa1 and luxS, leading to an overall net gain in community development. Additionally, $P$. gingivalis controls community development with S. gordonii through another tyrosine phosphatase Php1. Php1 positively regulates exopolysaccharide (EPS) production, which is an important feature of biofilm substrata, acting a glue for monospecies and multispecies interactions. This enzyme dephosphorylates Ptk1, a $P$. gingivalis tyrosine kinase, which is another key regulator of EPS production and community development.

Gingipains are cysteine proteases that require a reducing environment for optimal activity. Because $\mathrm{H}_{2} \mathrm{O}_{2}$ would oxidize the disulfide bonds, leading to inactivation of the gingipains, we characterized and titrated this inhibition. Consistent with our hypothesis, the gingipains were inactivated by the $\mathrm{H} 2 \mathrm{O} 2$-producing S. gordonii, but not S. mutans. This inhibition of gingipain activity is pivotal for the antagonism of Notch activation by $S$. 
gordonii. The communication via secreted metabolites is interesting in that there needn't be direct contact with the microbes to each other, or to the epithelial cells.

With an increased interest in pathogenic microorganisms, or microbial profiles, that can act as carcinogenic agents, investigators up until now have generally overlooked the role of commensal organisms maintaining homeostatic interactions in the host. Here we provide additional evidence for the role of S. gordonii as a homeostatic commensal in the context of epithelial cell transformation, whereas in periodontal disease it is considered an accessory pathogen. This system is unique in that these organisms do not necessarily need to be directly associated with the epithelial cells, but instead can mediate host signaling through secreted proteases/metabolites. It's particularly interesting that $P$. gingivalis can perpetuate Notch signaling through cleavage of Jagged1, so cells that had never interacted with $P$. gingivalis are able to induce Notch signaling and OLFM4 expression. Interestingly, recent studies have found that not only is $P$. gingivalis significantly enriched in OSCC, but Streptococci are significantly underrepresented under the same conditions. This study provides insight on the complex molecular dialogue between the commensals and pathogens of the microbiome, and particularly expands on the role of $S$. gordonii through direct inactivation of $P$. gingivalis secreted proteases, in addition to the previously studied subversion of host pathways to impede $P$. gingivalis induced signaling. 


\section{REFERENCES}

1. Marsh, D., et al., Stromal features are predictive of disease mortality in oral cancer patients. J Pathol, 2011. 223(4): p. 470-81.

2. Markopoulos, A.K., Current aspects on oral squamous cell carcinoma. Open Dent J, 2012. 6: p. 126-30.

3. Ganly, I., et al., Periodontal pathogens are a risk factor of oral cavity squamous cell carcinoma, independent of tobacco and alcohol and human papillomavirus. Int J Cancer, 2019. 145(3): p. 775-784.

4. Bagan, J., G. Sarrion, and Y. Jimenez, Oral cancer: clinical features. Oral Oncol, 2010. 46(6): p. 414-7.

5. Seoane, J., et al., Gingival squamous cell carcinoma: diagnostic delay or rapid invasion? J Periodontol, 2006. 77(7): p. 1229-33.

6. Meyer, M.S., et al., A review of the relationship between tooth loss, periodontal disease, and cancer. Cancer Causes Control, 2008. 19(9): p. 895-907.

7. Shi, J., et al., Tooth loss and cancer risk: a dose-response meta analysis of prospective cohort studies. Oncotarget, 2018. 9(19): p. 15090-15100.

8. Katz, J., et al., Presence of Porphyromonas gingivalis in gingival squamous cell carcinoma. Int J Oral Sci, 2011. 3(4): p. 209-15.

9. Chang, C., et al., The prevalence rate of periodontal pathogens and its association with oral squamous cell carcinoma. Appl Microbiol Biotechnol, 2019. 103(3): p. 1393-1404.

10. Nieminen, M.T., et al., Treponema denticola chymotrypsin-like proteinase may contribute to orodigestive carcinogenesis through immunomodulation. $\mathrm{Br} \mathrm{J}$ Cancer, 2018. 118(3): p. 428-434.

11. Polk, D.B. and R.M. Peek, Jr., Helicobacter pylori: gastric cancer and beyond. Nat Rev Cancer, 2010. 10(6): p. 403-14.

12. Whitmore, S.E. and R.J. Lamont, Oral bacteria and cancer. PLoS Pathog, 2014. 10(3): p. e1003933.

13. Garrett, W.S., Cancer and the microbiota. Science, 2015. 348(6230): p. 80-6.

14. Hajishengallis, G., et al., Low-abundance biofilm species orchestrates inflammatory periodontal disease through the commensal microbiota and complement. Cell Host Microbe, 2011. 10(5): p. 497-506.

15. Lamont, R.J. and G. Hajishengallis, Polymicrobial synergy and dysbiosis in inflammatory disease. Trends Mol Med, 2015. 21(3): p. 172-83.

16. Gao, S., et al., Presence of Porphyromonas gingivalis in esophagus and its association with the clinicopathological characteristics and survival in patients with esophageal cancer. Infect Agent Cancer, 2016. 11: p. 3. 
17. Sztukowska, M.N., et al., Porphyromonas gingivalis initiates a mesenchymal-like transition through ZEB1 in gingival epithelial cells. Cell Microbiol, 2016. 18(6): p. 844-58.

18. Lee, J., et al., A novel kinase function of a nucleoside-diphosphate-kinase homologue in Porphyromonas gingivalis is critical in subversion of host cell apoptosis by targeting heat-shock protein 27. Cell Microbiol, 2018. 20(5): p. e12825.

19. Takeuchi, H., et al., Intracellular periodontal pathogen exploits recycling pathway to exit from infected cells. Cell Microbiol, 2016. 18(7): p. 928-48.

20. Tribble, G.D., et al., A Porphyromonas gingivalis haloacid dehalogenase family phosphatase interacts with human phosphoproteins and is important for invasion. Proc Natl Acad Sci U S A, 2006. 103(29): p. 11027-32.

21. Geng, F., et al., Persistent Exposure to Porphyromonas gingivalis Promotes Proliferative and Invasion Capabilities, and Tumorigenic Properties of Human Immortalized Oral Epithelial Cells. Front Cell Infect Microbiol, 2017. 7: p. 57.

22. Geng, F., et al., Identification of Potential Candidate Genes of Oral Cancer in Response to Chronic Infection With Porphyromonas gingivalis Using Bioinformatical Analyses. Front Oncol, 2019. 9: p. 91.

23. Handfield, M., et al., Distinct transcriptional profiles characterize oral epitheliummicrobiota interactions. Cell Microbiol, 2005. 7(6): p. 811-23.

24. Mao, S., et al., Intrinsic apoptotic pathways of gingival epithelial cells modulated by Porphyromonas gingivalis. Cell Microbiol, 2007. 9(8): p. 1997-2007.

25. Yilmaz, O., et al., Activation of the phosphatidylinositol 3-kinase/Akt pathway contributes to survival of primary epithelial cells infected with the periodontal pathogen Porphyromonas gingivalis. Infect Immun, 2004. 72(7): p. 3743-51.

26. Yao, L., et al., Porphyromonas gingivalis infection sequesters pro-apoptotic Bad through Akt in primary gingival epithelial cells. Mol Oral Microbiol, 2010. 25(2): p. 89-101.

27. Moffatt, C.E. and R.J. Lamont, Porphyromonas gingivalis induction of microRNA203 expression controls suppressor of cytokine signaling 3 in gingival epithelial cells. Infect Immun, 2011. 79(7): p. 2632-7.

28. Yilmaz, O., et al., ATP scavenging by the intracellular pathogen Porphyromonas gingivalis inhibits P2X7-mediated host-cell apoptosis. Cell Microbiol, 2008. 10(4): p. 863-75.

29. Lee, K., et al., Porphyromonas gingivalis traffics into endoplasmic reticulum-richautophagosomes for successful survival in human gingival epithelial cells. Virulence, 2018. 9(1): p. 845-859.

30. Wang, Q., et al., FOXO responses to Porphyromonas gingivalis in epithelial cells. Cell Microbiol, 2015. 17(11): p. 1605-17.

31. Kuboniwa, M., et al., P. gingivalis accelerates gingival epithelial cell progression through the cell cycle. Microbes Infect, 2008. 10(2): p. 122-8.

32. Zhou, Y., et al., Noncanonical activation of beta-catenin by Porphyromonas gingivalis. Infect Immun, 2015. 83(8): p. 3195-203. 
33. Chang, C., et al., Porphyromonas gingivalis Infection Promoted the Proliferation of Oral Squamous Cell Carcinoma Cells through the miR-21/PDCD4/AP-1

Negative Signaling Pathway. ACS Infect Dis, 2019. 5(8): p. 1336-1347.

34. Hoppe, T., et al., Oral pathogens change proliferation properties of oral tumor cells by affecting gene expression of human defensins. Tumour Biol, 2016. 37(10): p. 13789-13798.

35. Abdulkareem, A.A., et al., Periodontal pathogens promote epithelialmesenchymal transition in oral squamous carcinoma cells in vitro. Cell Adh Migr, 2018. 12(2): p. 127-137.

36. Ha, N.H., et al., Prolonged and repetitive exposure to Porphyromonas gingivalis increases aggressiveness of oral cancer cells by promoting acquisition of cancer stem cell properties. Tumour Biol, 2015. 36(12): p. 9947-60.

37. Lee, J., et al., Human Primary Epithelial Cells Acquire an Epithelial-MesenchymalTransition Phenotype during Long-Term Infection by the Oral Opportunistic Pathogen, Porphyromonas gingivalis. Front Cell Infect Microbiol, 2017. 7: p. 493.

38. Ohshima, J., et al., Streptococcus gordonii programs epithelial cells to resist ZEB2 induction by Porphyromonas gingivalis. Proc Natl Acad Sci U S A, 2019. 116(17): p. 8544-8553.

39. Ha, N.H., et al., Porphyromonas gingivalis increases the invasiveness of oral cancer cells by upregulating IL-8 and MMPs. Cytokine, 2016. 86: p. 64-72.

40. Inaba, H., et al., Porphyromonas gingivalis promotes invasion of oral squamous cell carcinoma through induction of proMMP9 and its activation. Cell Microbiol, 2014. 16(1): p. 131-45.

41. Inaba, H., et al., Involvement of protease-activated receptor 4 in over-expression of matrix metalloproteinase 9 induced by Porphyromonas gingivalis. Med Microbiol Immunol, 2015. 204(5): p. 605-12.

42. Al-Attar, A., et al., Activation of Notch-1 in oral epithelial cells by P. gingivalis triggers the expression of the antimicrobial protein PLA2-IIA. Mucosal Immunol, 2018. 11(4): p. 1047-1059.

43. Zhang, X., et al., GW112, a novel antiapoptotic protein that promotes tumor growth. Cancer Res, 2004. 64(7): p. 2474-81.

44. Kobayashi, D., et al., Olfactomedin 4 promotes $S$-phase transition in proliferation of pancreatic cancer cells. Cancer Sci, 2007. 98(3): p. 334-40.

45. Gao, X.Z., et al., Blocking OLFM4/HIF-1alpha axis alleviates hypoxia-induced invasion, epithelial-mesenchymal transition, and chemotherapy resistance in non-small-cell lung cancer. J Cell Physiol, 2019.

46. Ye, L., et al., Prognostic Significance and Functional Relevance of Olfactomedin 4 in Early-Stage Hepatocellular Carcinoma. Clin Transl Gastroenterol, 2020. 11(1): p. e00124.

47. Liu, W., et al., Olfactomedin 4 down-regulates innate immunity against Helicobacter pylori infection. Proc Natl Acad Sci U S A, 2010. 107(24): p. 1105661. 
48. Marimuthu, A., et al., Identification of head and neck squamous cell carcinoma biomarker candidates through proteomic analysis of cancer cell secretome. Biochim Biophys Acta, 2013. 1834(11): p. 2308-16.

49. Sahingur, S.E. and W.A. Yeudall, Chemokine function in periodontal disease and oral cavity cancer. Front Immunol, 2015. 6: p. 214.

50. Michaud, D.S., et al., Periodontal Disease, Tooth Loss, and Cancer Risk. Epidemiol Rev, 2017. 39(1): p. 49-58.

51. Groeger, S., et al., B7-H1 and B7-DC receptors of oral squamous carcinoma cells are upregulated by Porphyromonas gingivalis. Immunobiology, 2011. 216(12): p. 1302-10.

52. Yee, M., et al., Porphyromonas gingivalis stimulates IL-6 and IL-8 secretion in GMSM-K, HSC-3 and H413 oral epithelial cells. Anaerobe, 2014. 28: p. 62-7.

53. Joh, T., et al., Helicobacter pylori-stimulated interleukin-8 (IL-8) promotes cell proliferation through transactivation of epidermal growth factor receptor (EGFR) by disintegrin and metalloproteinase (ADAM) activation. Dig Dis Sci, 2005. 50(11): p. 2081-9.

54. Grivennikov, S.I., et al., Adenoma-linked barrier defects and microbial products drive IL-23/IL-17-mediated tumour growth. Nature, 2012. 491(7423): p. 254-8.

55. Cheng, W.C., et al., Periodontitis-associated pathogens $P$. gingivalis and $A$. actinomycetemcomitans activate human CD14(+) monocytes leading to enhanced Th17/IL-17 responses. Eur J Immunol, 2016. 46(9): p. 2211-21.

56. Darveau, R.P., et al., Local chemokine paralysis, a novel pathogenic mechanism for Porphyromonas gingivalis. Infect Immun, 1998. 66(4): p. 1660-5.

57. Takeuchi, H., et al., The serine phosphatase SerB of Porphyromonas gingivalis suppresses IL-8 production by dephosphorylation of NF-kappaB ReIA/p65. PLoS Pathog, 2013. 9(4): p. e1003326.

58. Jauregui, C.E., et al., Suppression of T-cell chemokines by Porphyromonas gingivalis. Infect Immun, 2013. 81(7): p. 2288-95.

59. Wu, J.S., et al., Porphyromonas gingivalis Promotes 4-Nitroquinoline-1-OxideInduced Oral Carcinogenesis With an Alteration of Fatty Acid Metabolism. Front Microbiol, 2018. 9: p. 2081.

60. Binder Gallimidi, A., et al., Periodontal pathogens Porphyromonas gingivalis and Fusobacterium nucleatum promote tumor progression in an oral-specific chemical carcinogenesis model. Oncotarget, 2015. 6(26): p. 22613-23.

61. Wen, L., et al., Porphyromonas gingivalis Promotes Oral Squamous Cell Carcinoma Progression in an Immune Microenvironment. J Dent Res, 2020. 99(6): p. 666-675.

62. Stashenko, P., et al., The Oral Mouse Microbiome Promotes Tumorigenesis in Oral Squamous Cell Carcinoma. mSystems, 2019. 4(4).

63. Han, Y.W., et al., Periodontal disease, atherosclerosis, adverse pregnancy outcomes, and head-and-neck cancer. Adv Dent Res, 2014. 26(1): p. 47-55.

64. Xu, M., et al., FadA from Fusobacterium nucleatum utilizes both secreted and nonsecreted forms for functional oligomerization for attachment and invasion of host cells. J Biol Chem, 2007. 282(34): p. 25000-9. 
65. Rubinstein, M.R., et al., Fusobacterium nucleatum promotes colorectal carcinogenesis by modulating E-cadherin/beta-catenin signaling via its FadA adhesin. Cell Host Microbe, 2013. 14(2): p. 195-206.

66. Rubinstein, M.R., et al., Fusobacterium nucleatum promotes colorectal cancer by inducing Wnt/beta-catenin modulator Annexin A1. EMBO Rep, 2019. 20(4).

67. Abed, J., et al., Fap2 Mediates Fusobacterium nucleatum Colorectal Adenocarcinoma Enrichment by Binding to Tumor-Expressed Gal-GaINAc. Cell Host Microbe, 2016. 20(2): p. 215-25.

68. Gur, C., et al., Binding of the Fap2 protein of Fusobacterium nucleatum to human inhibitory receptor TIGIT protects tumors from immune cell attack. Immunity, 2015. 42(2): p. 344-355.

69. Gur, C., et al., Fusobacterium nucleatum supresses anti-tumor immunity by activating CEACAM1. Oncoimmunology, 2019. 8(6): p. e1581531.

70. Bui, F.Q., et al., Fusobacterium nucleatum infection of gingival epithelial cells leads to NLRP3 inflammasome-dependent secretion of IL-1beta and the danger signals ASC and HMGB1. Cell Microbiol, 2016. 18(7): p. 970-81.

71. Uitto, V.J., et al., Fusobacterium nucleatum increases collagenase 3 production and migration of epithelial cells. Infect Immun, 2005. 73(2): p. 1171-9.

72. Kostic, A.D., et al., Fusobacterium nucleatum potentiates intestinal tumorigenesis and modulates the tumor-immune microenvironment. Cell Host Microbe, 2013. 14(2): p. 207-15.

73. Dashper, S.G., et al., Virulence factors of the oral spirochete Treponema denticola. J Dent Res, 2011. 90(6): p. 691-703.

74. Narikiyo, M., et al., Frequent and preferential infection of Treponema denticola, Streptococcus mitis, and Streptococcus anginosus in esophageal cancers. Cancer Sci, 2004. 95(7): p. 569-74.

75. Fenno, J.C., Treponema denticola interactions with host proteins. J Oral Microbiol, 2012. 4.

76. Listyarifah, D., et al., Treponema denticola chymotrypsin-like proteinase is present in early-stage mobile tongue squamous cell carcinoma and related to the clinicopathological features. J Oral Pathol Med, 2018. 47(8): p. 764-772.

77. Deng, Q.D., et al., Effects of the oral spirochete Treponema denticola on interleukin-8 expression from epithelial cells. Oral Microbiol Immunol, 2001. 16(3): p. 185-7.

78. Jo, A.R., et al., Mechanisms of IL-8 suppression by Treponema denticola in gingival epithelial cells. Immunol Cell Biol, 2014. 92(2): p. 139-47.

79. Hajishengallis, G. and R.J. Lamont, Dancing with the Stars: How Choreographed Bacterial Interactions Dictate Nososymbiocity and Give Rise to Keystone Pathogens, Accessory Pathogens, and Pathobionts. Trends Microbiol, 2016. 24(6): p. 477-489.

80. Mazumdar, V., S. Amar, and D. Segre, Metabolic proximity in the order of colonization of a microbial community. PLoS One, 2013. 8(10): p. e77617.

81. Jorth, P., et al., Metatranscriptomics of the human oral microbiome during health and disease. mBio, 2014. 5(2): p. e01012-14. 
82. Mark Welch, J.L., et al., Biogeography of a human oral microbiome at the micron scale. Proc Natl Acad Sci U S A, 2016. 113(6): p. E791-800.

83. Diaz, P.I., P.S. Zilm, and A.H. Rogers, Fusobacterium nucleatum supports the growth of Porphyromonas gingivalis in oxygenated and carbon-dioxide-depleted environments. Microbiology, 2002. 148(Pt 2): p. 467-472.

84. Palmer, R.J., Jr., et al., Mutualism versus independence: strategies of mixedspecies oral biofilms in vitro using saliva as the sole nutrient source. Infect Immun, 2001. 69(9): p. 5794-804.

85. Kreth, J., et al., The road less traveled - defining molecular commensalism with Streptococcus sanguinis. Mol Oral Microbiol, 2017. 32(3): p. 181-196.

86. Redanz, S., et al., Live and let die: Hydrogen peroxide production by the commensal flora and its role in maintaining a symbiotic microbiome. Mol Oral Microbiol, 2018. 33(5): p. 337-352.

87. Brown, S.A. and M. Whiteley, A novel exclusion mechanism for carbon resource partitioning in Aggregatibacter actinomycetemcomitans. J Bacteriol, 2007. 189(17): p. 6407-14.

88. Duan, D., et al., Fine-tuned production of hydrogen peroxide promotes biofilm formation of Streptococcus parasanguinis by a pathogenic cohabitant Aggregatibacter actinomycetemcomitans. Environ Microbiol, 2016. 18(11): p. 4023-4036.

89. Egland, P.G., R.J. Palmer, Jr., and P.E. Kolenbrander, Interspecies communication in Streptococcus gordonii-Veillonella atypica biofilms: signaling in flow conditions requires juxtaposition. Proc Natl Acad Sci U S A, 2004. 101(48): p. 16917-22.

90. Stacy, A., et al., Bacterial fight-and-flight responses enhance virulence in a polymicrobial infection. Proc Natl Acad Sci U S A, 2014. 111(21): p. 7819-24.

91. Kaplan, J.B., et al., Genes involved in the synthesis and degradation of matrix polysaccharide in Actinobacillus actinomycetemcomitans and Actinobacillus pleuropneumoniae biofilms. J Bacteriol, 2004. 186(24): p. 8213-20.

92. Stacy, A., et al., A Commensal Bacterium Promotes Virulence of an Opportunistic Pathogen via Cross-Respiration. mBio, 2016. 7(3).

93. Hommais, F., et al., Large-scale monitoring of pleiotropic regulation of gene expression by the prokaryotic nucleoid-associated protein, H-NS. Mol Microbiol, 2001. 40(1): p. 20-36.

94. Bao, K., et al., Aggregatibacter actinomycetemcomitans H-NS promotes biofilm formation and alters protein dynamics of other species within a polymicrobial oral biofilm. NPJ Biofilms Microbiomes, 2018. 4: p. 12.

95. Bao, K., et al., Quantitative proteomics reveal distinct protein regulations caused by Aggregatibacter actinomycetemcomitans within subgingival biofilms. PLoS One, 2015. 10(3): p. e0119222.

96. Moye, Z.D., C.M. Gormley, and M.E. Davey, Galactose Impacts the Size and Intracellular Composition of the Asaccharolytic Oral Pathobiont Porphyromonas gingivalis. Appl Environ Microbiol, 2019. 85(4). 
97. Periasamy, S. and P.E. Kolenbrander, Mutualistic biofilm communities develop with Porphyromonas gingivalis and initial, early, and late colonizers of enamel. J Bacteriol, 2009. 191(22): p. 6804-11.

98. Daep, C.A., et al., Structural dissection and in vivo effectiveness of a peptide inhibitor of Porphyromonas gingivalis adherence to Streptococcus gordonii. Infect Immun, 2011. 79(1): p. 67-74.

99. Mahmoud, M.Y., J.M. Steinbach-Rankins, and D.R. Demuth, Functional assessment of peptide-modified PLGA nanoparticles against oral biofilms in a murine model of periodontitis. J Control Release, 2019. 297: p. 3-13.

100. Kuboniwa, M., et al., Streptococcus gordonii utilizes several distinct gene functions to recruit Porphyromonas gingivalis into a mixed community. Mol Microbiol, 2006. 60(1): p. 121-39.

101. Kuboniwa, M., et al., Metabolic crosstalk regulates Porphyromonas gingivalis colonization and virulence during oral polymicrobial infection. Nat Microbiol, 2017. 2(11): p. 1493-1499.

102. Lamont, R.J., H. Koo, and G. Hajishengallis, The oral microbiota: dynamic communities and host interactions. Nat Rev Microbiol, 2018. 16(12): p. 745-759.

103. Cugini, C., et al., Arginine deiminase inhibits Porphyromonas gingivalis surface attachment. Microbiology, 2013. 159(Pt 2): p. 275-285.

104. Ho, M.H., et al., Characterization and development of SAPP as a specific peptidic inhibitor that targets Porphyromonas gingivalis. Mol Oral Microbiol, 2018. 33(6): p. 430-439.

105. Sarkar, J., et al., Transcriptional responses of Treponema denticola to other oral bacterial species. PLoS One, 2014. 9(2): p. e88361.

106. Grenier, D., Nutritional interactions between two suspected periodontopathogens, Treponema denticola and Porphyromonas gingivalis. Infect Immun, 1992. 60(12): p. 5298-301.

107. Tan, K.H., et al., Porphyromonas gingivalis and Treponema denticola exhibit metabolic symbioses. PLoS Pathog, 2014. 10(3): p. e1003955.

108. Martin, B., et al., New growth media for oral bacteria. J Microbiol Methods, 2018. 153: p. 10-13.

109. Deng, Z.L., et al., Worlds Apart - Transcriptome Profiles of Key Oral Microbes in the Periodontal Pocket Compared to Single Laboratory Culture Reflect Synergistic Interactions. Front Microbiol, 2018. 9: p. 124.

110. Nowicki, E.M., et al., Microbiota and Metatranscriptome Changes Accompanying the Onset of Gingivitis. mBio, 2018. 9(2).

111. Cugini, C., et al., Porphyromonas gingivalis: keeping the pathos out of the biont. J Oral Microbiol, 2013. 5.

112. Bornigen, D., et al., Alterations in oral bacterial communities are associated with risk factors for oral and oropharyngeal cancer. Sci Rep, 2017. 7(1): p. 17686.

113. Flynn, K.J., N.T. Baxter, and P.D. Schloss, Metabolic and Community Synergy of Oral Bacteria in Colorectal Cancer. mSphere, 2016. 1(3). 
114. Yost, S., et al., Increased virulence of the oral microbiome in oral squamous cell carcinoma revealed by metatranscriptome analyses. Int J Oral Sci, 2018. 10(4): p. 32.

115. Perera, M., et al., Inflammatory Bacteriome and Oral Squamous Cell Carcinoma. J Dent Res, 2018. 97(6): p. 725-732.

116. Duran-Pinedo, A.E., V.D. Baker, and J. Frias-Lopez, The periodontal pathogen Porphyromonas gingivalis induces expression of transposases and cell death of Streptococcus mitis in a biofilm model. Infect Immun, 2014. 82(8): p. 3374-82.

117. Mukherjee, P.K., et al., Bacteriome and mycobiome associations in oral tongue cancer. Oncotarget, 2017. 8(57): p. 97273-97289.

118. Al-Hebshi, N.N., W.S. Borgnakke, and N.W.J.C.O.H.R. Johnson, The Microbiome of Oral Squamous Cell Carcinomas: a Functional Perspective. 2019. 6(2): p. 145-160.

119. Moffatt-Jauregui, C.E., et al., Establishment and characterization of a telomerase immortalized human gingival epithelial cell line. J Periodontal Res, 2013. 48(6): p. 713-21.

120. Dickman, C.T., et al., Molecular characterization of immortalized normal and dysplastic oral cell lines. J Oral Pathol Med, 2015. 44(5): p. 329-36.

121. Zhou, C.Q., et al., Down-regulation of gamma-synuclein in human esophageal squamous cell carcinoma. World J Gastroenterol, 2003. 9(9): p. 1900-3.

122. Sato, K., et al., Identification of Porphyromonas gingivalis proteins secreted by the Por secretion system. FEMS Microbiol Lett, 2013. 338(1): p. 68-76.

123. Lee, J.Y., et al., Maturation of the Mfa1 Fimbriae in the Oral Pathogen Porphyromonas gingivalis. Front Cell Infect Microbiol, 2018. 8: p. 137.

124. Xie, H., et al., Regulation of the Porphyromonas gingivalis fimA (Fimbrillin) gene. Infect Immun, 2000. 68(12): p. 6574-9.

125. Kuboniwa, M., et al., Distinct roles of long/short fimbriae and gingipains in homotypic biofilm development by Porphyromonas gingivalis. BMC Microbiol, 2009. 9: p. 105.

126. Liu, W., et al., Olfactomedin 4 is a novel target gene of retinoic acids and 5-aza2'-deoxycytidine involved in human myeloid leukemia cell growth, differentiation, and apoptosis. Blood, 2010. 116(23): p. 4938-47.

127. Chastain-Gross, R.P., et al., Genome Sequence of Porphyromonas gingivalis Strain 381. Genome Announc, 2017. 5(2).

128. Chen, T., H. Siddiqui, and I. Olsen, In silico Comparison of 19 Porphyromonas gingivalis Strains in Genomics, Phylogenetics, Phylogenomics and Functional Genomics. Front Cell Infect Microbiol, 2017. 7: p. 28.

129. Dashper, S.G., et al., Porphyromonas gingivalis Uses Specific Domain Rearrangements and Allelic Exchange to Generate Diversity in Surface Virulence Factors. Front Microbiol, 2017. 8: p. 48.

130. To, T.T., et al., Draft Genome Sequence of Low-Passage Clinical Isolate Porphyromonas gingivalis MP4-504. Genome Announc, 2016. 4(2).

131. Lu, J., et al., Endothelial cells promote the colorectal cancer stem cell phenotype through a soluble form of Jagged-1. Cancer Cell, 2013. 23(2): p. 171-85. 
132. Zavadil, J., et al., Integration of TGF-beta/Smad and Jagged1/Notch signalling in epithelial-to-mesenchymal transition. EMBO J, 2004. 23(5): p. 1155-65.

133. Vincent, M.S., et al., Characterization of the Porphyromonas gingivalis Type IX Secretion Trans-envelope PorKLMNP Core Complex. J Biol Chem, 2017. 292(8): p. 3252-3261.

134. Bozkulak, E.C. and G. Weinmaster, Selective use of ADAM10 and ADAM17 in activation of Notch1 signaling. Mol Cell Biol, 2009. 29(21): p. 5679-95.

135. Wu, L., et al., High-throughput protease activity cytometry reveals dosedependent heterogeneity in PMA-mediated ADAM17 activation. Integr Biol (Camb), 2015. 7(5): p. 513-24.

136. Tribble, G.D. and R.J. Lamont, Bacterial invasion of epithelial cells and spreading in periodontal tissue. Periodontol 2000, 2010. 52(1): p. 68-83.

137. Xia, Q., et al., Quantitative proteomics of intracellular Porphyromonas gingivalis. Proteomics, 2007. 7(23): p. 4323-37.

138. Jakubovics, N.S., et al., Role of hydrogen peroxide in competition and cooperation between Streptococcus gordonii and Actinomyces naes/undii. FEMS Microbiol Ecol, 2008. 66(3): p. 637-44.

139. Lamont, R.J., Hydrogen peroxide is a central determinant of oral polymicrobial synergy. Environ Microbiol, 2016. 18(11): p. 3609-3611.

140. Potempa, J. and K.A. Nguyen, Purification and characterization of gingipains. Curr Protoc Protein Sci, 2007. Chapter 21: p. Unit 2120.

141. Liu, X., et al., Real-time mapping of a hydrogen peroxide concentration profile across a polymicrobial bacterial biofilm using scanning electrochemical microscopy. Proc Natl Acad Sci U S A, 2011. 108(7): p. 2668-73.

142. Shin, J.M., et al., Microbial Communities Associated with Primary and Metastatic Head and Neck Squamous Cell Carcinoma - A High Fusobacterial and Low Streptococcal Signature. Sci Rep, 2017. 7(1): p. 9934.

143. Hasegawa, Y., et al., Gingival epithelial cell transcriptional responses to commensal and opportunistic oral microbial species. Infect Immun, 2007. 75(5): p. 2540-7.

144. Mans, J.J., et al., The degree of microbiome complexity influences the epithelial response to infection. BMC Genomics, 2009. 10: p. 380.

145. Ashizawa, Y., et al., OLFM4 Enhances STAT3 Activation and Promotes Tumor Progression by Inhibiting GRIM19 Expression in Human Hepatocellular Carcinoma. Hepatol Commun, 2019. 3(7): p. 954-970.

146. Kreth, J., Y. Zhang, and M.C. Herzberg, Streptococcal antagonism in oral biofilms: Streptococcus sanguinis and Streptococcus gordonii interference with Streptococcus mutans. J Bacteriol, 2008. 190(13): p. 4632-40. 


\title{
1. Personal Information
}

\section{CURRICULUM VITA}

\author{
Name: $\quad$ Zackary Ray Fitzsimonds \\ Work Address: $\quad 570$ S. Preston St. \\ Baxter 1 Room 224 \\ Louisville, KY 40202 \\ Personal Phone: (615) 439- 5981 \\ Email: $\quad$ zrfitz01@louisville.edu
}

\section{Education}
$2016-2023$
D.M.D. / Ph.D. Department of Oral Immunology and Infectious Diseases, University of Louisville School of Dentistry, University of Louisville, Louisville, KY Mentor: Dr. Richard J. Lamont.
2012 - $2015 \quad$ B.S. in Biology (concentration in Physiology), Chemistry minor, College of Basic and Applied Science, Middle Tennessee State University, Murfreesboro, TN

\section{Training and Experience}

During my PhD training I have gained experience in: Cell culturing, isolating and growing primary gingival epithelial cells from whole human tissue, siRNA transfection, plasmid construction, overexpression in epithelial cells, PCR, qRTPCR, SDS-PAGE gels, Western Blots, confocal microscopy, luciferase promoter assays, growth of anaerobic bacteria, genetic manipulation of bacteria, Matrigel invasion assays, protein co-immunoprecipitation, protein-DNA chromatin immunoprecipitation (ChIP), RNAseq, RNAseq pathway analysis, mouse models including 4NQO induced OSCC and Baker's model for periodontitis, classifying mouse tongue biopsies histologically, and mouse euthanasia.

\section{Funding, Awards, and Other Honors}

\subsection{Funding}


2018-2022

Ruth L. Kirschstein Nation Research Service Awards for Individual Predoctoral Fellows, NIDCR, NIH, "Regulation of ZEB1 by Porphyromonas gingivalis in gingival epithelial cells", F30DE028166

\subsection{Awards}

2019

2019

2018

2018

Research

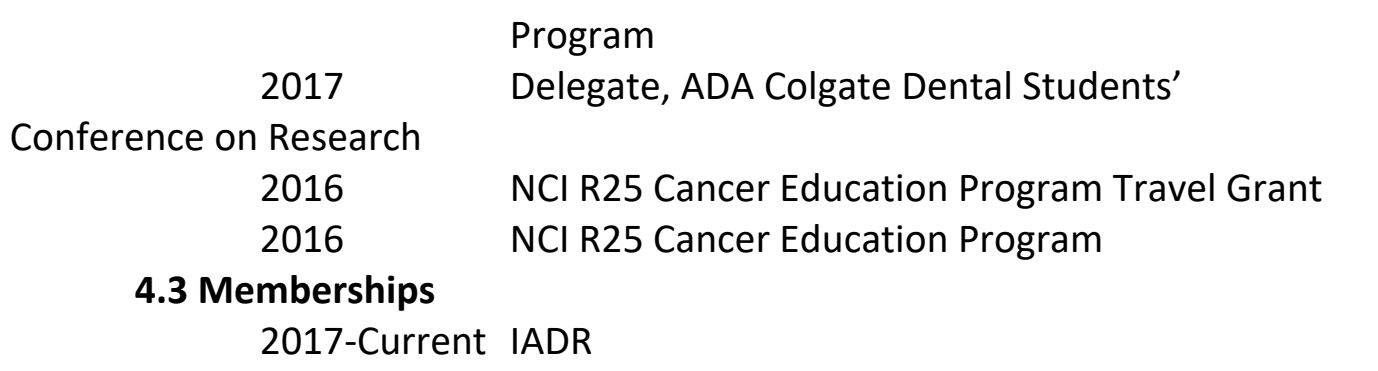

Arnold Bleiweis Travel Award, IADR

AADR Bloc Travel Grant

$2^{\text {nd }}$ Place, Research! Louisville

University of Louisville School of Dentistry Summer

Program

5. Bibliography

\subsection{Manuscripts}

1. Ohshima J*, Wang Q*, Fitzsimonds ZR, Miller DP, Sztukowska MN, Jung YJ, Hayashi M, Whiteley M, Lamont

RJ. Streptococcus gordonii programs epithelial cells to resist ZEB2 induction by Porphyromonas gingivalis. Proc Natl Acad Sci U S A. 2019 Apr 23;116(17):8544-8553.

2. Miller DP, Fitzsimonds ZR, Lamont RJ. Metabolic Signaling and Spatial Interactions in the Oral Polymicrobial Community. J Dent Res. 2019 Nov;98(12):1308-1314.

3. Jung YJ, Miller DP, Perpich JD, Fitzsimonds ZR, Shen D, Ohshima J, Lamont RJ. Porphyromonas gingivalis Tyrosine Phosphatase Php1 Promotes Community Development and Pathogenicity. mBio. 2019 Sep 24;10(5).

4. Fitzsimonds ZR, Rodriguez-Hernandez CJ, Bagaitkar J, Lamont RJ. From Beyond the Pale to the Pale Riders: The Emerging Association of Bacteria with Oral Cancer. J Dent Res. 2020 Jun;99(6):604-612.

\subsection{Poster presentations}

1. Fitzsimonds ZR, Oshima J, Wang Q, Miller DP, Sztukowska M, Jung YJ, Hayashi M, Whiteley M, Lamont RJ. Streptococcus gordonii antagonizes Porphyromonas gingivalis-mediated 
signaling in epithelial cells 2019. Penn Periodontal Conference 2019. Philadelphia, PA. 2019.

2. Fitzsimonds ZR, Oshima J, Lamont RJ. Streptococcus gordonii inhibits Porphyromonas gingivalis induced ZEB2 upregulation in gingival epithelial cells through long non-coding RNA ESRG. Research! Louisville. 2018.

3. Fitzsimonds ZR, Sztukowska M, Metzler M, Darling D, Rouchka E, Kalbfleisch T, Lamont RJ. Differential regulation of long noncoding RNAs in gingival epithelial cells by Porphyromonas gingivalis. IADR. San Francisco, CA. 2017.

\subsection{Oral Presentations}

"Streptococcus gordonii antagonizes Porphyromonas gingivalis-mediated signaling in epithelial cells." IADR. Vancouver, Canada. 2019.

"Streptococcus gordonii antagonizes Porphyromonas gingivalis-mediated signaling in epithelial cells." Penn Periodontology 2019. Philadelphia, PA. 2019.

\section{Mentorship}

Ruth Maclntyre (MS)- University of Louisville School of Dentistry Steven Chapman (MD)- University of Louisville School of Medicine Ankur Patel (BS)- University of Louisville Daonan Shen (MD-PhD)- University of Louisville 